\title{
ON THE TENSOR SEMIGROUP OF AFFINE KAC-MOODY LIE ALGEBRAS
}

\author{
NICOLAS RESSAYRE
}

\begin{abstract}
In this paper, we are interested in the decomposition of the tensor product of two representations of a symmetrizable Kac-Moody Lie algebra $\mathfrak{g}$. Let $P_{+}$be the set of dominant integral weights. For $\lambda \in P_{+}, L(\lambda)$ denotes the irreducible, integrable, highest weight representation of $\mathfrak{g}$ with highest weight $\lambda$. Let $P_{+, \mathbb{Q}}$ be the rational convex cone generated by $P_{+}$. Consider the tensor cone

$\Gamma(\mathfrak{g}):=\left\{\left(\lambda_{1}, \lambda_{2}, \mu\right) \in P_{+, \mathbb{Q}}^{3} \mid \exists N>1 \quad L(N \mu) \subset L\left(N \lambda_{1}\right) \otimes L\left(N \lambda_{2}\right)\right\}$.

If $\mathfrak{g}$ is finite dimensional, $\Gamma(\mathfrak{g})$ is a polyhedral convex cone described in BK06, by an explicit finite list of inequalities. In general, $\Gamma(\mathfrak{g})$ is nor polyhedral, nor closed. In this article we describe the closure of $\Gamma(\mathfrak{g})$ by an explicit countable family of linear inequalities, when $\mathfrak{g}$ is untwisted affine. This solves a Brown-Kumar's conjecture [BK14] in this case.

We also obtain explicit saturation factors for the semigroup of triples $\left(\lambda_{1}, \lambda_{2}, \mu\right) \in P_{+}^{3}$ such that $L(\mu) \subset L\left(\lambda_{1}\right) \otimes L\left(\lambda_{2}\right)$. Note that even the existence of such saturation factors is not obvious since the semigroup is not finitely generated. For example, in case $\tilde{A}_{n}$, we prove that any integer $d_{0} \geq 2$ is a saturation factor, generalizing the case $\tilde{A}_{1}$ shown in [BK14].
\end{abstract}

\section{INTRODUCTION}

Let $A$ be a symmetrizable irreducible GCM of size $l+1$. Let $\mathfrak{h} \supset$ $\left\{\alpha_{0}^{\vee}, \ldots, \alpha_{l}^{\vee}\right\}$ and $\mathfrak{h}^{*} \supset\left\{\alpha_{0}, \ldots, \alpha_{l}\right\}=: \Delta$ be a realization of $A$. We fix an integral form $\mathfrak{h}_{\mathrm{z}} \subset \mathfrak{h}$ containing each $\alpha_{i}^{\vee}$, such that $\mathfrak{h}_{\mathrm{z}}^{*}:=$ $\operatorname{Hom}\left(\mathfrak{h}_{\mathrm{z}}, \mathrm{z}\right)$ contains $\Delta$ and such that $\mathfrak{h}_{\mathrm{z}} / \oplus \mathrm{z} \alpha_{i}^{\vee}$ is torsion free. Set $\mathfrak{h}_{Q}^{*}=\mathfrak{h}_{\mathrm{z}}^{*} \otimes \mathrm{Q} \subset \mathfrak{h}^{*}, P_{+, Q}:=\left\{\lambda \in \mathfrak{h}_{\mathrm{Q}}^{*} \mid\left\langle\alpha_{i}^{\vee}, \lambda\right\rangle \geq 0 \quad \forall i\right\}$, and $P_{+}=$ $\mathfrak{h}_{\mathrm{z}} \cap P_{+, Q}$.

Let $\mathfrak{g}=\mathfrak{g}(A)$ be the associated Kac-Moody Lie algebra with Cartan subalgebra $\mathfrak{h}$. For $\lambda \in P_{+}, L(\lambda)$ denotes the irreducible, integrable, highest weight representation of $\mathfrak{g}$ with highest weight $\lambda$. Define the saturated tensor semigroup as

$\Gamma(A):=\left\{\left(\lambda_{1}, \lambda_{2}, \mu\right) \in P_{+, Q}^{3} \mid \exists N>1 \quad L(N \mu) \subset L\left(N \lambda_{1}\right) \otimes L\left(N \lambda_{2}\right)\right\}$.

In the case that $\mathfrak{g}$ is a semisimple Lie algebra, $\Gamma(A)$ (also denoted by $\Gamma(\mathfrak{g}))$ is a closed convex polyhedral cone given by an explicit set 
of inequalities parametrized by some structure constants of the cohomology rings of the flag varieties for the corresponding algebraic group (see [BK06, Kum14, Kum15, Res10]).

In the general case, $\Gamma(A)$ is no longer closed or polyhedral. Nevertheless, in this paper we describe the closure of $\Gamma(A)$ by infinitely many explicit linear inequalities, if $\mathfrak{g}$ is affine untwisted. Note that some intermediate results are true for any symmetrizable GCM $A$.

Let $G$ be the minimal Kac-Moody group as in [Kum02, Section 7.4] and $B$ its standard Borel subgroup. Fix $\left(\varpi_{\alpha_{0}^{\vee}}, \ldots, \varpi_{\alpha_{l}^{\vee}}\right) \subset \mathfrak{h}_{Q}$ be elements dual to the simple roots. Let $W$ be the Weyl group of $A$. To any simple root $\alpha_{i}$, is associated a maximal standard parabolic subgroup $P_{i}$, its Weyl group $W_{P_{i}} \subset W$ and the set $W^{P_{i}}$ of minimal length representative of elements of $W / W_{P_{i}}$. We also consider the partial flag indvariety $X_{i}=G / P_{i}$ containing the Schubert varieties $X_{w}=\overline{B w P_{i} / P_{i}}$, for $w \in W^{P_{i}}$. Let $\left\{\epsilon_{w}\right\}_{w \in W^{P_{i}}} \subset \mathrm{H}^{*}\left(X_{i}, \mathrm{z}\right)$ be the Schubert basis dual to the basis of the singular homology of $X_{i}$ given by the fundamental classes of $X_{w}$. As defined by Belkale-Kumar [BK06, Section 6] in the finite dimensional case, Brown-Kumar defined in [BK14, Section 7] a deformed product $\odot_{0}$ in $\mathrm{H}^{*}\left(X_{i}, \mathrm{z}\right)$, which is commutative and associative.

Theorem 1. Let $\mathfrak{g}$ ba an affine untwisted Kac-Moody Lie algebra with central element c. Let $\left(\lambda_{1}, \lambda_{2}, \mu\right) \in P_{+, Q}^{3}$ such that $\lambda_{1}(c)>0$ and $\lambda_{2}(c)>0$.

Then,

$$
\left(\lambda_{1}, \lambda_{2}, \mu\right) \in \Gamma(\mathfrak{g})
$$

if and only if

$$
\mu(c)=\lambda_{1}(c)+\lambda_{2}(c),
$$

and

$$
\left\langle\mu, v \varpi_{\alpha_{i}^{\vee}}\right\rangle \leq\left\langle\lambda_{1}, u_{1} \varpi_{\alpha_{i}^{\vee}}\right\rangle+\left\langle\lambda_{2}, u_{2} \varpi_{\alpha_{i}^{\vee}}\right\rangle
$$

for any $i \in\{0, \ldots, l\}$ and any $\left(u_{1}, u_{2}, v\right) \in\left(W^{P_{i}}\right)^{3}$ such that $\epsilon_{v}$ occurs with coefficient 1 in the deformed product

$$
\epsilon_{u_{1}} \odot_{0} \epsilon_{u_{2}}
$$

The statement of Theorem 1 is very similar to [BK06, Theorem 22] that describes $\Gamma(\mathfrak{g})$, if $\mathfrak{g}$ is finite dimensional. Nevertheless, the proof is very different. Indeed, in the classical case the main ingredients are Kempf's semistability theory and Hilbert-Mumford's theorem (see [BK06] or [Res10]). These results have no known generalization in our situation. We overcome this difficulty by using a new strategy that we now explain roughly speaking. 
Consider the cone $\mathcal{C}(\mathfrak{g})$ defined by equality (1) and inequalities (2). It remains to prove that, up to the assumption " $\lambda_{1}(c)$ and $\lambda_{2}(c)$ are positive", the cone $\mathcal{C}(\mathfrak{g})$ is equal to $\Gamma(\mathfrak{g})$. The proof proceeds in fives steps.

STEP 1. $\Gamma(\mathfrak{g})$ is convex.

This is a well-known consequence of Borel-Weil's theorem (see Lemma 4).

STEP 2. The set $\Gamma(\mathfrak{g})$ is contained in $\mathcal{C}(\mathfrak{g})$.

This step is proved in BK14 and reproved here. The first ingredient is the easy implication in the Hilbert-Mumford's theorem. Indeed "semistable $\Rightarrow$ numerically semistable" is still true for ind-varieties and ind-groups. In the finite dimensional case, the second argument is Kmeiman's transversality theorem. In BK14, this is by an argument in K-theory which express the structure constants of $H^{*}\left(G / P_{i}\right.$, z $)$ as the Euler characteristic of sheaves supported by the intersection of three translated Schubert or Birkhoff varieties. Here, we refine this argument by proving a version of Kleiman's theorem that allows to express these structure constants as the cardinality of three translated Schubert or Birkhoff varieties.

STEP 3. The cone $\mathcal{C}(\mathfrak{g})$ is locally polyhedral.

This is a consequence of Proposition 4 below. We study the inequalities (2) defining $\mathcal{C}(\mathfrak{g})$. In particular, we use some consequences of the nonvanishing of a structure constant of the ring $H^{*}(G / P, \mathrm{z}$ ) (see Lemmas 17 and 18 below or [BK14]).

STEP 4. Study of the boundary of $\mathcal{C}(\mathfrak{g})$.

Let $\left(\lambda_{1}, \lambda_{2}, \mu\right)$ be an integral point in the boundary of $\mathcal{C}(\mathfrak{g})$. Step 3 implies that some inequality (2) has to be an equality for $\left(\lambda_{1}, \lambda_{2}, \mu\right)$. Then, one can use the following Theorem 2 to describe inductively the multiplicity of $L(\mu)$ in $L\left(\lambda_{1}\right) \otimes L\left(\lambda_{2}\right)$. Let $\alpha_{i}$ be a simple root and let $L_{i}$ denote the standard Levi subgroup of $P_{i}$. For $w \in W^{P_{i}}$ and $\lambda \in P_{+}, w^{-1} \lambda$ is a dominant weight for $L_{i}$ : we denote by $L_{L_{i}}\left(w^{-1} \lambda\right)$ the corresponding irreducible highest weight $L_{i}$-module.

Theorem 2. Here, $\mathfrak{g}$ is any symmetrizable Kac-Moody Lie algebra and $\alpha_{i}$ is a simple root. Let $\left(\lambda_{1}, \lambda_{2}, \mu\right) \in P_{+}^{3}$. Let $\left(u_{1}, u_{2}, v\right) \in\left(W^{P_{i}}\right)^{3}$ such that $\epsilon_{v}$ occurs with coefficient 1 in the ordinary product $\epsilon_{u_{1}} . \epsilon_{u_{2}}$. We assume that

$$
\left\langle\mu, v \varpi_{\alpha_{i}^{\vee}}\right\rangle=\left\langle\lambda_{1}, u_{1} \varpi_{\alpha_{i}^{\vee}}\right\rangle+\left\langle\lambda_{2}, u_{2} \varpi_{\alpha_{i}^{\vee}}\right\rangle .
$$

Then the multiplicity of $L(\mu)$ in $L\left(\lambda_{1}\right) \otimes L\left(\lambda_{2}\right)$ is equal to the multiplicity of $L_{L_{i}}\left(v^{-1} \mu\right)$ in $L_{L_{i}}\left(u_{1}^{-1} \lambda_{1}\right) \otimes L_{L_{i}}\left(u_{2}^{-1} \lambda_{2}\right)$

Note that Theorem 5 and its corollary in Section 6 are a little bit stronger than Theorem 2 , 
STEP 5. Induction.

Whereas there are numerous technical difficulties the basic idea is simple. By convexity, it is sufficient to prove that the boundary of $\mathcal{C}(\mathfrak{g})$ is contained in $\Gamma(\mathfrak{g})$. Using Step 4, this can be proved by induction.

More precisely, consider a face $\mathcal{F}$ of codimension one of $\mathcal{C}(\mathfrak{g})$ associated to some structure constant of $H^{*}\left(G / P_{i}, \mathrm{z}\right)$ for $\odot_{0}$ equal to one. We have to prove that $\mathcal{F}$ is contained in $\Gamma(\mathfrak{g})$. By Theorem 2 , it remains to prove that the points of $\mathcal{F}$ satisfy the inequalities that characterize $\Gamma\left(L_{i}\right)$. Fix such an inequality associated to a structure constant of $H^{*}\left(L_{i} /\left(P_{j} \cap L_{i}\right), \mathrm{z}\right)$ for $\odot_{0}$ equal to one. Consider the flags ind-varieties:

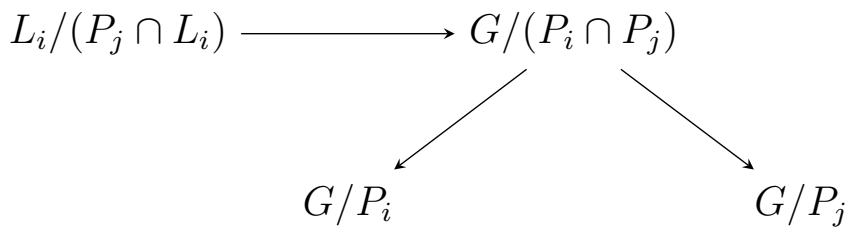

Proposition 3 that shows a property of multiplicativity for structure constants of the rings $H^{*}(G / P, \mathrm{z})$ gives us a structure constant of $H^{*}\left(G /\left(P_{i} \cap P_{j}\right), \mathrm{z}\right)$ equal to one, for the ordinary product. An important point is Theorem 6 that proves that, if the considered inequality of $\Gamma\left(L_{i}\right)$ is "useful" then this structure constant of $H^{*}\left(G /\left(P_{i} \cap P_{j}\right)\right.$, z) is actually nonzero for $\odot_{0}$. Then it gives a structure constant of $H^{*}\left(G /\left(P_{j}\right), \mathrm{z}\right)$ for $\odot_{0}$ equal to one. In particular, this gives an inequality of $\mathcal{C}(\mathfrak{g})$ and the inequality we wanted to prove for the points of $\mathcal{F}$.

If we prove Theorem 1 only for the untwisted affine case, the general strategy should works more generally. For this reason, we prove some intermediate results for any symmetrizable Kac-Moody Lie algebra. In particular Steps 1, 2 and 4 works with this generality. Proposition 3 of multiplicativity also holds in this context.

Let $Q$ denote the root lattice of $\mathfrak{g}$. Consider the tensor semigroup

$$
\Gamma_{\mathrm{N}}(\mathfrak{g}):=\left\{\left(\lambda_{1}, \lambda_{2}, \mu\right) \in P_{+}^{3} \mid \exists N>1 \quad L(\mu) \subset L\left(\lambda_{1}\right) \otimes L\left(\lambda_{2}\right)\right\} .
$$

It is actually a semigroup but it is not finitely generated for $\mathfrak{g}$ affine. Despite this, we obtain explicit saturation factors: a positive integer $d_{0}$ is called a saturation factor for $\mathfrak{g}$ if for any $\left(\lambda_{1}, \lambda_{2}, \mu\right) \in \Gamma(\mathfrak{g}) \cap\left(P_{+}\right)^{3}$ such that $\lambda_{1}+\lambda_{2}-\mu \in Q, L\left(d_{0} \mu\right)$ is a submodule of $L\left(d_{0} \lambda_{1}\right) \otimes L\left(d_{0} \lambda_{2}\right)$. Observe that the condition $\lambda_{1}+\lambda_{2}-\mu \in Q$ is necessary to have $L(\mu) \subset$ $L\left(\lambda_{1}\right) \otimes L\left(\lambda_{2}\right)$.

To describe our saturation factors, we need additional notation. Up to now, $\mathfrak{g}$ is the affine Lie algebra associated to the simple Lie algebra $\dot{\mathfrak{g}}$. Let us define the constant $k_{s}$ to be the least common multiple of saturation factors of maximal Levi subalgebras of $\mathfrak{g}$. The value of 
$k_{s}$ depends on known saturation factors for the finte dimensional Lie algebras. With the actual literature (see Section 10), possible values for $k_{s}$ are given in the following tabular.

\begin{tabular}{|c|c|c|c|c|c|c|}
\hline Type of $\dot{\mathfrak{g}}$ & $A_{\ell}$ & $B_{3} B_{4}$ & $B_{\ell}(\ell \geq 5)$ & $C_{\ell}(\ell \geq 2)$ & $D_{4}$ & $D_{\ell}(\ell \geq 5)$ \\
\hline$k_{s}$ & 1 & 2 & 4 & 2 & 1 & 4 \\
\hline \hline Type of $\dot{\mathfrak{g}}$ & $E_{6}$ & $E_{7}$ & $E_{8}$ & $F_{4}$ & $G_{2}$ & $G_{2}$ \\
\hline$k_{s}$ & 36 & 144 & 3600 & 144 & 2 & 3 \\
\hline
\end{tabular}

Let $k_{\mathfrak{g}}$ be the least common multiple of coordinates of $\dot{\theta}$ written in terms of the simple roots. The values of $k_{\mathfrak{g}}$ are

\begin{tabular}{|c|c|c|c|c|c|c|c|c|c|}
\hline Type & $A_{\ell}$ & $B_{\ell}(\ell \geq 2)$ & $C_{\ell}(\ell \geq 3)$ & $D_{\ell}(\ell \geq 5)$ & $E_{6}$ & $E_{7}$ & $E_{8}$ & $F_{4}$ & $G_{2}$ \\
\hline$k_{\mathfrak{g}}$ & 1 & 2 & 2 & 2 & 6 & 12 & 60 & 12 & 6 \\
\hline
\end{tabular}

Theorem 3. Let $\left(\lambda_{1}, \lambda_{2}, \mu\right) \in\left(P_{+}\right)^{3}$ such that there exists $N>0$ such that $L(N \mu)$ embeds in $L\left(N \lambda_{1}\right) \otimes L\left(N \lambda_{2}\right)$. We also assume that $\mu-\lambda_{1}-\lambda_{2} \in Q$.

Then,

(i) if $k_{s}=1$ then any integer $d \geq 2, L\left(d k_{\mathfrak{g}} \mu\right)$ embeds in $L\left(d k_{\dot{\mathfrak{g}}} \lambda_{1}\right) \otimes$ $L\left(d k_{\mathfrak{g}} \lambda_{2}\right)$

(ii) if $k_{s}>1$ then $L\left(k_{\mathfrak{g}} k_{s} \mu\right)$ embeds in $L\left(k_{\mathfrak{g}} k_{s} \lambda_{1}\right) \otimes L\left(k_{\mathfrak{g}} k_{s} \lambda_{2}\right)$.

Observe that, in type $A, k_{\mathfrak{g}} k_{s}=1$. The case $A_{1}$ was obtained before in [BK14].

Let $\delta$ denote the fundamental imaginary root. We also obtain the following variation.

Theorem 4. Let $\left(\lambda_{1}, \lambda_{2}, \mu\right) \in\left(P_{+}\right)^{3}$ such that there exists $N>0$ such that $L(N \mu)$ embeds in $L\left(N \lambda_{1}\right) \otimes L\left(N \lambda_{2}\right)$. We also assume that $\mu-\lambda_{1}-\lambda_{2} \in Q$.

Then, for any integer $d \geq 2, L\left(k_{\mathfrak{g}} k_{s} \mu-d \delta\right)$ embeds in $L\left(k_{\mathfrak{g}} k_{s} \lambda_{1}\right) \otimes$ $L\left(k_{\mathfrak{g}} k_{s} \lambda_{2}\right)$.

In Section 11, we collect some technical lemmas used in the paper.

Acknowledgements. I am pleased to thank Michael Bulois, Stéphane Gaussent, Philippe Gille, Kenji Iohara, Nicolas Perrin, Bertrand Remy for useful discussions.

The author is partially supported by the French National Agency (Project GeoLie ANR-15-CE40-0012).

\section{Contents}

1. Introduction

2. Ind-varieties

2.1. Ind-varieties 
2.2. Irreducibility

2.3. Line bundles

3. Using Borel-Weil Theorem

3.1. Tensor multiplicities

3.2. Multiplicities as dimensions

4. Enumerative meaning of structure constants of $H^{*}(G / P, \mathrm{z})$

4.1. Richardson varieties

4.2. Kleiman's lemma

4.3. The case $n_{u_{1} u_{2}}^{v}=1$

5. Inequalities for $\Gamma(A)$

6. Multiplicities on the boundary

7. The Belkale-Kumar-Brown product

7.1. Preliminaries of linear algebra

7.2. Definition of the BKB product

7.3. On Levi movability

8. Multiplicativity in cohomology

8.1. The multiplicativity

8.2. Application to the BKB-product

9. The untwisted affine case

9.1. Notation

9.2. Essential inequalities and BKB-product

9.3. About $\Gamma(\mathfrak{g})$

9.4. A cone defined by inequalities

9.5. Realisation of $\mathcal{C}$ as an hypograph

9.6. The convex set $\mathcal{C}$ is locally polyhedral

9.7. An example of a face of codimension 1

9.8. The main result

10. Saturation factors

11. Some technical lemmas

11.1. Bruhat and Birkhoff decompositions

11.2. Affine root systems

11.3. Jacobson-Morozov's theorem

11.4. Geometric Invariant Theory

References

\section{IND-VARIETIES}

2.1. Ind-varieties. In this section, we collect definitions, notation and properties of ind-varieties. The results are certainly well-known, but we include some proofs for the convenience of the reader.

2.1.1. The category. Let $\left(X_{n}\right)_{n \in \mathrm{N}}$ be a sequence of quasiprojective complex varieties given with closed immersions $\iota_{n}: X_{n} \rightarrow X_{n+1}$. Consider the inductive limit $X=\lim _{\longrightarrow} X_{n}$. A subset $F$ in $X$ is said to be 
Zariski closed if $F \cap X_{n}$ is closed for any $n \in \mathrm{N}$. A continuous map $f: X \longrightarrow Y=\lim _{n} Y_{n}$ between two ind-varieties is a morphism if for any $n \in \mathrm{N}$ there exists $m \in \mathrm{N}$ such that $f\left(X_{n}\right) \subset Y_{m}$ and the restriction $f: X_{n} \longrightarrow Y_{m}$ is a morphism. Let $X_{n}^{\prime} \subset X$ be closed subsets such that $X=\cup_{n \in \mathbb{N}} X_{n}^{\prime}$ and $X_{0}^{\prime} \subset X_{1}^{\prime} \subset \cdots X_{n}^{\prime} \subset \cdots$. Then $X^{\prime}=\lim _{\longrightarrow} X_{n}^{\prime}$ is an ind-variety. The filtrations $\left(X_{n}\right)_{n \in \mathrm{N}}$ and $\left(X_{n}^{\prime}\right)_{n \in \mathrm{N}}$ are said to be equivalent if the identity maps $X \longrightarrow X^{\prime}$ and $X^{\prime} \longrightarrow X$ are morphisms. Actually, the filtrations on ind-varieties are regarded up to equivalence; an ind-variety $X$ endowed with a filtration $X_{0} \subset X_{1} \subset \cdots X_{n} \subset \cdots$ by closed subsets is called a filtered ind-variety and simply denoted by $X=\cup_{n \in \mathrm{N}} X_{n}$.

Lemma 1. Let $X=\cup_{n \in \mathrm{N}} X_{n}$ be a filtered ind-variety. Assume, we have a family $\left(X_{n}^{\prime}\right)_{n \in \mathrm{N}}$ of closed subsets in $X$ such that $X_{n}^{\prime} \subset X_{n+1}^{\prime}$ and $X=\cup_{n \in \mathrm{N}} X_{n}^{\prime}$.

Then the two filtrations $\left(X_{n}\right)_{n \in \mathbb{N}}$ and $\left(X_{n}^{\prime}\right)_{n \in \mathbb{N}}$ are equivalent.

Proof. Consider an irreducible component $C$ of some $X_{n_{0}}$. Then $C=$ $\cup_{n} X_{n}^{\prime} \cap C$ and $X_{n}^{\prime} \cap C$ is closed in $C$. Assume that for any $n, X_{n}^{\prime} \cap$ $C \neq C$. Then, for any $n, \operatorname{dim}\left(X_{n}^{\prime} \cap C\right)<\operatorname{dim} C$. Hence $C$ is the union of countably many subvarieties of smaller dimension. This is a contradiction since we are working on the uncountable field of complex numbers: there exists $n_{C}$ such that $X_{n_{C}}^{\prime} \cap C=C$.

Since $X_{n_{0}}$ has finitely many irreducible components, there exists $N_{0}$ such that $X_{n_{0}} \subset X_{N_{0}}^{\prime}$.

Observe that $X_{n} \cap X_{m}^{\prime}$ is closed in $X$ and hence in $X_{m}^{\prime}$. Then the same proof as above shows that for any $n_{1}$, there exists $N_{1}$ such that $X_{n_{1}}^{\prime} \subset X_{N_{1}}$.

For $x \in X$, we denote by $T_{x} X$ the tangent space of $X$ at $x$. By definition $T_{x} X=\stackrel{\lim }{\longrightarrow} T_{x} X_{n}$.

2.2. Irreducibility. An ind-variety $X$ is said to be irreducible if it is as a topological space. A poset is said to be directed if for any two elements $x, y$ there exists $z$ bigger or equal to $x$ and $y$. If the poset of irreducible components of the $X_{n}$ 's is directed for inclusion then $X$ is irreducible. Unless [Sha81, Proposition 1], the converse of this assertion is not true (see [Kam96, Sta12 for examples). Here, the ind-variety $X$ is said to be ind-irreducible if the poset of irreducible components of the $X_{n}$ 's is directed for inclusion. The following lemma gives a more precise definition.

Lemma 2. Let $X$ be an ind-variety. The following are equivalent:

(i) there exists a filtration $X=\cup_{n \in \mathrm{N}} X_{n}$ such that the poset of irreducible components of the $X_{n}$ 's is directed; 
(ii) for any equivalent filtration $X=\cup_{n \in \mathbb{N}} X_{n}$, the poset of irreducible components of the $X_{n}$ is directed;

(iii) there exists an equivalent filtration $X=\cup_{n \in \mathrm{N}} X_{n}$ with $X_{n}$ irreducible, for any $n$.

If $X$ satisfies these properties then $X$ is said to be ind-irreducible.

Proof. We prove $(i i) \Rightarrow(i) \Rightarrow(i i i) \Rightarrow(i i)$. The first implication is tautological.

Show $(i) \Rightarrow($ iii $)$. Since $X_{n}$ has finitely many irreducible components the assumption implies that

$\forall n \quad \exists N$ and an irreducible component $C_{N}$ of $X_{N}$ such that $X_{n} \subset C_{N}$.

Then one can construct by induction an increasing sequence $\varphi: \mathrm{N} \longrightarrow$ N and irreducible components $C_{\varphi(n)}$ of $X_{\varphi(n)}$ such that

$$
\forall n \quad X_{\varphi(n)} \subset C_{\varphi(n+1)} \subset X_{\varphi(n+1)} .
$$

Note that the $C_{\varphi(n)}$ 's are closed, satisfy $C_{\varphi(n)} \subset C_{\varphi(n+1)}$ and $X=$ $\cup_{n \in \mathrm{N}} C_{\varphi(n)}$. Moreover, by (41), the filtrations by $X_{n}$ 's and $C_{\varphi(n)}$ 's are equivalent.

Show $(i i i) \Rightarrow\left(\right.$ ii). Fix a filtration $X=\cup_{n \in \mathbb{N}} X_{n}$ with irreducible closed subsets $X_{n}$. Let $X=\cup_{n \in \mathrm{N}} X_{n}^{\prime}$ be an equivalent filtration. Consider two irreducible components $C_{n_{1}}$ and $C_{n_{2}}$ of some $X_{n_{1}}^{\prime}$ and $X_{n_{2}}^{\prime}$. There exist $N_{1}$ and $N_{2}$ in $\mathrm{N}$ such that $C_{n_{1}} \cap C_{n_{2}} \subset X_{N_{1}} \subset X_{N_{2}}^{\prime}$. Since $X_{N_{1}}$ is irreducible, there exists an irreducible component $C_{N_{2}}^{\prime}$ of $X_{N_{2}}^{\prime}$ containing $X_{N_{1}}$. Hence the poset of irreductible components of the $X_{n}^{\prime}$ is directed.

\section{Examples.}

(i) The simplest examples $\mathrm{A}^{(\infty)}=\cup_{n \in \mathrm{N}} \mathrm{A}^{n}, \mathrm{P}^{(\infty)} \cup_{n \in \mathrm{N}} \mathrm{P}^{n}$ of indvarieties are ind-irreducible.

(ii) A nonempty open subset of an ind-irreducible ind-variety is ind-irreducible. A product of two ind-irreducible ind-varieties is ind-irreducible.

(iii) Consider a surjective morphism $f: X \longrightarrow Y$ of ind-varieties. If $X$ is ind-irreducible then so is $Y$. Indeed, let $X_{n}$ be a filtration of $X$ by irreducible subvarieties. Denote by $Y_{n}$ the closure in $Y$ of $f\left(X_{n}\right)$. Then, by Lemma 1, $Y=\cup_{n} Y_{n}$ is an equivalent filtration of $Y$ by irreducible subvarieties. Hence $Y$ is ind-irreducible.

(iv) If $G$ is a Kac-Moody group and $P$ a standard parabolic subgroup then $G / P$ is a projective ind-variety. Indeed, a filtration of $G / P$ is given by the unions of Schubert varieties of bounded dimension. Since the Bruhat order is directed (see e.g. BB05, Proposition 2.2.9]), $G / P$ is ind-irreducible.

(v) The Birkhoff subvarieties of $G / P$ are ind-varieties. Indeed the Richardson varieties are irreducible. 
(vi) Let $G$ be the minimal Kac-Moody group as defined in Kum02, Section 7.4]. Then $G$ is an ind-variety ind-irreducible. Indeed, for each real root $\beta$, denote by $U_{\beta}: \mathrm{C} \longrightarrow G$ the radicial subgroup. Consider an infinite word $\underline{w}=\beta_{1}, \ldots, \beta_{n}, \ldots$ in the real roots of $\mathfrak{g}$ such that any finite word in these roots is a subword of $\underline{w}$. Consider the map

$$
\begin{aligned}
\theta: \mathrm{A}^{(\infty)} \times T & \longrightarrow G \\
\left(\left(\tau_{i}\right)_{i \in \mathrm{N}}, t\right) & \longmapsto\left(\prod_{i} U_{\beta_{i}}\left(\tau_{i}\right)\right) t
\end{aligned}
$$

Since $G$ is an ind-group and $U_{\beta}$ are morphism of ind-groups, $\theta$ is a morphism of ind-varieties. By definition, it is surjective. By Example (iii), $G$ is ind-irreducible.

Another result we need, is the following.

Lemma 3. Let $X$ be an ind-variety ind-irreducible. Let $\Omega$ be a nonempty open subset of $X$.

Let $\left(Y_{n}\right)_{n \in \mathbb{N}}$ be a collection of closed subsets of $X$ such that $Y_{n} \subset Y_{n+1}$ and $\cup_{n \in \mathrm{N}} Y_{n}$ contains $\Omega$.

Then $X=\cup_{n \in \mathrm{N}} Y_{n}$ is an equivalent filtration of $X$.

Proof. Fix a filtration $X=\cup_{n \in \mathrm{N}} X_{n}$ by closed irreducible subsets of $X$ intersecting $\Omega$.

Fix $n_{0} \in \mathrm{N}$. Observe that $X_{n_{0}} \cap \Omega \subset \cup_{n \in \mathrm{N}}\left(X_{n_{0}} \cap Y_{n} \cap \Omega\right)$. But $X_{n_{0}} \cap Y_{n} \cap \Omega$ is a locally closed subvariety of $X_{n_{0}}$. Consider the sequence $n \mapsto \operatorname{dim}\left(X_{n_{0}} \cap Y_{n} \cap \Omega\right)$. It is nondeacreasing.

Assume that $\operatorname{dim}\left(X_{n_{0}} \cap Y_{n} \cap \Omega\right)<\operatorname{dim}\left(X_{n_{0}} \cap \Omega\right)$, for any $n$. Then $X_{n_{0}} \cap \Omega$ is the union of contable many strict subvarieties. This is a contration since the base field is uncountable. Hence there exists $N$ such that $\operatorname{dim}\left(X_{n_{0}} \cap Y_{N} \cap \Omega\right)=\operatorname{dim}\left(X_{n_{0}} \cap \Omega\right)$.

Then, $X_{n_{0}} \cap \Omega$ being irreducible, it is contained in $Y_{N}$. Since $Y_{N}$ is closed, it follows that $X_{n_{0}}$ is contained in $Y_{N}$. In particular, $X=\cup_{n} Y_{n}$. We conclude using Lemma 1 .

2.3. Line bundles. Let $X=\cup_{n \in \mathrm{N}} X_{n}$ be an ind-variety. Denote by $\iota_{n}: X_{n} \longrightarrow X$ the inclusion. A line bundle $\mathcal{L}$ over $X$ is an ind-variety with a morphism $\pi: \mathcal{L} \longrightarrow X$ such that $\iota_{n}^{*}(\mathcal{L})$ is a line bundle over $X_{n}$, for any $n$.

A section of $\mathcal{L}$ is a morphism $\sigma: X \longrightarrow \mathcal{L}$ such that $\pi \circ \sigma=\operatorname{Id}_{X}$. We denote by $H^{0}(X, \mathcal{L})$ the vector space of sections. Given a section $\sigma$, we consider the sequence of sections $\left(\sigma_{n}=\iota_{n}^{*}(\sigma)\right)_{n \in \mathbb{N}}$. Then

$$
\sigma_{n+1 \mid X_{n}}=\sigma_{n}
$$

Conversely, a sequence $\sigma_{n}$ of sections of $\iota_{n}^{*}(\mathcal{L})$ on $X_{n}$ satisfying condition (5) induces a well defined section $\sigma$ of $\mathcal{L}$. 


\section{Using Borel-Weil TheOrem}

3.1. Tensor multiplicities. Recall that $\mathfrak{g}$ is a symmetrizable KacMoody Lie algebra. For given $\lambda_{1}$ and $\lambda_{2}$ in $P_{+}, L\left(\lambda_{1}\right) \otimes L\left(\lambda_{2}\right)$ decomposes as a sum of integrable irreducible highest weights modules (see [Kum02, Corrolary 2.2.7]), with finite multiplicities:

$$
L\left(\lambda_{1}\right) \otimes L\left(\lambda_{2}\right)=\oplus_{\mu \in P_{+}} L(\mu)^{\oplus c_{\lambda_{1} \lambda_{2}}^{\mu}}
$$

Let $M$ be a $\mathfrak{g}$-module in the category $\mathcal{O}$; under the action of $\mathfrak{h}, M$ decomposes as $\oplus_{\mu} M_{\mu}$ with finite dimensional weight spaces $M_{\mu}$. Set $M^{\vee}=\oplus_{\mu} M_{\mu}^{*}$ : it is a sub- $\mathfrak{g}$-module of the dual space $M^{*}$.

3.2. Multiplicities as dimensions. Recall that $G$ is the minimal Kac-Moody group for a given irreducible symmetrizable GCM $A$. Let $B$ be the standard Borel subgroup of $G$ and $B^{-}$be the opposite Borel subgroup. Consider $G / B$ and $G / B^{-}$endowed with the usual ind-variety structures. Let $\underline{o}=B / B$ (resp. $\underline{o}^{-}=B^{-} / B^{-}$) denote the base point of $G / B$ (resp. $\left.G / B^{-}\right)$. For $\lambda \in \mathfrak{h}_{\mathrm{z}}^{*}=\operatorname{Hom}\left(T, \mathrm{C}^{*}\right)=\operatorname{Hom}\left(B, \mathrm{C}^{*}\right)=$ $\operatorname{Hom}\left(B^{-}, C^{*}\right)$, we consider the $G$-linearized line bundle $\mathcal{L}(\lambda)$ (resp. $\left.\mathcal{L}_{-}(\lambda)\right)$ on $G / B$ (resp. $G / B^{-}$) such that $B$ (resp. $B^{-}$) acts on the fiber over $\underline{o}$ (resp. $\left.\underline{o}^{-}\right)$with weight $-\lambda$ (resp. $\lambda$ ). For $\lambda \in P_{+}$, we have $G$-equivariant isomorphisms (see [Kum02, Section VIII.3])

$$
\begin{aligned}
& \mathrm{H}^{0}(G / B, \mathcal{L}(\lambda)) \\
& \mathrm{H}^{0}\left(G / B^{-}, \mathcal{L}_{-}(\lambda)\right) \simeq \operatorname{Hom}(L(\lambda), \mathrm{C}), \\
&
\end{aligned}
$$

Set

$$
\mathrm{x}=\left(G / B^{-}\right)^{2} \times G / B .
$$

A significant part of the following lemma is contained in BK14, Proof of Theorem 3.2].

Lemma 4. Let $\lambda_{1}, \lambda_{2}$, and $\mu$ in $P_{+}$. Then the space

$$
\mathrm{H}^{0}\left(\mathrm{X}, \mathcal{L}_{-}\left(\lambda_{1}\right) \otimes \mathcal{L}_{-}\left(\lambda_{2}\right) \otimes \mathcal{L}(\mu)\right)^{G}
$$

of $G$-invariant sections has dimension $c_{\lambda_{1} \lambda_{2}}^{\mu}$. In particular, this dimension is finite.

Proof. Set $\mathcal{L}=\mathcal{L}_{-}\left(\lambda_{1}\right) \otimes \mathcal{L}_{-}\left(\lambda_{2}\right) \otimes \mathcal{L}(\mu)$. We have the following canonical isomorphisms:

$$
\begin{aligned}
\mathrm{H}^{0}(\mathrm{x}, \mathcal{L})^{G} & \simeq \operatorname{Hom}\left(L\left(\lambda_{1}\right)^{\vee} \otimes L\left(\lambda_{2}\right)^{\vee} \otimes L(\mu), \mathrm{C}\right)^{G} \\
& \simeq \operatorname{Hom}\left(L(\mu),\left(L\left(\lambda_{1}\right)^{\vee} \otimes L\left(\lambda_{2}\right)^{\vee}\right)^{*}\right)^{G} \\
& \simeq \operatorname{Hom}\left(L(\mu),\left(L\left(\lambda_{1}\right)^{\vee} \otimes L\left(\lambda_{2}\right)^{\vee}\right)^{\vee}\right)^{G} \quad \text { by h-invariance } \\
& \simeq \operatorname{Hom}\left(L(\mu), L\left(\lambda_{1}\right) \otimes L\left(\lambda_{2}\right)\right)^{G}
\end{aligned}
$$

Thus this space of invariant sections has dimension $c_{\lambda_{1} \lambda_{2}}^{\mu}$. We already mentioned that $c_{\lambda_{1} \lambda_{2}}^{\mu}$ is finite. Nevertheless, we prove independently that $\mathrm{H}^{0}(\mathrm{X}, \mathcal{L})^{G}$ is finite dimensional, reproving that $c_{\lambda_{1} \lambda_{2}}^{\mu}$ is finite. 
Consider the $T$-equivariant map $\iota: G / B^{-} \longrightarrow \mathrm{X}, x \longmapsto\left(\underline{o}^{-}, x, \underline{o}\right)$. Then $\iota^{*}(\mathcal{L})$ is a $T$-linearized line bundle on $G / B^{-}$. Consider

$$
\iota^{*}: \mathrm{H}^{0}(\mathrm{X}, \mathcal{L}) \longrightarrow \mathrm{H}^{0}\left(G / B^{-}, \iota^{*}(\mathcal{L})\right) .
$$

Since $G$. $\left(\underline{o}^{-}, \underline{o}\right)$ is dense in $G / B^{-} \times G / B$, the restriction of $\iota^{*}$ to $\mathrm{H}^{0}(\mathrm{x}, \mathcal{L})^{G}$ is injective. Since $\iota$ is $T$-equivariant, $\iota^{*}\left(\mathrm{H}^{0}(\mathrm{x}, \mathcal{L})^{G}\right)$ is contained in $\mathrm{H}^{0}\left(G / B^{-}, \iota^{*}(\mathcal{L})\right)^{T}$. But $\iota^{*}(\mathcal{L}) \simeq \mathcal{L}_{-}\left(\lambda_{2}\right) \otimes\left(\lambda_{1}-\mu\right)$. Then

$$
\begin{aligned}
\mathrm{H}^{0}\left(G / B^{-}, \iota^{*}(\mathcal{L})\right)^{T} & \simeq \mathrm{H}^{0}\left(G / B^{-}, \mathcal{L}_{-}\left(\lambda_{2}\right)\right)^{(T)_{\mu-\lambda_{1}}} \\
& \simeq \operatorname{Hom}\left(L\left(\lambda_{2}\right)^{\vee}, \mathrm{C}\right)^{(T)_{\mu-\lambda_{1}}} \\
& \simeq L\left(\lambda_{2}\right)^{(T)_{\mu-\lambda_{1}}}
\end{aligned}
$$

Since $L\left(\lambda_{2}\right)$ belongs to the category $\mathcal{O}$, the dimension of $L\left(\lambda_{2}\right)^{(T)_{\mu-\lambda_{1}}}$ is finite. It follows that $\iota^{*}$ embeds $\mathrm{H}^{0}(\mathrm{X}, \mathcal{L})^{G}$ in a finite dimensional vector space.

\section{Enumerative meaning of StRucture Constants of $H^{*}(G / P, \mathrm{z})$}

4.1. Richardson varieties. Let $U$ be the usual ind-subgroup of $B$. Let $P$ be a standard parabolic subgroup of $G$ and $X=G / P$ be the flag ind-variety.

For $u, v \in W^{P}$, set $X_{G / P}^{u}=\overline{B^{-} u P / P}$ and $X_{v}^{G / P}=\overline{B v P / P}$. Set also $\stackrel{\circ}{X}_{G / P}^{u}=B^{-} u P / P$ and $\dot{X}_{v}^{G / P}=B v P / P$. We sometimes forget the $G / P$ if there is no risk of confusion. For $u, v \in W^{P}$, we denote by $u \preccurlyeq v$ the Bruhat order if $X_{u}^{G / P} \subset X_{v}^{G / P}$. In the following lemma, we collect some well known facts about the Schubert and Richardson varieties.

Lemma 5. (i) $X_{v}^{G / P}$ is a projective variety of dimension $l(v)$ and $\operatorname{Aut}\left(X_{v}^{G / P}\right)^{\circ}$ is an algebraic group of finite dimension.

(ii) The image of $U$ in $\operatorname{Aut}\left(X_{v}^{G / P}\right)^{\circ}$ is an unipotent group denoted by $U_{v}$.

(iii) The intersection $X_{v}^{u}=X_{G / P}^{u} \cap X_{v}^{G / P}$ is an irreducible closed normal subvariety (called Richardson variety) of $X_{v}^{G / P}$ of $d i$ mension $l(v)-l(u)$, if $u \preccurlyeq v$. It is empty otherwise.

(iv) If $u \preccurlyeq v$ then $\stackrel{\circ}{X}_{G / P}^{u} \cap \dot{\circ}_{v}^{G / P}$ is a nonempty open subset contained in the smooth locus of $X_{v}^{u}$.

(v) Let $u \preccurlyeq v$ such that $l(v)=l(u)+1$. Then the Richardson variety $X_{v}^{u}$ is isomorphic to $\mathrm{P}^{1}$ and $\dot{\circ}_{G / P}^{u} \cap \stackrel{\circ}{X}_{v}^{G / P}$ is isomorphic to $\mathrm{C}^{*}$.

(vi) Assume that $u \preccurlyeq v$ and $x \in \stackrel{\circ}{X}_{G / P}^{u} \cap \dot{\circ}_{v}^{G / P}$. Then the sequence induced by the inclusions 


$$
0 \longrightarrow T_{x}\left(\stackrel{\circ}{X}_{G / P}^{u} \cap \stackrel{\circ}{X}_{v}^{G / P}\right) \longrightarrow T_{x} \stackrel{\circ}{v}_{v}^{G / P} \longrightarrow \frac{T_{x} G / P}{T_{x} \dot{X}_{G / P}^{u}} \longrightarrow 0
$$

is exact.

Proof. The normality of Richardson's varieties is proved in [Kum12, Proposition 6.5]). The last assertion is an easy consequence of [Kum02, Lemma 7.3.10]). The others assertions are banal (see [Kum02]).

Consider the homology group $\mathrm{H}_{*}(X, \mathrm{z})=\oplus_{v \in W^{P} \mathrm{Z}}\left[X_{v}^{G / P}\right]$. Then $\mathrm{H}^{*}(X, \mathrm{z}) \simeq \operatorname{Hom}\left(\mathrm{H}_{*}(X, \mathrm{z}), \mathrm{z}\right)$ has a "basis" $\left(\epsilon_{u}\right)_{u \in W^{P}}$ defined by

$$
\epsilon_{u}\left(\left[X_{v}^{G / P}\right]\right)=\delta_{v}^{u}, \quad \forall v \in W^{P} .
$$

For $u_{1}, u_{2}$, and $v \in W^{P}$, define $n_{u_{1} u_{2}}^{v} \in \mathrm{z}$ by

$$
\epsilon_{u_{1}} \cdot \epsilon_{u_{2}}=\sum_{v \in W^{P}} n_{u_{1} u_{2}}^{v} \epsilon_{v}
$$

By [KN98], $n_{u_{1} u_{2}}^{v} \geq 0$.

\subsection{Kleiman's lemma.}

Lemma 6. Let $u_{1}, u_{2}$, and $v$ in $W^{P}$ such that $u_{1} \preccurlyeq v$ and $u_{2} \preccurlyeq v$. Assume that $l(v)=l\left(u_{1}\right)+l\left(u_{2}\right)$.

For general $h \in U_{v}, X_{v}^{u_{1}} \cap h X_{v}^{u_{2}}=\stackrel{\circ}{X}_{G / P}^{u_{1}} \cap h\left(\dot{\circ}_{G / P}^{u_{2}} \cap \stackrel{\circ}{X}_{v}^{G / P}\right)$ is finite and transverse. More precisely, for any $x \in X_{v}^{u_{1}} \cap h X_{v}^{u_{2}}$ the following map induced by inclusions

$$
T_{x} X_{v}^{G / P} \longrightarrow \frac{T_{x} G / P}{T_{x} X_{G / P}^{u_{1}}} \oplus \frac{T_{x} G / P}{T_{x} \tilde{h} X_{G / P}^{u_{2}}}
$$

is an isomorphism, where $\tilde{h} \in U$ satisfies $r(\tilde{h})=h$.

Proof. It is an application of Kleiman's theorem. Indeed, consider $X_{v}=$ $\cup_{\sigma \preccurlyeq v} \dot{\circ}_{\sigma}$.

Fix $\sigma \preccurlyeq v$ such that $\sigma \neq v$. For any $h \in U_{v}$, we have $X_{v}^{u_{1}} \cap h X_{v}^{u_{2}} \cap X_{\sigma}=$ $X_{\sigma}^{u_{1}} \cap h X_{\sigma}^{u_{2}}$.

Assume first that $u_{1} \preccurlyeq \sigma$ and $u_{2} \preccurlyeq \sigma$. Since $l(\sigma)<l(v),\left(\operatorname{dim} X_{\sigma}-\right.$ $\left.\operatorname{dim} X_{\sigma}^{u_{1}}\right)+\left(\operatorname{dim} X_{\sigma}-\operatorname{dim} X_{\sigma}^{u_{2}}\right)>\operatorname{dim} X_{\sigma}$. Kleiman's theorem applied in the $U_{v}$-homogeneous space $\dot{X}_{\sigma}$ shows that $X_{v}^{u_{1}} \cap h X_{v}^{u_{2}} \cap \stackrel{\circ}{X}_{\sigma}$ is empty for general $h \in U_{v}$.

Otherwise, $X_{\sigma}^{u_{1}}$ or $X_{\sigma}^{u_{2}}$ is empty.

Similarly, for general $h \in U_{v}, \partial X^{u_{1}} \cap h X_{v}^{u_{2}}$ and $X^{u_{1}} \cap h\left(\partial X^{u_{2}} \cap X_{v}\right)$ is empty. Here $\partial X^{u}=X^{u}-\dot{o}^{u}$. Indeed, only finitely many $B^{-}$-orbits $\dot{X}^{u_{1}} \subset X^{u}$ intersect $X_{v}$.

Hence, for general $h \in U_{v}$, we have $X_{v}^{u_{1}} \cap h X_{v}^{u_{2}}=\stackrel{\circ}{X}^{u_{1}} \cap h\left(\stackrel{\circ}{X}^{u_{2}} \cap \stackrel{\circ}{X}_{v}\right)$. 
Now, by Kleiman's theorem in the $U_{v}$-homogeneous space $\stackrel{\circ}{X}_{v}$, for general $h \in U_{v}, X_{v}^{u_{1}} \cap h X_{v}^{u_{2}}=\stackrel{\circ}{X}_{G / P}^{u_{1}} \cap h\left(\stackrel{\circ}{X}_{G / P}^{u_{2}} \cap \stackrel{\circ}{X}_{v}^{G / P}\right)$ is finite and for any $x \in X_{v}^{u_{1}} \cap h X_{v}^{u_{2}}$ the map

$$
T_{x} X_{v}^{G / P} \longrightarrow \frac{T_{x} X_{v}^{G / P}}{T_{x} X_{v}^{u_{1}}} \oplus \frac{T_{x} X_{v}^{G / P}}{T_{x} h X_{v}^{u_{2}}}
$$

is an isomorphism. Since $x \in \stackrel{\circ}{X}_{v} \cap \stackrel{\circ}{X}^{u_{1}}$, Lemma 5 implies that the natural map $\frac{T_{x} X_{v}^{G / P}}{T_{x} X_{v}^{u_{1}}} \longrightarrow \frac{T_{x} G / P}{T_{x} X_{G / P}^{u_{1}}}$ is an isomorphisms. Similarly, $\frac{T_{h-1} X_{v}^{G / P}}{T_{h^{-1} X_{x}} X_{v}^{u_{2}}} \longrightarrow$ $\frac{T_{h-1} G / P}{T_{h-1} X_{G / P}^{u_{2}}}$ is an isomorphism. Since both $G / P$ and $X_{v}$ are $\tilde{h}$-stable, by applying $\tilde{h}$, one deduces that $\frac{T_{x} X_{v}^{G / P}}{T_{x} h X_{v}^{u}} \longrightarrow \frac{T_{x} G / P}{T_{x} \tilde{h} X_{G / P}^{u_{2}}}$ is an isomorphism.

Lemma 7. Let $h \in U_{v}$ satisfying Lemma 6. Then

$$
\sharp\left(X_{v}^{u_{1}} \cap h X_{v}^{u_{2}}\right)=n_{u_{1} u_{2}}^{v} .
$$

Proof. We first prove that one may assume that $B=P$. Consider the projection $\pi: G / B \longrightarrow G / P$ and the associated morphism $\pi^{*}$ : $\mathrm{H}^{*}(G / P, \mathrm{z}) \longrightarrow \mathrm{H}^{*}(G / B, \mathrm{z})$ in cohomology. Then, for any $u \in W^{P}$, $\pi^{*}\left(\epsilon_{u}(G / P)\right)=\epsilon_{u}(G / B)$. In particular, $n_{u_{1} u_{2}}^{v}(G / B)=n_{u_{1} u_{2}}^{v}(G / P)$, for any $u_{1}, u_{2}$ and $v$ in $W^{P}$.

Note that, for any $u \in W^{P}, X_{G / B}^{u}=\pi^{-1}\left(X_{G / P}^{u}\right)$ and $\pi$ maps $\stackrel{\circ}{X}_{u}^{G / B}$ bijectively onto $\dot{X}_{u}^{G / B}$. Then, for any $h$ in $U_{v}, \pi$ maps $X_{G / B}^{u_{1}} \cap h\left(X_{v}^{G / B} \cap\right.$ $\left.X_{G / B}^{u_{2}}\right)$ bijectively onto $X_{G / P}^{u_{1}} \cap h\left(X_{v}^{G / P} \cap X_{G / P}^{u_{2}}\right)$. In particular, if the lemma holds for $G / B$ it holds for $G / P$.

Let $\tilde{G}$ be the Kac-Moody group completed along the negative roots (as opposed to completed along the positive roots). Let $\tilde{B}^{-}$be the Borel subgroup of $\tilde{G}$. Let $\tilde{X}=\tilde{G} / B$ be the 'thick' flag variety which contains the standard KM-flag variety $X=G / B$. If $G$ is not of finite type, $\tilde{X}$ is an infinite dimensional non quasi-compact scheme (cf. [Ka, $\S 4])$. For $w \in W$, denote by $\tilde{X}^{w}$ the closure of $\tilde{B}^{-} w \underline{Q}$ in $\tilde{X}$. Observe that $\tilde{X}^{w} \cap X=X^{w}$. Let $K^{0}(\tilde{X})$ denote the Grothendieck group of coherent $\mathcal{O}_{\tilde{X}}$-modules (see [BK14, $\left.§ 3.5\right]$ for details). Similarly, define $K_{0}(X):=\lim _{n \rightarrow \infty} K_{0}\left(X_{n}\right)$, where $\left\{X_{n}\right\}_{n \geq 1}$ is the filtration of $X$ and $K_{0}\left(X_{n}\right)$ is the Grothendieck group of coherent sheaves on the projective variety $X_{n}$. Then, $\left\{\left[\mathcal{O}_{X_{w}}\right]\right\}_{w \in W}$ is a basis of $K_{0}(X)$ as a Z-module. Define a pairing

$\langle\rangle:, K^{0}(\tilde{X}) \otimes K_{0}(X) \rightarrow \mathrm{Z},\langle[\mathcal{S}],[\mathcal{F}]\rangle=\sum_{i}(-1)^{i} \chi\left(X_{n}, \mathcal{T}^{\mathcal{O}_{i}}{ }_{\tilde{X}}(\mathcal{S}, \mathcal{F})\right)$,

if $\mathcal{S}$ is a coherent sheaf on $\tilde{X}$ and $\mathcal{F}$ is a coherent sheaf on $X$ supported in $X_{n}$ (for some $n$ ), where $\chi$ denotes the Euler-Poincaré characteristic. 
Let $\varpi_{\alpha_{0}}, \ldots, \varpi_{\alpha_{l}}$ be characters of $T$ dual of the coroots $\alpha_{0}^{\vee}, \ldots, \alpha_{l}^{\vee}$. Set $\rho=\sum_{i=0}^{l} \varpi_{\alpha_{i}}$. Set the sheaf on $\tilde{X}$ (see [Kum12, Theorem 10.4]

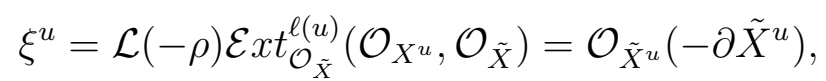

where $\partial \tilde{X}^{u}=\tilde{X}^{u}-\tilde{B}^{-} u B / B$. Then, as proved in Kum12, Proposition 3.5], for any $u, w \in W$,

$$
\left\langle\left[\xi^{u}\right],\left[\mathcal{O}_{X_{w}}\right]\right\rangle=\delta_{u, w}
$$

Let $\Delta: X \rightarrow X \times X$ be the diagonal map. Then, by [Kum12, Proposition 4.1] and [BK14, §3.5], for any $g_{1}, g_{2} \in G$

$$
n_{u_{1} u_{2}}^{w}=\sum_{i}(-1)^{i} \chi\left(\tilde{X} \times \tilde{X}, \mathcal{T}_{\text {or }}^{\mathcal{O}_{i} \tilde{X} \times \tilde{X}}\left(\xi^{u_{1}} \otimes \xi^{u_{2}},\left(g_{1}^{-1}, g_{2}^{-1}\right) \cdot \Delta_{*}\left(\mathcal{O}_{X_{v}}\right)\right)\right.
$$

Let $\tilde{h}$ in $U$ such that $r(\tilde{h})=h$.

The support of $\mathcal{T}$ or $_{i}^{\mathcal{O}_{\tilde{X} \times \tilde{X}}}\left(\xi^{u_{1}} \otimes \xi^{u_{2}},\left(1, \tilde{h}^{-1}\right) \cdot \Delta_{*}\left(\mathcal{O}_{X_{v}}\right)\right)$ is contained in $\left(\tilde{X}^{u_{1}} \times \tilde{X}^{u_{2}}\right) \cap\left(1, \tilde{h}^{-1}\right) \Delta\left(X_{v}\right)$. The assumptions on $\tilde{h}$ implies that this support is contained in $\left(\dot{\circ}^{u_{1}} \cap \dot{\circ}_{v}\right) \times\left(\dot{\circ}^{u_{2}} \cap \stackrel{\circ}{X}_{v}\right)$. In particular, this $\mathcal{T}$ or-sheaf is equal to

$$
\mathcal{T}_{\text {or }} \mathcal{O}_{\tilde{X} \times \tilde{X}}\left(\mathcal{O}_{X^{u_{1}}} \otimes \mathcal{O}_{X^{u_{2}}},\left(1, h^{-1}\right) \cdot \Delta_{*}\left(\mathcal{O}_{X_{v}}\right)\right)
$$

The support of the $\mathcal{T}$ or-sheaves in formula (7) are contained in $u_{1} \tilde{B}^{-} \underline{o} \times u_{2} \tilde{B}^{-} \underline{o}$. By [KS09, Section 8], there exists an isomorphism $\iota: u_{1} \tilde{B}^{-} \underline{\underline{Q}} \times u_{2} \tilde{B}^{-} \underline{\underline{a}} \longrightarrow \mathrm{A}^{\infty}=\mathrm{C}^{\mathrm{N}}$ such that $\tilde{B}^{-} u_{1} \underline{\underline{O}} \times \tilde{B}^{-} u_{2} \underline{\underline{O}}$ maps onto $C^{\mathrm{N}} \geq l\left(u_{1}\right)+l\left(u_{2}\right)$. Here $\mathrm{C}^{\mathrm{N}}$ denote the set $\mathrm{C}$-valued sequences view as $\operatorname{Spec}\left(\mathrm{C}\left[T_{0}, \ldots, T_{n}, \ldots\right]\right)$ and $\mathrm{C}^{\mathrm{N}} \geq l\left(u_{1}\right)+l\left(u_{2}\right)$ is the set of sequences starting with $l\left(u_{1}\right)+l\left(u_{2}\right)$ zeros. We also set $\mathrm{C}^{\mathrm{N}} \leq m:=\left\{\left(u_{k}\right) \in \mathrm{C}^{\mathrm{N}}\right.$ : $\left.u_{k}=0 \forall k>m\right\}$. Then, there exists $m \geq l\left(u_{1}\right)+l\left(u_{2}\right)$ such that $\left(u_{1} \tilde{B}^{-} \underline{o} \times u_{2} \tilde{B}^{-} \underline{o}\right) \cap\left(X_{v} \times X_{v}\right)$ is contained in $\mathrm{C}^{\mathrm{N}} \leq m$. Now, for any $i \geq 0$,

$$
\mathcal{T}_{\text {or }} \mathcal{O}_{i \tilde{X} \times \tilde{X}}\left(\mathcal{O}_{\tilde{X}_{u_{1}}} \otimes \mathcal{O}_{\tilde{X}_{u_{2}}},\left(1, h^{-1}\right) \cdot \Delta_{*}\left(\mathcal{O}_{X_{v}}\right)\right)
$$

is the pullback of

$$
\mathcal{T}_{\operatorname{or}_{i}}{ }^{\mathcal{O}} \mathbb{C}^{\mathbb{N} \leq m}\left(\mathcal{O}_{C^{\mathbb{N}} \leq m \cap C^{\mathbb{N}} \geq l\left(u_{1}\right)+l\left(u_{2}\right)},\left(\left(1, h^{-1}\right) \cdot\left(\Delta_{*}\left(\mathcal{O}_{X_{v}}\right)\right)_{\mid C^{\mathbb{N}} \leq m}\right) .\right.
$$

Since the intersection $\iota\left(\left(1, h^{-1}\right) \Delta\left(X_{v}\right) \cap\left(u_{1} \tilde{B}^{-} \underline{o} \times u_{2} \tilde{B}^{-} \underline{o}\right)\right) \cap\left(\mathrm{C}^{\mathrm{N}} \leq m \cap\right.$ $\left.\mathrm{C}^{\mathrm{N}} \geq l\left(u_{1}\right)+l\left(u_{2}\right)\right)$ is transverse in $\mathrm{C}^{\mathrm{N}} \leq m$ it follows that these $\mathcal{T}$ or-sheaves vanish for $i \geq 1$ and that $n_{u_{1} u_{2}}^{w}$ is the cardinality of this intersection. The lemma is proved. 
4.3. The case $n_{u_{1} u_{2}}^{v}=1$.

Lemma 8. We keep notation and assumptions of Lemma 6. Moreover we assume that $n_{u_{1}, u_{2}}^{v}=1$.

Then there exist a non empty open subset $\Omega$ of $U_{v}$ and a regular map

$$
\psi: \Omega \longrightarrow \stackrel{\circ}{X}_{v}^{G / P}
$$

such that

$$
\forall h \in \Omega \quad X_{v}^{u_{1}} \cap h X_{v}^{u_{2}}=\{\psi(h)\}
$$

and

$$
\psi(h) \in \stackrel{\circ}{X}_{G / P}^{u_{1}} \cap h\left(\stackrel{\circ}{X}_{G / P}^{u_{2}} \cap \stackrel{\circ}{X}_{v}^{G / P}\right) .
$$

Proof. Consider

$$
\aleph=\left\{(x, h) \in \stackrel{\circ}{X}_{v} \times U_{v}: x \in X^{u_{1}} \cap h X_{v}^{u_{2}}\right\},
$$

and the two projections $p: \aleph \longrightarrow X^{u_{1}} \cap \stackrel{\circ}{X}_{v}$ and $\pi: \aleph \longrightarrow U_{v}$.

We first prove that $\aleph$ is irreducible.

Fix $x_{0} \in X^{u_{1}} \cap \stackrel{\circ}{X}_{v}$ and set $\varsigma: U_{v} \longrightarrow \stackrel{\circ}{X}_{v}, h \longrightarrow h x$. The stabilizer of $x$ in the unipotent group $U_{v}$ is unipotent, and hence connected. Hence the fibers of $\varsigma$ are irreducible. Since $X^{u_{2}} \cap \stackrel{\circ}{X}_{v}$ is irreducible, so is $\varsigma^{-1}\left(X^{u_{2}} \cap \dot{\circ}_{v}\right)$.

Moreover for any $h \in U_{v},\left(x_{0}, h\right) \in p^{-1}\left(x_{0}\right)$ if and only if $x_{0} \in h X^{u_{2}}$ if and only if $h^{-1} \in \varsigma^{-1}\left(X^{u_{2}} \cap \dot{\circ}_{v}\right)$. Hence $p^{-1}\left(x_{0}\right)$ is nonempty and irreducible.

Since $X^{u_{1}} \cap X_{v}^{\circ}$ is also irreducible, we deduce that $\aleph$ is.

By Lemmas 6 and 7, the general fiber of $\pi$ is a singleton. Since we are working over the complex numbers, this implies that $\pi$ is birational. Then, a partial converse map $\psi$ of $\pi$ satisfies the lemma (at least, its restriction to an open subset of $h \in U_{v}$ satisfying Lemma [6).

\section{InEQUALITIES FOR $\Gamma(A)$}

In this section, we reprove [BK14, Theorem 1.1] by similar methods in the goal to introduce some useful notation. Fix once for all, a family $\varpi_{\alpha_{0}^{\vee}}, \ldots, \varpi_{\alpha_{l}^{\vee}}$ in $\mathfrak{h}_{Q}$ such that

$$
\left\langle\varpi_{\alpha_{i}^{\vee}}, \alpha_{j}\right\rangle=\delta_{i}^{j}
$$

for any $i, j \in\{0, \ldots, l\}$. Similarly fix fundamental weights $\varpi_{\alpha_{0}}, \ldots, \varpi_{\alpha_{l}}$.

Let $\tau: \mathrm{C}^{*} \longrightarrow G$ be a morphism of ind-groups. Let $\mathcal{L}$ be a $G^{-}$ linearized line bundle on $\mathrm{X}$ and $x \in \mathrm{X}$. Since $\mathrm{X}$ is ind-projective and the action of $\mathrm{C}^{*}$ on $\mathrm{X}$ is given by a morphism of ind-varieties, $\lim _{t \rightarrow 0} \tau(t) x$ exists (i.e. the morphism $\mathrm{C}^{*} \longrightarrow \mathrm{X}, t \longmapsto \tau(t) x$ extends to $\mathrm{C}$ ). Set $z=\lim _{t \rightarrow 0} \tau(t) x$. The point $z$ is fixed by $\tau\left(\mathrm{C}^{*}\right)$ and $\tau\left(\mathrm{C}^{*}\right)$ acts linearly on the fiber $\mathcal{L}_{z}$. There exists $m \in \mathrm{Z}$ such that

$$
\forall t \in \mathrm{C}^{*} \quad \forall \tilde{z} \in \mathcal{L}_{z} \quad \tau(t) \tilde{z}=t^{m} \tilde{z} .
$$


Set $\mu^{\mathcal{L}}(x, \tau)=-m$.

Proposition 1. (see [BK14, Theorem 1.1]) Let $P$ be a standard parabolic subgroup of $G$. Let $\alpha_{i}$ be a simple root that does not belong to $\Delta(P)$. Let $u_{1}, u_{2}$, and $v$ in $W^{P}$ such that $n_{u_{1}, u_{2}}^{v} \neq 0$ in $\mathrm{H}^{*}(G / P, \mathrm{z})$.

If $\left(\lambda_{1}, \lambda_{2}, \mu\right) \in \Gamma(A)$ then

$$
\left\langle\mu, v \varpi_{\alpha_{i}^{\vee}}\right\rangle \leq\left\langle\lambda_{1}, u_{1} \varpi_{\alpha_{i}^{\vee}}\right\rangle+\left\langle\lambda_{2}, u_{2} \varpi_{\alpha_{i}^{\vee}}\right\rangle .
$$

Proof. Consider $C^{+}=P u_{1}^{-1} \underline{Q}^{-} \times P u_{2}^{-1} \underline{O}^{-} \times P v^{-1} \underline{\underline{o}}$. As a locally closed subset of $\mathrm{X}$, it is an ind-variety. Set

$$
G \times{ }_{P} C^{+}:=\left\{(g P / P, x) \in G / P \times \mathrm{x}: g^{-1} x \in C^{+}\right\} .
$$

As a locally closed subset of $G / P \times \mathrm{x}$, it is an ind-variety. Consider the maps

$$
\eta: \begin{aligned}
G \times{ }_{P} C^{+} & \longrightarrow \mathrm{X} \\
(g P / P, x) & \longmapsto x
\end{aligned}
$$

and

$$
\begin{aligned}
p: G \times{ }_{P} C^{+} & \longrightarrow G / P \\
(g P / P, x) & \longmapsto g P / P .
\end{aligned}
$$

Lemma 9. Let $g_{1}, g_{2}$, and $g_{3}$ in $G$. Then

$$
p\left(\eta^{-1}\left(g_{1} \underline{\underline{O}}^{-}, g_{2} \underline{\underline{O}}^{-}, g_{3} \underline{\underline{Q}}\right)\right)=g_{1} \stackrel{\circ}{X}_{G / P}^{u_{1}} \cap g_{2} \stackrel{\circ}{X}_{G / P}^{u_{2}} \cap g_{3} \stackrel{\circ}{V}_{v}^{G / P} .
$$

Proof. The point $\left(g P / P,\left(g_{1} \underline{O}^{-}, g_{2} \underline{O}^{-}, g_{3} \underline{O}\right)\right)$ belongs to the fiber of $\eta$ if and only if

$$
\begin{array}{ll} 
& \left(g^{-1} g_{1} \underline{O}^{-}, g^{-1} g_{2} \underline{O}^{-}, g^{-1} g_{3} \underline{O}\right) \text { belongs to } \\
& P u_{1}^{-1} \underline{O}^{-} \times P u_{2}^{-1} \underline{O}^{-} \times P v^{-1} \underline{o} \\
\Longleftrightarrow & \left(g^{-1} g_{1}, g^{-1} g_{2}, g^{-1} g_{3}\right) \in P u_{1}^{-1} B^{-} \times P u_{2}^{-1} B^{-} \times P v^{-1} B \\
\Longleftrightarrow & g_{1}^{-1} g \in B^{-} u_{1} P, g_{2}^{-1} g \in B^{-} u_{2} P \text { and } g_{3}^{-1} g \in B v P \\
\Longleftrightarrow & g P / P \in g_{1} B^{-} u_{1} P / P \cap g_{2} B^{-} u_{2} P / P \cap g_{3} B v P / P .
\end{array}
$$

Consider the morphism of ind-varieties $r: U \longrightarrow U_{v} \subset \operatorname{Aut}\left(X_{v}^{G / P}\right)$ given by the action. Set $G / i^{-}=U \underline{Q}^{-}$. Since $U \longrightarrow G / B, u \longmapsto u \underline{Q}^{-}$is an open immersion, we have a morphism $p^{+}: G / B^{-} \longrightarrow U$ such that

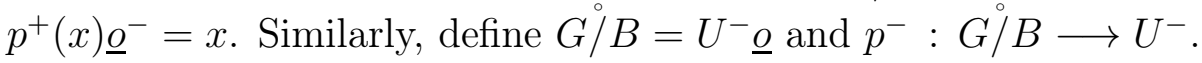

Let $\Omega \subset U_{v}$ be a nonempty open subset of $h$ 's satisfying Lemma 6 . Set

$$
\Omega_{1}=\left\{\begin{array}{ll}
\left(x_{1}, x_{2}, g_{3} \underline{\underline{o}}\right) \in \mathrm{X}: & g_{3}^{-1} x_{1} \in G / B^{-}, g_{3}^{-1} x_{2} \in G / B^{-} \text {and } \\
& r\left(p^{+}\left(g_{3}^{-1} x_{1}\right)^{-1} p^{+}\left(g_{3}^{-1} x_{2}\right)\right) \in \Omega
\end{array}\right\} .
$$

It is open and nonempty in $\mathrm{X}$. Moreover, for $\left(x_{1}, x_{2}, g_{3} \underline{o}\right) \in \Omega_{1}$, Lemma 9 implies that

$$
p\left(\eta^{-1}\left(x_{1}, x_{2}, g_{3} \underline{\underline{O}}\right)\right)=g_{3} p^{+}\left(g_{3}^{-1} x_{1}\right)\left[\stackrel{\circ}{X}^{u_{1}} \cap h\left(\stackrel{\circ}{X}^{u_{2}} \cap \stackrel{\circ}{X}_{v}\right)\right],
$$


where $h=r\left(p^{+}\left(g_{3}^{-1} x_{1}\right)^{-1} p^{+}\left(g_{3}^{-1} x_{2}\right)\right)$. By Lemmal7this fiber is nonempty.

Let $\mathcal{L}$ be the line bundle on $\mathrm{X}$ considered in Lemma 4 . Since $\left(\lambda_{1}, \lambda_{2}, \mu\right)$ belongs to $\Gamma(A)$, there exist $N>1$ and $\sigma \in \mathrm{H}^{0}\left(\mathrm{x}, \mathcal{L}^{\otimes N}\right)^{G}$ nonzero. Since $\mathrm{X}$ is irreducible, $\Omega_{1}$ intersects the nonzero locus of $\sigma$ : there exists $\mathrm{x} \in \mathrm{X}$ such that $\sigma(\mathrm{x}) \neq 0$. Since the fiber (91) is not empty, there exists $g \in G$ such that $(g P / P, \mathrm{x})$ belongs to $G \times{ }_{P} C^{+}$. Set $y=g^{-1} \mathrm{x}$. Since $\sigma$ is $G$-invariant, $\sigma(y) \neq 0$.

Let $\tau$ be a one parameter subgroup of $T$ belonging to $\oplus_{\alpha_{j} \notin \Delta(P)} \mathrm{Z}_{>0} \varpi_{\alpha_{j}^{\vee}}$. Consider

$$
\theta: \mathrm{A}^{1} \longrightarrow X, t \in \mathrm{C}^{*} \longmapsto \tau(t) y, 0 \longmapsto \lim _{t \rightarrow 0} \tau(t) y .
$$

Then $\theta^{*}(\sigma)$ is a $C^{*}$-invariant section of $\theta^{*}(\mathcal{L})$ on $\AA^{1}$. It follows that $\mu^{\mathcal{L}}(y, \tau) \leq 0$.

Let $L$ denote the standard Levi subgroup of $P$. Set $C=L u_{1}^{-1} \underline{O}^{-} \times$ $L u_{2}^{-1} \underline{\underline{O}}^{-} \times L v^{-1} \underline{\underline{o}}$. Since $y \in C^{+}, \theta(0)$ belongs to $C$. Then, a direct computation shows that

$$
\mu^{\mathcal{L}}(y, \tau)=-\left\langle\lambda_{1}, u_{1} \tau\right\rangle-\left\langle\lambda_{2}, u_{2} \tau\right\rangle+\langle\mu, v \tau\rangle \leq 0 .
$$

Since inequality (10) is fulfilled for any sufficiently large $\tau \in \oplus_{\alpha_{j} \notin \Delta(P)} \mathrm{Z}_{>0} \varpi_{\alpha_{j}^{\vee}}$, the inequality of the theorem follows by continuity.

Remark. We use notation of the proposition. Since $n_{u_{1} u_{2}}^{v}(G / P)=$ $n_{u_{1} u_{2}}^{v}(G / B)$, Proposition 1 implies that inequality 8 is fulfilled for any simple root $\alpha_{i}$, even in $\Delta(P)$.

\section{Multiplicities ON THE BOUNDARY}

In this section, we are interested in the multiplicity $c_{\lambda_{1} \lambda_{2}}^{\mu}$ for some triple $\left(\lambda_{1}, \lambda_{2}, \mu\right)$ of dominant weights such that inequality (8) is an equality. If moreover $n_{u_{1} u_{2}}^{v}=1$, we prove that $c_{\lambda_{1} \lambda_{2}}^{\mu}$ is a multiplicity for the tensor product decomposition for some strict Levi subgroup of $G$.

Theorem 5. We use notation of Proposition 1 and assume, in addition, that $n_{u_{1}, u_{2}}^{v}=1$. Let $L$ be the standard Levi subgroup of $P$. Let $\tau \in \oplus_{\alpha_{j} \notin \Delta(P)} \mathrm{Z}_{>0} \varpi_{\alpha_{j}^{\vee}}$.

Let $\left(\lambda_{1}, \lambda_{2}, \mu\right) \in\left(P_{+}\right)^{3}$ such that

$$
\left\langle\lambda_{1}, u_{1} \tau\right\rangle+\left\langle\lambda_{2}, u_{2} \tau\right\rangle=\langle\mu, v \tau\rangle .
$$

Consider the line bundle

$$
\mathcal{L}=\mathcal{L}_{-}\left(\lambda_{1}\right) \otimes \mathcal{L}_{-}\left(\lambda_{2}\right) \otimes \mathcal{L}(\mu)
$$

on $\mathrm{X}$, and

$$
C=L u_{1}^{-1} \underline{O}^{-} \times L u_{2}^{-1} \underline{O}^{-} \times L v^{-1} \underline{Q}
$$

be the closed subset of $\mathrm{x}$. 
Then, the restriction map induces an isomorphism

$$
\mathrm{H}^{0}(\mathrm{X}, \mathcal{L})^{G} \longrightarrow \mathrm{H}^{0}\left(C, \mathcal{L}_{\mid C}\right)^{L} .
$$

Before proving the theorem, we state a consequence. Set $P_{+}(L)=$ $\left\{\bar{\lambda} \in X(T):\left\langle\bar{\lambda}, \alpha_{i}^{\vee}\right\rangle \geq 0 \quad \forall \alpha_{i} \in \Delta(L)\right\}$. For any $\bar{\lambda} \in P_{+}(L)$, we have an irreducible $L$-module $L(\bar{\lambda})$ of highest weight $\bar{\lambda}$. Let $\bar{c}_{\bar{\lambda}_{1}}^{\bar{\mu}} \bar{\lambda}_{2}$ denote the multiplicities of the tensor product decomposition for the group $L$.

Corollary 1. With the notation and assumptions of the theorem, set $\bar{\lambda}_{1}=u_{1}^{-1} \lambda_{1}, \bar{\lambda}_{2}=u_{2}^{-1} \lambda_{2}$ and $\bar{\mu}=v^{-1} \mu$. We have

$$
c_{\lambda_{1} \lambda_{2}}^{\mu}=\bar{c}_{\bar{\lambda}_{1} \bar{\lambda}_{2}}^{\bar{\mu}} \text {. }
$$

Proof. Since $u_{i} \in W^{P}, u_{i}^{-1} B^{-} u_{i} \cap L=B^{-} \cap L$. Similarly, $v^{-1} B v \cap$ $L=B \cap L$. Then, the action of $L^{3}$ on $C$ allows to identify $C$ with $\mathrm{X}_{L}:=\left(L /\left(B^{-} \cap L\right)\right)^{2} \times L /(B \cap L)$. But $T$ acts with weight $u_{1}^{-1} \lambda_{1}$ on the fiber in $\mathcal{L}_{-}\left(\lambda_{1}\right)$ over $u_{1}^{-1} \underline{\underline{O}}^{-}$. We deduce that $\mathcal{L}_{\mid C}$ identifies with $\mathcal{L}_{-}\left(\bar{\lambda}_{1}\right) \otimes \mathcal{L}_{-}\left(\bar{\lambda}_{2}\right) \otimes \mathcal{L}(\bar{\mu})$ on $\mathrm{x}_{L}$. Now the corollary is obtained by applying Theorem 5 and Lemma 4 .

Note that Theorem 2 in the introduction is a particular case of Corollary 1 .

Proof of Theorem 5 up to the 5 lemmas below. We use notation of Proposition 1. Let $\bar{C}^{+}$be the closure of $C^{+}$in X. Set

$$
G \times{ }_{P} \bar{C}^{+}:=\left\{(g P / P, \mathrm{x}) \in G / P \times \mathrm{x}: g^{-1} \mathrm{x} \in \bar{C}^{+}\right\} .
$$

As a closed subset of $G / P \times \mathrm{x}$, it is an ind-variety. Consider the maps

$$
\begin{aligned}
\bar{\eta}: G \times{ }_{P} \bar{C}^{+} & \longrightarrow \mathrm{x} \\
(g P / P, \mathrm{x}) & \longmapsto \mathrm{x}
\end{aligned}
$$

and

$$
\begin{aligned}
\bar{p}: G \times{ }_{P} \bar{C}^{+} & \longrightarrow G / P \\
(g P / P, \mathrm{x}) & \longmapsto g P / P .
\end{aligned}
$$

Consider the following commutative diagram

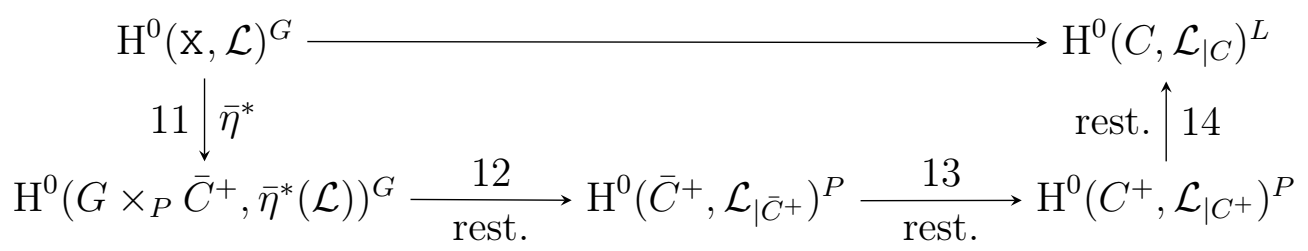

It remains to prove that the top horizontal edge is an isomorphism. But, Lemmas 11 to 14 below show that the four other morphisms are isomorphisms.

Before proving the four mentioned lemmas, we construct a partial converse map to $\bar{\eta}$. 
Lemma 10. There exists a nonempty open subset $\Omega_{1}$ of $\mathrm{X}$ such that the restriction of $\bar{\eta}$ to $\bar{\eta}^{-1}\left(\Omega_{1}\right)$ is a bijection onto $\Omega_{1}$. Moreover, the converse map $\zeta$ is a morphism of ind-varieties, mapping $\Omega_{1}$ on $G \times{ }_{P} C^{+}$.

Proof. Recall that $r: U \longrightarrow U_{v} \subset \operatorname{Aut}\left(X_{v}^{G / P}\right)^{\circ}$ denote the action. By Lemma 8 , there exist a nonempty open subset $\Omega \subset U_{v}$ and a morphism $\psi: \Omega \longrightarrow \stackrel{\circ}{X}_{v}$ such that

$$
\{\psi(h)\}=X^{u_{1}} \cap h\left(X^{u_{2}} \cap X_{v}\right) .
$$

Consider

$$
\Omega_{2}=\left\{\begin{array}{c}
\left(x_{1}, x_{2}, g_{3} \underline{\underline{O}}\right) \in \mathrm{X}: g_{3}^{-1} x_{1} \in G / \dot{\circ}^{-}, g_{3}^{-1} x_{2} \in G / B^{-}, \text {and } \\
g_{3} \underline{\underline{o}} \in G / B
\end{array}\right\} .
$$

Let $\left(x_{1}, x_{2}, x_{3}\right) \in \Omega_{2}$. Let $g_{i} \in G$ such that $g_{1} \underline{O}^{-}=x_{1}, g_{2} \underline{O}^{-}=x_{2}$ and $g_{3} \underline{\underline{O}}=x_{3}$. Observe that

$$
\begin{aligned}
& g_{1} X_{G / P}^{u_{1}} \cap g_{2} X_{G / P}^{u_{2}} \cap g_{3} X_{v}^{G / P} \\
= & g_{1} X_{G / P}^{u_{1}} \cap g_{2} X_{G / P}^{u_{2}} \cap p^{-}\left(x_{3}\right) X_{v}^{G / P} \\
= & p^{-}\left(x_{3}\right)\left(\left(p^{-}\left(x_{3}\right)^{-1} g_{1}\right) X_{G / P}^{u_{1}} \cap\left(p^{-}\left(x_{3}\right)^{-1} g_{2}\right) X_{G / P}^{u_{2}} \cap X_{v}^{G / P}\right) \\
= & p^{-}\left(x_{3}\right)\left(h_{1} X_{G / P}^{u_{1}} \cap h_{2} X_{G / P}^{u_{2}} \cap X_{v}^{G / P}\right) \\
= & \left(p^{-}\left(x_{3}\right) h_{1}\right) \cdot\left[X_{G / P}^{u_{1}} \cap h X_{G / P}^{u_{2}} \cap X_{v}^{G / P}\right],
\end{aligned}
$$

where

$$
\begin{aligned}
& h_{1}=p^{+}\left(p^{-}\left(x_{3}\right)^{-1} x_{1}\right) \\
& h_{2}=p^{+}\left(p^{-}\left(x_{3}\right)^{-1} x_{2}\right) \\
& h=h_{1}^{-1} h_{2} .
\end{aligned}
$$

Since $h \in U$,

$g_{1} X_{G / P}^{u_{1}} \cap g_{2} X_{G / P}^{u_{2}} \cap g_{3} X_{v}^{G / P}=\left(p^{-}\left(x_{3}\right) h_{1}\right) \cdot\left[X_{G / P}^{u_{1}} \cap r(h)\left(X_{G / P}^{u_{2}} \cap X_{v}^{G / P}\right)\right]$.

Let $\Omega_{1}$ be the set of $\left(x_{1}, x_{2}, x_{3}\right) \in \Omega_{2}$ such that $r(h) \in \Omega$. It is a nonempty open subset of $\mathrm{x}$. Moreover, by Lemma 8 , for $\left(x_{1}, x_{2}, x_{3}\right) \in$ $\Omega_{1}$

$$
g_{1} X_{G / P}^{u_{1}} \cap g_{2} X_{G / P}^{u_{2}} \cap g_{3} X_{v}^{G / P}=\left(p^{-}\left(x_{3}\right) h_{1}\right) \cdot\{\psi \circ r(h)\},
$$

and

$$
\left(p^{-}\left(x_{3}\right) h_{1}\right) . \psi \circ r(h) \in g_{1} \stackrel{\circ}{X}_{G / P}^{u_{1}} \cap g_{2} \stackrel{\circ}{X}_{G / P}^{u_{2}} \cap g_{3} \stackrel{\circ}{X}_{v}^{G / P} .
$$

Then the formula

$$
\left(x_{1}, x_{2}, x_{3}\right) \in \Omega_{1} \longmapsto\left(\left(p^{-}\left(x_{3}\right) h_{1}\right) . \psi \circ r(h),\left(x_{1}, x_{2}, x_{3}\right)\right)
$$

defines a morphism $\zeta$ from $\Omega_{1}$ to $G \times{ }_{P} C^{+}$such that $\eta \circ \zeta$ is the identity of $\Omega_{1}$.

Lemma 9 with $\bar{\eta}$ in place of $\eta$ and formula (13) show that the fiber of $\bar{\eta}$ over any point of $\Omega_{1}$ is a singleton. 
We now go from $\mathrm{x}$ to $G \times{ }_{P} \bar{C}^{+}$.

Lemma 11. The linear map

$$
\bar{\eta}^{*}: \mathrm{H}^{0}(\mathrm{x}, \mathcal{L}) \longrightarrow \mathrm{H}^{0}\left(G \times{ }_{P} \bar{C}^{+}, \bar{\eta}^{*}(\mathcal{L})\right)
$$

is a G-equivariant isomorphism.

Proof. Since the image of $\bar{\eta}$ contains the dense subset $\Omega_{1}$ of $\mathrm{x}, \bar{\eta}^{*}$ is injective. Fix a filtration $\mathrm{X}=\cup_{n \in \mathrm{Z} \geq 0} \mathrm{X}_{n}$ such that each $\mathrm{X}_{n}$ is a product of three finite dimensional Schubert varieties (i.e. $\left(B^{-} \times B^{-} \times B\right)$-orbit closures), $\mathrm{x}_{n}$ intersects $\Omega_{1}$ and $\mathrm{x}_{n} \subset \mathrm{x}_{n+1}$. Set $Y=G \times_{P} \bar{C}^{+}$and

$$
\stackrel{\circ}{n}_{n}:=\bar{\eta}^{-1}\left(\mathrm{X}_{n} \cap \Omega_{1}\right)=\zeta\left(\mathrm{x}_{n} \cap \Omega_{1}\right) .
$$

Let $Y_{n}$ be the closure of $\dot{Y}_{n}$ in $Y$. Then $Y_{n}$ is closed, irreducible, of finite dimension and projective.

As the image of $G \times \bar{C}^{+} \longrightarrow G \times{ }_{P} \bar{C}^{+},(g, x) \longmapsto(g P / P, g x), Y$ is ind-irreducible (see examples of Section 2.2 ). Moreover, $\cup_{n \in Z_{\geq 0}} Y_{n}$ and hence $\cup_{n \in Z} Y_{n}$ contains the nonempty open subset $\bar{\eta}^{-1}\left(\Omega_{1}\right)$. Then, Lemma 3 implies that $Y=\cup_{n \in z_{\geq 0}} Y_{n}$.

We now prove the surjectivity of $\bar{\eta}^{*}$. Let $\sigma \in \mathrm{H}^{0}\left(G \times_{P} \bar{C}^{+}, \bar{\eta}^{*}(\mathcal{L})\right)$. Consider the restriction $\bar{\eta}_{n}: Y_{n} \longrightarrow \mathrm{x}_{n}$ of $\bar{\eta}$. Then $\bar{\eta}_{n}$ is proper, birational and $\mathrm{X}_{n}$ is normal. Zariski's main theorem implies that the fibers of $\bar{\eta}_{n}$ are connected. Then

$$
\bar{\eta}_{n}^{*}: \mathrm{H}^{0}\left(\mathrm{X}_{n}, \mathcal{L}_{\mid \mathrm{x}_{n}}\right) \longrightarrow \mathrm{H}^{0}\left(Y_{n}, \bar{\eta}^{*}(\mathcal{L})_{\mid Y_{n}}\right)
$$

is an isomorphism (see e.g. [Pet04, Chap IV, Corollary 5]).

Let $\tilde{\sigma}_{n} \in \mathrm{H}^{0}\left(\mathrm{X}_{n}, \mathcal{L}_{\mid \mathrm{x}_{n}}\right)$ such that $\bar{\eta}_{n}^{*}\left(\tilde{\sigma}_{n}\right)=\sigma_{\mid Y_{n}}$. Since $\Omega_{1} \cap \mathrm{X}_{n}$ is dense in $\mathrm{X}_{n}$, the restriction of $\tilde{\sigma}_{n+1}$ to $\mathrm{X}_{n}$ is equal to $\tilde{\sigma}_{n}$. Hence $\left(\tilde{\sigma}_{n}\right)_{n \in \mathrm{N}}$ is a global section $\tilde{\sigma}$ of $\mathcal{L}$ on $\mathrm{X}$. Moreover, $\bar{\eta}^{*}(\tilde{\sigma})=\sigma$.

We now go from $G \times{ }_{P} \bar{C}^{+}$to $\bar{C}^{+}$.

Lemma 12. The linear map

$$
\mathrm{H}^{0}\left(G \times{ }_{P} \bar{C}^{+}, \bar{\eta}^{*}(\mathcal{L})\right)^{G} \longrightarrow \mathrm{H}^{0}\left(\bar{C}^{+}, \mathcal{L}_{\mid \bar{C}^{+}}\right)^{P}
$$

is an isomorphism.

Proof. Embed $\bar{C}^{+}$in $G \times{ }_{P} \bar{C}^{+}$by mapping $x \in \bar{C}^{+}$to $(P / P, x)$. Since $\bar{\eta}^{*}(\mathcal{L})_{\mid \bar{C}^{+}}=\mathcal{L}_{\mid \bar{C}^{+}}$, the map of the lemma is well defined.

Since $\bar{C}^{+}$intersects any $G$-orbit in $G \times{ }_{P} \bar{C}^{+}$, the map is injective. Let $\sigma \in \mathrm{H}^{0}\left(\bar{C}^{+}, \mathcal{L}_{\mid \bar{C}^{+}}\right)^{P}$. Set

$$
\begin{aligned}
\tilde{\sigma}: G \times{ }_{P} \bar{C}^{+} & \longrightarrow \bar{\eta}^{*}(\mathcal{L}) \\
(g P / P, x) & \longmapsto g \sigma\left(g^{-1} x\right) .
\end{aligned}
$$

Note that $\tilde{\sigma}$ is well defined as a map since $\bar{\eta}^{*}(\mathcal{L})$ is $G$-linearized and $\sigma$ is $P$-invariant. Moreover $\tilde{\sigma}$ is $G$-invariant. It is regular, since the morphism $G \longrightarrow G / P$ is locally trivial in Zariski topology, because 
of Birkhoff's decomposition. Hence $\tilde{\sigma} \in \mathrm{H}^{0}\left(G \times{ }_{P} \bar{C}^{+}, \bar{\eta}^{*}(\mathcal{L})\right)^{G}$ and $\tilde{\sigma}_{\mid \bar{C}^{+}}=\sigma$.

We now go from $\bar{C}^{+}$to $C^{+}$. Let $\tilde{\tau}: \mathrm{C}^{*} \longrightarrow T$ such that $\tilde{\tau} \in \mathrm{Z}_{>0} \tau$.

Lemma 13. Recall that $\mu^{\mathcal{L}}(C, \tau)=0$. Then the restriction map

$$
\mathrm{H}^{0}\left(\bar{C}^{+}, \mathcal{L}_{\mid \bar{C}^{+}}\right)^{\tilde{\tau}\left(\mathrm{C}^{*}\right)} \longrightarrow \mathrm{H}^{0}\left(C^{+}, \mathcal{L}_{\mid C^{+}}\right)^{\tilde{\tau}\left(\mathrm{C}^{*}\right)}
$$

is an isomorphism.

Proof. Since $C^{+}$is dense in $\bar{C}^{+}$, the restriction is injective. For $w \in W$, we recall that

$$
X_{w}^{G / B}=\overline{B w \underline{Q}} \quad X_{G / B}^{w}=\overline{B^{-} w \underline{Q}},
$$

and set

$$
X_{w}^{G / B^{-}}=\overline{B^{-} w \underline{Q}^{-}} \quad X_{G / B^{-}}^{w}=\overline{B w \underline{Q}^{-}}
$$

in such a way $\operatorname{dim}\left(X_{w}^{G / B}\right)=\operatorname{dim}\left(X_{w}^{G / B^{-}}\right)=l(w)$ and $\operatorname{codim}\left(X_{G / B}^{w}\right)=$ $\operatorname{codim}\left(X_{G / B^{-}}^{w}\right)=l(w)$. Note also that $\overline{P u_{1}^{-1} \underline{o}^{-}}=X_{G / B^{-}}^{u_{1}^{-1}}$ and $\overline{P u_{2}^{-1} \underline{o}^{-}}=$ $X_{G / B^{-}}^{u_{2}^{-1}}$, whereas $X_{v^{-1}}^{G / B}$ is finite dimensional and closed in $\overline{P v^{-1} \underline{O}}$.

Consider a sequence $\left(w_{1}^{n}, w_{2}^{n}, w_{3}^{n}\right) \in W^{3}$ such that, for $i=1,2$ and for any $n \in \mathrm{N}, u_{i}^{-1} \preccurlyeq w_{i}^{n} \preccurlyeq w_{i}^{n+1}$ and $v^{-1} \preccurlyeq w_{3}^{n} \preccurlyeq w_{3}^{n+1}$ and

$$
\bar{C}_{n}^{+}:=\left(X_{w_{1}^{n}}^{G / B^{-}} \cap X_{G / B^{-}}^{u_{1}^{-1}}\right) \times\left(X_{w_{2}^{n}}^{G / B^{-}} \cap X_{G / B^{-}}^{u_{2}^{-1}}\right) \times X_{w_{3}^{n}}^{G / B}
$$

is a filtration of $\bar{C}^{+}$. In particular $X_{w_{3}}^{G / B} \subset \overline{P v^{-1} \underline{Q}}$. Note that if $P$ has finite type, $\left(w_{3}^{n}\right)_{n \in \mathrm{N}}$ can be chosen to be constant. Note also that $C^{+} \cap \bar{C}_{n}^{+}$is open in $\bar{C}_{n}^{+}$and nonempty for any $n$. Let $\sigma \in \mathrm{H}^{0}\left(C^{+}, \mathcal{L}_{\mid C^{+}}\right)^{\tilde{\tau}}$ and let $\sigma_{n}$ denote its restriction to $C^{+} \cap \bar{C}_{n}^{+}$. We have to prove that $\sigma$ extends to a section $\bar{\sigma}$ on $\bar{C}^{+}$. It remains to prove that each $\sigma_{n}$ extends to a section $\bar{\sigma}_{n}$ on $\bar{C}_{n}^{+}$. Indeed, then $\bar{\sigma}=\left(\bar{\sigma}_{n}\right)_{n \in \mathrm{N}} \in \mathrm{H}^{0}\left(\bar{C}^{+}, \mathcal{L}_{\mid \bar{C}^{+}}\right)^{\tilde{\tau}}$ extends $\sigma$.

Fix $n \in \mathrm{N}$. By Lemma 5, $\bar{C}_{n}^{+}$is normal. Then, to prove that $\sigma_{n}$ extends to $\bar{C}_{n}^{+}$it is sufficient to prove that it has no pole along the divisors of $\bar{C}_{n}^{+}-C^{+}$. Let $D_{n}$ be such a divisor. Then, either

(a) $D_{n}=\left(X_{w_{1}^{n}} \cap X^{\tilde{u}_{1}^{-1}}\right) \times\left(X_{w_{2}^{n}} \cap X^{u_{2}^{-1}}\right) \times X_{w_{3}^{n}}$, for some $\tilde{u}_{1} \in W^{P}$ such that $u_{1}^{-1} \preccurlyeq \tilde{u}_{1}^{-1} \preccurlyeq w_{1}^{n}$ and $l\left(\tilde{u}_{1}\right)=l\left(u_{1}\right)+1$; or

(a') $D_{n}=\left(X_{w_{1}^{n}} \cap X^{u_{1}^{-1}}\right) \times\left(X_{w_{2}^{n}} \cap X^{\tilde{u}_{2}^{-1}}\right) \times X_{w_{3}^{n}}$, for some $\tilde{u}_{2} \in W^{P}$ such that $u_{2}^{-1} \preccurlyeq \tilde{u}_{2}^{-1} \preccurlyeq w_{2}^{n}$ and $l\left(\tilde{u}_{2}\right)=l\left(u_{2}\right)+1$; or

(b) $D_{n}=\left(X_{w_{1}^{n}} \cap X^{u_{1}^{-1}}\right) \times\left(X_{w_{2}^{n}} \cap X^{u_{2}^{-1}}\right) \times X_{\tilde{w}_{3}}$ for some $\tilde{w}_{3} \in W$ such that $\tilde{w}_{3} \preccurlyeq w_{3}^{n}, l\left(\tilde{w}_{3}\right)=l\left(w_{3}^{n}\right)-1$ and $X_{\tilde{w}_{3}} \subset X_{w_{3}^{n}}-P v^{-1} \underline{o}$.

In each case, we will apply Lemma 11.5 to an affine neighborhood of $D_{n}$ in $\bar{C}_{n}^{+}$. We first construct such neighborhoods and check the assumptions of Lemma 11.5. We do not consider Case (a') that is 
similar to the first one. We also skip the power $n$ from $w_{i}^{n}$. Note that the action of $\mathrm{C}^{*}$ in Lemma 11.5 is given by $\tilde{\tau}$.

Case (a). Set

$$
X=\left(X_{w_{1}} \cap X^{u_{1}^{-1}} \cap \tilde{u}_{1}^{-1} B \underline{Q}^{-}\right) \times\left(X_{w_{2}} \cap \stackrel{\circ}{X}^{u^{-1}}\right) \times \stackrel{\circ}{X}_{w_{3}},
$$

$D=D_{n} \cap X$ and $U=X-D$.

The fact that $X$ is $T$-stable is obvious. Since $\tilde{u}_{1}^{-1} B \underline{\underline{o}}^{-}, \stackrel{\circ}{X}^{-1}$ and $\stackrel{\circ}{X}_{w_{3}}$ are open in $G / B^{-}, X^{u_{2}^{-1}}$ and $X_{w_{3}}$ respectively, $X$ is open in $\bar{C}_{n}^{+}$. By [Kum02, Lemma 7.3.5], $X^{u_{1}^{-1}} \cap \tilde{u}_{1}^{-1} B \underline{O}^{-}$is affine. Then the first factor of $X$ is affine. But, Kum02, Lemma 7.3.5] also implies that the two other factors are affine, and $X$ is affine.

Moreover, by Lemma 5, $X$ is normal.

Check Assumption ( $i)$. It is sufficient to prove it for the first factor. Let $x \in\left(X_{w_{1}} \cap X^{u_{1}^{-1}} \cap \tilde{u}_{1}^{-1} B \underline{Q}^{-}\right)-X^{\tilde{u}_{1}^{-1}}$. Set $y=\lim _{t \rightarrow 0} \tilde{\tau}(t) x$. We have to prove that $y \notin\left(X_{w_{1}} \cap X^{u_{1}^{-1}} \cap \tilde{u}_{1}^{-1} B \underline{Q}^{-}\right)$.

Let $w \in W$ such that $x \in \stackrel{\circ}{X}_{G / B^{-}}^{w}$. Since $x \in X^{u_{1}^{-1}}, u_{1}^{-1} \leq w$. Since $x \in \tilde{u}_{1}^{-1} B \underline{o}^{-}, \tilde{u}_{1}^{-1} \underline{\underline{o}}^{-}$belongs to $X_{G / B^{-}}^{w}$ and $w \preccurlyeq \tilde{u}_{1}^{-1}$. But $l\left(\tilde{u}_{1}\right)=$ $l\left(u_{1}\right)+1$, hence $w=\tilde{u}_{1}^{-1}$ or $u_{1}^{-1}$. Now, $x \notin X^{\tilde{u}_{1}^{-1}}$ and $w=u_{1}^{-1}$.

Now, Lemma 11.1 implies that $y$ does not belong to $\tilde{u}_{1}^{-1} B \underline{o}^{-}$. The claim is proved.

Check Assumption (ii). We work successively on each factor of $X$.

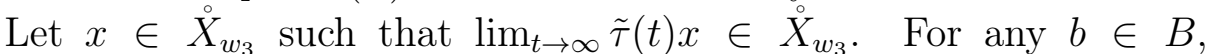
$\lim _{t \rightarrow \infty} \tilde{\tau}(t) b \tilde{\tau}\left(t^{-1}\right)$ exists in $B$. Then $\lim _{t \rightarrow 0} \tilde{\tau}(t) x \in \stackrel{\circ}{X}_{w_{3}}$. If $x$ is not fixed by $\tau\left(\mathrm{C}^{*}\right), \overline{\tilde{\tau}\left(\mathrm{C}^{*}\right) x}$ is isomorphic to $\mathrm{P}^{1}$. Hence it cannot be contained in the affine variety $\dot{\circ}_{w_{3}}$. Hence $y \notin \stackrel{\circ}{X}_{w_{3}}$. Contradiction. It follows that $x$ is fixed by $\tilde{\tau}\left(\mathrm{C}^{*}\right)$.

Similarly for $x \in X_{w_{2}} \cap \stackrel{\circ}{X}^{u_{2}^{-1}}$, if $\lim _{t \rightarrow \infty} \tilde{\tau}(t) x \in \dot{X}^{u_{2}^{-1}}$ then $x$ is fixed by $\tilde{\tau}\left(\mathrm{C}^{*}\right)$.

Now, it is sufficient to prove Assumption (ii) for the first factor of $X$. Let $x_{1}, x_{2} \in\left(X_{w_{1}} \cap X^{u_{1}^{-1}} \cap \tilde{u}_{1}^{-1} B \underline{O}^{-}\right)-X^{\tilde{u}_{1}^{-1}}$ such that $y:=$ $\lim _{t \rightarrow \infty} \tilde{\tau}(t) x_{1}=\lim _{t \rightarrow \infty} \tilde{\tau}(t) x_{2} \in D$.

We already noticed that $x_{1}$ and $x_{2}$ belong to $\dot{X}^{u_{1}^{-1}}$. By assumption, $y \in \tilde{u}_{1}^{-1} B \underline{Q}^{-} \cap X^{\tilde{u}_{1}^{-1}}=\stackrel{\circ}{X}^{\tilde{u}_{1}^{-1}}$. Then Lemma 11.2 shows that $\tilde{\tau}\left(\mathrm{C}^{*}\right) x_{1}=$ $\tilde{\tau}\left(\mathrm{C}^{*}\right) x_{2}$.

Check Assumption (iii). This can be proved component by component.

Let $x \in \stackrel{\circ}{X}_{w_{3}}$ (resp. $X_{w_{2}} \cap \stackrel{\circ}{X}^{u_{2}^{-1}}$ ). We already observe that $\lim _{t \rightarrow 0} \tilde{\tau}(t) x$ belongs to $\stackrel{\circ}{X}_{w_{3}}$ (resp. $\left.X_{w_{2}} \cap \stackrel{\circ}{X}^{u_{2}^{-1}}\right)$.

Let now $x \in\left(X_{w_{1}} \cap X^{\tilde{u}_{1}^{-1}} \cap \tilde{u}_{1}^{-1} B \underline{o}^{-}\right)=\left(X_{w_{1}} \cap \dot{X}^{\tilde{u}_{1}^{-1}}\right)$. Hence, we are in the situation of the second factor. 
Finally, for any $x \in D, \lim _{t \rightarrow 0} \tilde{\tau}(t) x$ belongs to $D$.

Check Assumption (iv). It is sufficient to consider the first factor. Let $y \in\left(X_{w_{1}} \cap X^{\tilde{u}_{1}^{-1}} \cap \tilde{u}_{1}^{-1} B \underline{Q}^{-}\right)^{\tilde{\tau}}=\left(X_{w_{1}} \cap \dot{X}^{\tilde{u}_{1}^{-1}}\right)^{\tilde{\tau}}$. We have to find $x \in\left(X_{w_{1}} \cap X^{u_{1}^{-1}} \cap \tilde{u}_{1}^{-1} B \underline{Q}^{-}\right)-X^{\tilde{u}_{1}^{-1}}$ such that $\lim _{t \rightarrow \infty} \tilde{\tau}(t) x=y$.

Assume first that $y=y_{0}=\tilde{u}_{1}^{-1} \underline{o}^{-}$. Since $l\left(\tilde{u}_{1}\right)=l\left(u_{1}\right)+1, X_{\tilde{u}_{1}^{-1}} \cap$ $X^{u_{1}^{-1}}$ is a Richardson variety of dimension one. By Lemma 5, $\dot{X}_{\tilde{u}_{1}^{-1}} \cap$ $\stackrel{\circ}{X}^{u_{1}^{-1}}$ is dense in $X_{\tilde{u}_{1}^{-1}}^{u_{-1}^{-1}}$. Let $x_{0} \in \stackrel{\circ}{X}_{\tilde{u}_{1}^{-1}} \cap \stackrel{\circ}{X}^{u_{1}^{-1}}$.

Since $\tilde{u}_{1} \in W^{P}, \tilde{u}_{1}^{-1} B^{-} \tilde{u}_{1} \cap L=B^{-} \cap L$. Moreover, $B^{-}=P^{u,-}\left(B^{-} \cap\right.$ $L)$. Hence

$$
\stackrel{\circ}{X}_{\tilde{u}_{1}^{-1}}=B^{-} \tilde{u}_{1}^{-1} \underline{Q}^{-}=P^{u,-}\left(B^{-} \cap L\right) \tilde{u}_{1}^{-1} \underline{Q}^{-}=P^{u,-} \tilde{u}_{1}^{-1} \underline{Q}^{-} .
$$

In particular, $\lim _{t \rightarrow \infty} \tilde{\tau}(t) x_{0}=y_{0}$.

Since $\tilde{u}_{1}^{-1} \preccurlyeq w_{1}, x_{0} \in X_{w_{1}}$. Moreover, $x_{0} \in \stackrel{\circ}{X}^{u_{1}^{-1}}$; thus $x_{0} \notin X^{\tilde{u}_{1}^{-1}}$. And $x_{0} \in{\stackrel{\circ}{\tilde{u}_{1}^{-1}}} \subset \tilde{u}^{-1} B \underline{o}^{-}$. Finally, $x_{0} \in\left(X_{w_{1}} \cap X^{u_{1}^{-1}} \cap \tilde{u}_{1}^{-1} B \underline{o}^{-}\right)-X^{\tilde{u}_{1}^{-1}}$.

Now $y \in\left(\stackrel{\circ}{X}^{\tilde{u}_{1}^{-1}}\right)^{\tilde{\tau}}=(B \cap L) \tilde{u}_{1}^{-1} \underline{o}^{-}$. Let $l \in B \cap L$ such that $y=l y_{0}$. Set $x=l x_{0}$. Since $l \in L, \lim _{t \rightarrow \infty} \tilde{\tau}(t) x=y$. Since $l \in L$ and $X^{u_{1}^{-1}}$ and $X^{u_{1}^{-1}}$ are $L$-stable, $x \in X^{u_{1}^{-1}}-X^{\tilde{u}_{1}^{-1}}$. Since $\tilde{u}_{1} \in W^{P}, \tilde{u}_{1}^{-1} B \tilde{u}_{1} \cap L=$ $B \cap L$. Hence $x \in \tilde{u}_{1}^{-1} B \underline{O}^{-}$. Recall that $x_{0} \in P^{u,-} y_{0}$. Since $l$ normalizes $P^{u,-}$, this emplies that $x \in P^{u,-} y$. But $X_{w_{1}}$ is $B^{-}$-stable, and $x \in X_{w_{1}}$. Finally, $x$ works.

Case (b). Set

$$
X=\left(X_{w_{1}} \cap \dot{\circ}^{u_{1}^{-1}}\right) \times\left(X_{w_{2}} \cap \stackrel{\circ}{X}^{-1}\right) \times\left(X_{w_{3}} \cap \tilde{w}_{3} B^{-} \underline{o}\right),
$$

$D=D_{n} \cap X$ and $U=X-D$.

Lemma 5 and [Kum02, Lemma 7.3.5] imply that $X$ is open in $\bar{C}^{+}$, $T$-stable, affine and normal.

Check Assumption $(i)$. It is sufficient to prove it for the third factor. Let $x \in\left(X_{w_{3}} \cap \tilde{w}_{3} B^{-} \underline{o}\right)-X^{\tilde{w}_{3}}$. Set $y=\lim _{t \rightarrow 0} \tilde{\tau}(t) x$. We have to prove that $y \notin \tilde{w}_{3} B^{-} \underline{o}$.

Like in Case a - $(i)$, one has $x \in \stackrel{\circ}{X}_{w_{3}}$. Hence $y \in(B \cap L)$. $w_{3} \underline{\underline{o}}$. But $\left(\tilde{w}_{3} B^{-} \underline{o}\right)^{\tilde{\tau}}=\left(\left(\tilde{w}_{3} B^{-} \tilde{w}_{3}^{-1}\right) \cap L\right) \tilde{w}_{3} \underline{\underline{o}}$. In particular, if $y \in \tilde{w}_{3} B^{-} \underline{\underline{o}}$ then $P v^{-1} \underline{Q}=P w_{3} \underline{Q}=P \tilde{w}_{3} \underline{\underline{O}}$. Contradiction.

Check Assumption (ii). Let $x_{1}, x_{2} \in U$ such that $\lim _{t \rightarrow \infty} \tilde{\tau}(t) x_{1}=$ $\lim _{t \rightarrow \infty} \tilde{\tau}(t) x_{2} \in D$. In the proof of Case a - $(i i)$, we proved that the two first components of each $x_{i}$ is fixed by $C^{*}$. We assume now that $x_{1}, x_{2} \in\left(X_{w_{3}} \cap \tilde{w}_{3} B^{-} \underline{o}\right)-X^{\tilde{w}_{3}}$.

Since $X_{w_{3}} \cap \tilde{w}_{3} B^{-} \underline{Q}$ is contained in $\stackrel{\circ}{X}_{w_{3}} \cup \stackrel{\circ}{X}_{\tilde{w}_{3}}, x_{1}, x_{2} \in \stackrel{\circ}{X}_{w_{3}}$. Similarly $y \in \stackrel{\circ}{X}_{\tilde{w}_{3}}$. Then Lemma 11.2 implies that $\tilde{\tau}\left(\mathrm{C}^{*}\right) x_{1}=\tilde{\tau}\left(\mathrm{C}^{*}\right) x_{2}$. 
Check Assumption (iii). We can work on each component separately. The two first one was treated in Case a- $($ iii $)$. The last one works since $X_{\tilde{w}_{3}} \cap \tilde{w}_{3} B^{-} \underline{o}=\stackrel{\circ}{X}_{\tilde{w}_{3}}$.

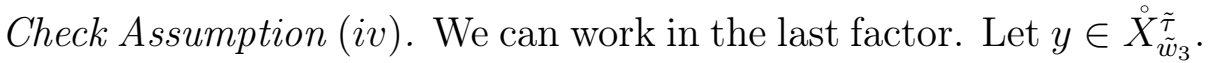

Assume first that $y=y_{0}=\tilde{w}_{3} \underline{\underline{O}}$. Consider the Richardson variety $C=X_{w_{3}} \cap X^{\tilde{w}_{3}}$ of dimension one. Pick $x_{0} \in \stackrel{\circ}{X}_{w_{3}} \cap \stackrel{\circ}{X}^{\tilde{w}_{3}}$. Then $x_{0} \in X_{w_{3}} \cap \tilde{w}_{3} B^{-} \underline{o}$. Let $\gamma$ be the character of the action of $T$ on $T_{y_{0}} C$. It is a root of $\mathfrak{g}$. Since $\overline{P y_{0}}$ does not contain $w_{3} \underline{\underline{Q}}, \gamma$ is not a root of $P$. Then $\gamma$ is a root of $P^{u,-}$. This implies that $\lim _{t \rightarrow \infty} \tilde{\tau}(t) x_{0}=y_{0}$.

Now $y \in \stackrel{\circ}{X}_{\tilde{w}_{3}}$ and there exists $l \in B \cap L$ such that $y=l y_{0}$. Consider the curve $l C$ : it is $\tilde{\tau}\left(C^{*}\right)$-stable, contained in $X_{w_{3}}$ and contains $y$. Since $X$ is open in $X_{w_{3}}, C \cap X_{w_{3}} \cap \tilde{w}_{3} B^{-} \underline{o}$ is a nonempty open subset of $C$. Then $l x \in X$. But $\lim _{t \rightarrow \infty} \tilde{\tau}(t) x=y$.

The line Bundle on the Affine subvarieties. Since $\mathcal{L}_{-}\left(\lambda_{1}\right)$ is $G$-linearized and the action of $U$ on $\underline{o}^{-}$is free, $\mathcal{L}_{-}\left(\lambda_{1}\right)$ is trivial as a line bundle on $U_{\underline{\underline{O}}}{ }^{-}$. Similarly $\mathcal{L}(\mu)$ is trivial on $U^{-} \underline{\underline{o}}$. As a consequence, $\mathcal{L}$ is trivial as a line bundle, on each affine variety $X$ we have considered.

To determine $\mathcal{L}_{\mid X}$ as a $\mathrm{C}^{*}$-linearized line bundle, it is sufficient to determine the action of $\mathrm{C}^{*}$ on one $\mathrm{C}^{*}$-fixed point. Set

$$
x_{0}=\left(u_{1}^{-1} \underline{\underline{o}}^{-}, u_{2}^{-1} \underline{\underline{o}}^{-}, w_{3} \underline{\underline{o}}\right),
$$

and

$$
\begin{aligned}
& x_{a}=\left(\tilde{u}_{1}^{-1} \underline{\underline{O}}^{-}, u_{2}^{-1} \underline{\underline{O}}^{-}, w_{3} \underline{\underline{O}}\right), \quad \text { and } \\
& x_{b}=\left(u_{1}^{-1} \underline{\underline{O}}^{-}, u_{2}^{-1} \underline{\underline{O}}^{-}, \tilde{w}_{3} \underline{\underline{O}}\right) .
\end{aligned}
$$

By assumption, $\mathrm{C}^{*}$ acts trivially on the fiber $\mathcal{L}_{x_{0}}$. In Case (a), we have constructed a copy of $\mathrm{P}^{1}$, containing $x_{0}$ and $x_{a}$ such that for $x \in \mathrm{P}^{1}-\left\{x_{0}\right\}, \lim _{t \rightarrow \infty} \tilde{\tau}(t) x=x_{a}$. Moreover, $\mathcal{L}_{\mathrm{P} 1}$ is nonnegative as a line bundle. Now, a computation in $\mathrm{P}^{1}$ shows that the action of $\mathrm{C}^{*}$ on $\mathcal{L}_{x_{a}}$ is given by a nonpositive weight $k_{a}$. $k_{b}$.

Similarly, the action of $\mathrm{C}^{*}$ on $\mathcal{L}_{x_{b}}$ is given by a nonpositive weight

Since $\mathcal{L}$ is trivial on $X$, we deduce that, for any consider affine open subsets $X$, we have

$$
H^{0}(X, \mathcal{L})^{\tilde{\tau}} \simeq \mathrm{C}[X]^{(k)},
$$

for some nonnegative integer $k$.

We are now in position to apply Lemma 11.5. By assumption the restriction $\left(\sigma_{n}\right)_{\mid U}$ belongs to $H^{0}(U, \mathcal{L})^{\tilde{\tau}} \simeq \mathrm{C}[U]^{(k)}$. By Lemma 11.5, $\mathrm{C}[U]^{(k)}=\mathrm{C}[X]^{(k)}$. Hence $\left(\sigma_{n}\right)_{\mid U}$ extends to a regular section on $X$. In particular, it has no pole along $D_{n}$. Then $\sigma_{n}$ extends to a regular section on $\bar{C}_{n}^{+}$by normality. This ends the proof of the lemma.

The last step goes from $C^{+}$to $C$. 
Lemma 14. Recall that $\mu^{\mathcal{L}}(C, \tilde{\tau})=0$. Then the restriction map

$$
\mathrm{H}^{0}\left(C^{+}, \mathcal{L}_{\mid C^{+}}\right)^{\tilde{\tau}} \longrightarrow \mathrm{H}^{0}\left(C, \mathcal{L}_{C}\right)^{\tilde{\tau}}
$$

is an isomorphism.

Proof. We first prove the injectivity. Let $\sigma \in \mathrm{H}^{0}\left(C^{+}, \mathcal{L}_{\mid C^{+}}\right)^{\tilde{\tau}}$ such that $\sigma_{\mid C}=0$ and $x \in C^{+}$. Consider the morphism

$$
\begin{aligned}
& \theta_{x}: \mathrm{C} \longrightarrow C^{+} \\
& t \longmapsto \tilde{\tau}(t) x \quad \text { if } t \neq 0 \text {, } \\
& 0 \longmapsto \lim _{t \rightarrow 0} \tilde{\tau}(t) x \text {. }
\end{aligned}
$$

It is $\mathrm{C}^{*}$-equivariant for the natural actions of $\mathrm{C}^{*}$. Moreover, $\theta_{x}^{*}(\mathcal{L})$ is trivial as $\mathrm{C}^{*}$-linearized line bundle, since $\mu^{\mathcal{L}}(C, \tilde{\tau})=0$. But $\theta_{x}^{*}(\sigma)(0)=$ 0 and $\theta_{x}^{*}(\sigma)$ is $\mathrm{C}^{*}$-invariant. Thus $\theta_{x}^{*}(\sigma)=0$. In particular $\sigma(x)=0$.

Consider now the map $\pi: C^{+} \longrightarrow C, x \longmapsto \lim _{t \rightarrow 0} \tilde{\tau}(t) x$. We claim that $\pi^{*}\left(\mathcal{L}_{\mid C}\right)$ is isomorphic to $\mathcal{L}$.

We can work on each factor of $C^{+}$separately. So assume for proving the claim that $X=G / B, C^{+}=P v^{-1} \underline{o}$ and $C=L v^{-1} \underline{o}$. Let $\iota_{\mu}$ : $X \longrightarrow \mathrm{P}(L(\mu))$ induced by the action of $G$ on the highest weight line of $\mathrm{P}(L(\mu))$. Set $k=\left\langle\tilde{\tau}, v^{-1} \mu\right\rangle$. Recall that $L(\mu)$ has a weight space decomposition $L(\mu)=\oplus_{\chi \in X(T)} L(\mu)_{\chi}$. Set

$$
L(\mu)^{k}=\bigoplus_{\langle\chi, \tilde{\tau}\rangle=k} L(\mu)_{\chi} \quad L(\mu)^{>k}=\bigoplus_{\langle\chi, \tilde{\tau}\rangle>k} L(\mu)_{\chi}
$$

Define

$$
\mathcal{E}=\left\{\left[v_{0}+v_{+}\right]: v_{0} \in L(\mu)^{k}-\{0\} \text { and } v_{+} \in L(\mu)^{>k}\right\}
$$

as a subset of $\mathrm{P}\left(L(\mu)^{k} \oplus L(\mu)^{>k}\right)$ and so of $\mathrm{P}(L(\mu))$. Then $\iota_{\mu}(C)$ is contained in $\mathrm{P}\left(L(\mu)^{k}\right)$ and $\iota_{\mu}\left(C^{+}\right)$is contained in $\mathcal{E}$. Moreover, $\pi$ is the restriction of the canonical projection $\mathcal{E} \longrightarrow \mathrm{P}\left(L(\mu)^{k}\right)$. But, $\mathcal{L}(\mu)^{*}$ is the restriction to $X$ of the tautological bundle on $\mathrm{P}(L(\mu))$. The claim follows.

Let us prove the surjectivity. Let $\sigma \in \mathrm{H}^{0}\left(C, \mathcal{L}_{\mid C}\right)^{\tilde{\tau}}$. By the claim, we have to prove that $\sigma$ extends to a $\mathrm{C}^{*}$-invariant section of $\pi^{*}(\mathcal{L})$. The morphism

$$
\begin{aligned}
C^{+} & \longrightarrow \mathcal{L}_{\mid C} \\
x & \longmapsto \sigma(\pi(x))
\end{aligned}
$$

induces such an extension.

\section{The Belkale-Kumar-Brown Product}

In this section, we purpose a construction of the $\mathrm{BKB}$ product $\odot_{0}$ (see [BK14]) and prove some properties of it. 
7.1. Preliminaries of linear algebra. Let $E$ be a complex vector space filtered by linear subspaces

$$
\{0\}=E^{0} \subset E^{1} \subset E^{2} \subset \cdots \subset E^{n} \subset \cdots
$$

such that $E=\cup_{n} E^{n}$. Let $T$ and $U$ be two linear subspaces of $E$ such that $T$ has finite dimension, $U$ has finite codimension and $\operatorname{dim}(T)=$ $\operatorname{codim}(U)$. Consider the linear map

$$
\begin{aligned}
\Theta: E & \longrightarrow E / T \oplus E / U \\
v & \longmapsto(v+T, v+U) .
\end{aligned}
$$

Define the induced filtrations from $E$ to $T$ and $E / U$ :

$$
T^{n}=E^{n} \cap T \quad \text { and } \quad(E / U)^{n}=E^{n} /\left(U \cap E^{n}\right) .
$$

Set

$$
\delta=\sum_{n>0} n\left(\operatorname{dim}\left(T^{n} / T^{n-1}\right)-\operatorname{dim}\left((E / U)^{n} /(E / U)^{n-1}\right)\right) .
$$

Lemma 15. If $\Theta$ is an isomorphism then

$$
\operatorname{dim}\left(T^{n}\right) \leq \operatorname{dim}(E / U)^{n} \quad \forall n \in \mathrm{Z}_{\geq 0} .
$$

In particular $\delta \geq 0$.

Proof. Consider

$$
\begin{aligned}
\bar{\Theta}: T & \longrightarrow E / U \\
v & \longmapsto v+U .
\end{aligned}
$$

Since $\Theta$ is an isomorphism, so is $\bar{\Theta}$. Moreover $\bar{\Theta}\left(T^{n}\right) \subset(E / U)^{n}$. Then the first inequality of the lemma is a consequence of the injectivity of the restriction of $\bar{\Theta}$ to $T^{n}$.

Since $T$ and $E / U$ are finite dimensional, there exists $N$ such that $T^{n}=T$ and $(E / U)^{n}=E / U$ for any $n \geq N$. Then

$$
\begin{aligned}
\delta & =\sum_{n=1}^{N} n\left(\operatorname{dim}\left(T^{n} / T^{n-1}\right)-\operatorname{dim}\left((E / U)^{n} /(E / U)^{n-1}\right)\right) \\
& \left.=\sum_{n=1}^{N} n\left(\operatorname{dim}\left(T^{n}\right)-\operatorname{dim}\left(T^{n-1}\right)-\operatorname{dim}\left((E / U)^{n}\right)+\operatorname{dim}(E / U)^{n-1}\right)\right) \\
& =\sum_{n=0}^{N-1} \operatorname{dim}\left((E / U)^{n}\right)-\operatorname{dim}\left(T^{n}\right),
\end{aligned}
$$

since $\operatorname{dim}(T)=\operatorname{dim}(E / U)$. In particular, $\delta \geq 0$.

Consider the graded vector spaces $\operatorname{gr} T=\oplus_{n \in \mathrm{Z}_{>0}} T^{n} / T^{n-1}$ and $\operatorname{gr}(E / U)=\oplus_{n \in \mathrm{Z}_{>0}}(E / U)^{n} /(E / U)^{n-1}$.

The map $\bar{\Theta}$ induces a graded linear map

$$
\operatorname{gr} \bar{\Theta}: \operatorname{gr} T \longrightarrow \operatorname{gr}(E / U) \text {. }
$$

Lemma 16. Assume that $\Theta$ is an isomorphism. The following assertions are equivalent

(i) $\operatorname{gr} \bar{\Theta}$ is an isomorphism;

(ii) $\operatorname{dim}\left(T^{n}\right)=\operatorname{dim}(E / U)^{n} \quad \forall n \in \mathrm{Z}_{\geq 0}$; 
(iii) $\delta=0$.

Proof. The second assertion implies the last one by the proof of Lemma15. If $\operatorname{gr} \bar{\Theta}$ is an isomorphism then for any $n, \operatorname{dim}\left(T^{n}\right)-\operatorname{dim}\left(T^{n-1}\right)=$ $\operatorname{dim}(E / U)^{n}-\operatorname{dim}(E / U)^{n-1}$. Since $T^{0}$ and $(E / U)^{0}$ are trivial the equalities of assertion (iii) follow.

Assume now that $\delta=0$. Since $\delta=\sum_{n>0} \operatorname{dim}(E / U)^{n}-\operatorname{dim}\left(T^{n}\right)$, Lemma 15] shows that $\operatorname{dim}(E / U)^{n}=\operatorname{dim}\left(T^{n}\right)$, for any $n$. Then, the injectivity of $\Theta$ implies that $\bar{\Theta}$ induces isomorphisms from $T^{n}$ onto $(E / U)^{n}$, for any $n$. It follows that $\operatorname{gr} \bar{\Theta}$ is an isomorphism.

7.2. Definition of the BKB product. Let $P$ be a standard parabolic subgroup of $G$. Let $u_{1}, u_{2}$, and $v$ in $W^{P}$ such that $l(v)=l\left(u_{1}\right)+l\left(u_{2}\right)$ and $n_{u_{1} u_{2}}^{v} \neq 0$. Set

$$
\begin{gathered}
\mathcal{T}=T_{P / P} G / P \\
\mathcal{T}^{u_{1}}=T_{P / P} u_{1}{ }^{-1} X_{G / P}^{u_{1}} \\
\mathcal{T}^{u_{2}}=T_{P / P} u_{2}^{-1} X_{G / P}^{u_{2}}
\end{gathered}
$$

Fix $\tau$ be a one parameter subgroup of $T$ belonging to $\oplus_{\alpha_{j} \notin \Delta(P)} \mathrm{Z}_{>0} \varpi_{\alpha_{j}^{\vee}}$. Observe that $P$ acts on $\mathcal{T}$. Under the action of $\tau, \mathcal{T}$ decomposes as $\mathcal{T}=\bigoplus_{n \in \mathrm{Z}} \mathcal{T}_{n}$, where $\mathcal{T}_{k}=\left\{\xi \in \mathcal{T}: \tau(t) \xi=t^{k} \xi \quad \forall t \in \mathrm{C}^{*}\right\}$. Note that $\mathcal{T}_{n}=\{0\}$ for all $n \geq 0$. Set

$$
\mathcal{T}^{n}=\oplus_{k \leq n} \mathcal{T}_{-k} .
$$

Then $\left(\mathcal{T}^{n}\right)_{n \in \mathrm{Z} \geq 0}$ forms a $P$-stable filtration of $\mathcal{T}$. Moreover $\mathcal{T}^{0}=\{0\}$. Consider also the induced filtrations $\left(\mathcal{T} / \mathcal{T}^{u_{1}}\right)^{n},\left(\mathcal{T} / \mathcal{T}^{u_{2}}\right)^{n}$ and $\mathcal{T}_{v}^{n}$ on $\mathcal{T} / \mathcal{T}^{u_{1}}, \mathcal{T} / \mathcal{T}^{u_{2}}$, and $\mathcal{T}_{v}$. Set

$$
\begin{aligned}
\delta_{u_{1} u_{2}}^{v}=\sum_{n \geq 0} n\left(\operatorname{dim}\left(\mathcal{T}_{v}^{n} / \mathcal{T}_{v}^{n-1}\right)\right. & -\operatorname{dim}\left(\left(\left(\mathcal{T} / \mathcal{T}^{u_{1}}\right)^{n}\right) /\left(\mathcal{T} / \mathcal{T}^{u_{1}}\right)^{n-1}\right) \\
& \left.-\operatorname{dim}\left(\left(\left(\mathcal{T} / \mathcal{T}^{u_{2}}\right)^{n}\right) /\left(\mathcal{T} / \mathcal{T}^{u_{2}}\right)^{n-1}\right)\right) .
\end{aligned}
$$

Lemma 17. If $n_{u_{1} u_{2}}^{v} \neq 0$ then $\delta_{u_{1} u_{2}}^{v} \geq 0$.

Proof. Consider the map

$$
\eta: G \times{ }_{P} C^{+} \longrightarrow \mathrm{x},
$$

as in the proof of Proposition 1.

By Lemma 6, there exists $b \in B$ such that $X_{v}^{u_{1}} \cap b X_{v}^{u_{2}}=\stackrel{\circ}{X}_{v}^{G / P} \cap$ $\stackrel{\circ}{X}_{G / P}^{u_{1}} \cap b \dot{X}_{G / P}^{u_{2}}$ is transverse and nonempty by Lemma 7 . Let $g \in G$ such that $g P / P$ belongs to this intersection. There exist $p_{1}, p_{2}$ and $p_{3}$ in $P$ such that

$$
\left(g P / P,\left(g p_{1} u_{1}^{-1} \underline{Q}^{-}, g p_{2} u_{2}^{-1} \underline{O}^{-}, g p_{3} v^{-1} \underline{o}\right)\right)
$$

belongs to the fiber $\eta^{-1}\left(\underline{o}^{-}, b \underline{o}^{-}, \underline{o}\right)$. Observe that

$$
g\left(p_{1} u_{1}^{-1} X_{G / P}^{u_{1}} \cap p_{2} u_{2}^{-1} X_{G / P}^{u_{2}} \cap p_{3} v^{-1} X_{v}^{G / P}\right)=X_{v}^{u_{1}} \cap b X_{v}^{u_{2}}
$$


is transverse. By Lemma 6, the canonical map

$$
p_{3} \mathcal{T}_{v} \longrightarrow \frac{\mathcal{T}}{p_{1} \mathcal{T}^{u_{1}}} \oplus \frac{\mathcal{T}}{p_{2} \mathcal{T}^{u_{2}}}
$$

is an isomorphism.

The lemma follows by applying Lemma 15 with $E=\mathcal{T} \oplus \mathcal{T}, E^{n}=$ $\mathcal{T}^{n} \oplus \mathcal{T}^{n}, U=p_{1} \mathcal{T}^{u_{1}} \oplus p_{2} \mathcal{T}^{u_{2}}$ and $T \simeq \mathcal{T}_{v}$ embedded in $E$ by $x \mapsto$ $\left(p_{3} x, p_{3} x\right)$.

For $w \in W$, we denote by $\Phi_{w}=w^{-1} \Phi^{+} \cap \Phi^{-}$the inversion set of $w$. Then, $\Phi_{w}$ consists in $l(w)$ real roots. Recall that $\rho=\sum_{i=0}^{l} \varpi_{\alpha_{i}}$.

Lemma 18. With above notation, we have

$$
\delta_{u_{1} u_{2}}^{v}=\left\langle-v^{-1} \rho+u_{1}^{-1} \rho+u_{2}^{-1} \rho-\rho, \tau\right\rangle .
$$

Proof. Since

$$
\mathcal{T} / \mathcal{T}^{u_{1}} \simeq \oplus_{\alpha \in \Phi_{u_{1}}} \mathfrak{g}_{\alpha} \quad \mathcal{T} / \mathcal{T}^{u_{2}} \simeq \oplus_{\alpha \in \Phi_{u_{2}}} \mathfrak{g}_{\alpha} \quad \text { and } \quad \mathcal{T}_{v} \simeq \oplus_{\alpha \in \Phi_{v}} \mathfrak{g}_{\alpha}
$$

we have

$$
\delta_{u_{1} u_{2}}^{v}=-\sum_{\alpha \in \Phi_{v}}\langle\alpha, \tau\rangle+\sum_{\alpha \in \Phi_{u_{1}}}\langle\alpha, \tau\rangle+\sum_{\alpha \in \Phi_{u_{2}}}\langle\alpha, \tau\rangle .
$$

But by [Kum02, Lemma 1.3.22], $w^{-1} \rho-\rho=\sum_{\alpha \in \Phi_{w}} \alpha$ and the lemma follows.

Because of Lemma 18, Lemma 17 can be restated as: by assigning the degree $\left\langle v^{-1} \rho-\rho, \tau\right\rangle \in \mathrm{Z}$ to $\epsilon_{v}$, one obtains a filtration of the cohomology ring $\mathrm{H}^{*}(G / P, \mathrm{z})$. Then $\odot_{0}$ is defined to be the product of the associated graded ring.

Then $\odot_{0}$ satisfies (with obvious identifications):

$$
\forall u_{1}, u_{2} \in W^{P} \quad \epsilon_{u_{1}} \odot_{0} \epsilon_{u_{2}}=\sum_{v \in W^{P}} \odot_{0}^{0} n_{u_{1} u_{2}}^{v} \epsilon_{v}
$$

where

$$
\begin{aligned}
{ }^{\odot 0} n_{u_{1} u_{2}}^{v} & =0 & \text { if } \delta_{u_{1} u_{2}}^{v} \neq 0 \\
& =n_{u_{1} u_{2}}^{v} & \text { if } \delta_{u_{1} u_{2}}^{v}=0
\end{aligned}
$$

Let $Z(L)$ denote the center of $L$ and $Z(L)^{\circ}$ denote its neutral component. Given an $L$-module and a character $\chi \in X\left(Z(L)^{\circ}\right)$ we denote by $V_{\chi}=\left\{v \in V: \forall t \in Z(L)^{\circ} \quad t v=\chi(t) v\right\}$ the associated weight space. A priori, this construction of $\odot_{0}$ depends on our choice of an element $\tau \in \oplus_{\alpha_{j} \notin \Delta(P)} \mathrm{Z}_{>0} \varpi_{\alpha_{j}^{\vee}}$. Actually, it does not depends on: 
Lemma 19. Let $u_{1}, u_{2}$, and $v$ in $W^{P}$ such that ${ }^{{ }_{0}^{0}} n_{u_{1} u_{2}}^{v} \neq 0$. Then, for any $\chi \in X\left(Z(L)^{\circ}\right)$

$$
\operatorname{dim}\left(\left(\mathcal{T}_{v}\right)_{\chi}\right)=\operatorname{dim}\left(\left(\frac{\mathcal{T}}{\mathcal{T}^{u_{1}}}\right)_{\chi}\right)+\operatorname{dim}\left(\left(\frac{\mathcal{T}}{\mathcal{T}^{u_{2}}}\right)_{\chi}\right) .
$$

Proof. As in the proof of Lemma 17, choose $p_{1}, p_{2}$, and $p_{3}$ in $P$ such that the linear map (15) is an isomorphism. Up to multiplying by $p_{3}^{-1}$ we assume that $p_{3}$ is trivial. Then

$$
\bar{\Theta}: \mathcal{T}_{v} \longrightarrow \frac{\mathcal{T}}{p_{1} \mathcal{T}^{u_{1}}} \oplus \frac{\mathcal{T}}{p_{2} \mathcal{T}^{u_{2}}}
$$

is an isomorphism. For $i=1,2$, write $p_{i}=g_{i}^{u} l_{i}$ with $g_{i}^{u} \in P^{u}$ and $l_{i} \in L$.

Let $k \in \mathrm{Z}$ and $x \in \mathcal{T}_{-k}$. Then

$$
g_{i}^{u} x \in x+\mathcal{T}^{k+1} .
$$

Fix bases of $\mathcal{T}_{v}, \frac{\mathcal{T}}{p_{1} \mathcal{T}^{u_{1}}}$ and $\frac{\mathcal{T}}{p_{2} \mathcal{T}^{u_{2}}}$ adapted to the filtrations. Using (16), one can check that the matrices of $\operatorname{gr} \bar{\Theta}$ and of

$$
\tilde{\Theta}: \mathcal{T}_{v} \longrightarrow \frac{\mathcal{T}}{l_{1} \mathcal{T}^{u_{1}}} \oplus \frac{\mathcal{T}}{l_{2} \mathcal{T}^{u_{2}}}
$$

coincide. Now Lemma 16] shows that $\delta_{u_{1} u_{2}}^{v}=0$ if and only if $\tilde{\Theta}$ is an isomorphism. Since $\tilde{\Theta}$ is $Z(L)^{\circ}$-equivariant, we have

$$
\operatorname{dim}\left(\left(\mathcal{T}_{v}\right)_{\chi}\right)=\operatorname{dim}\left(\left(\frac{\mathcal{T}}{l_{1} \mathcal{T}^{u_{1}}}\right)_{\chi}\right)+\operatorname{dim}\left(\left(\frac{\mathcal{T}}{l_{2} \mathcal{T}^{u_{2}}}\right)_{\chi}\right),
$$

for any $\chi$. Since $Z(L)^{\circ}$ is central in $L$, we have for $i=1,2$

$$
\operatorname{dim}\left(\left(\frac{\mathcal{T}}{l_{i} \mathcal{T}^{u_{i}}}\right)_{\chi}=\operatorname{dim}\left(\left(\frac{\mathcal{T}}{\mathcal{T}^{u_{i}}}\right)_{\chi}\right.\right.
$$

The lemma is proved.

\subsection{On Levi movability.}

Proposition 2. We keep notation and assumptions of Section 7.2 and assume in addition that $P$ is of finite type. Recall that $n_{u_{1} u_{2}}^{v} \neq 0$. We assume that there exist $l_{1}, l_{2}, l \in L$ such that

$$
l_{1} u_{1}^{-1} \stackrel{\circ}{X}^{u_{1}} \cap l_{2} u_{2}^{-1} \stackrel{\circ}{X}^{u_{2}} \cap l v^{-1} \stackrel{\circ}{X}_{v}
$$

is finite. Then

$$
\delta_{u_{1} u_{2}}^{v}=0 .
$$

Proof. Identify $P^{u,-}$ with an $L$-stable open subset of $G / P$. For any $n \geq 2$, consider the normal group $P_{\geq n}^{u,-}$ of $P^{u,-}$ such that $P^{u,-} / P_{\geq n}^{u,-}$ is a finite dimensional unipotent algebraic group with Lie algebra

$$
\bigoplus_{\alpha \in \Phi, 1 \leq\langle\alpha, \tau\rangle<n} \mathfrak{g}_{\alpha}
$$


(see [Kum02, Lemma 6.1.11]). Let $\pi_{n}: P^{u,-} \longrightarrow P^{u,-} / P_{\geq n}^{u,-}$ be the quotient map. Observe that the actions of $\tau$ and $L$ commute and that $P_{\geq n}^{u,-}$ is $L$-stable.

Since $\Phi_{u_{i}}$ and $\Phi_{v}$ are finite, there exists $N$ such that for any $i=1,2$,

$$
u_{i}^{-1} B^{-} u_{i} \cap P \supset P_{\geq N}^{u,-}
$$

and

$$
v^{-1} B v \cap P_{\geq N}^{u,-}=\{e\} .
$$

Then $\pi_{N}\left(u_{i}^{-1} \stackrel{\circ}{X}^{u_{i}}\right)$ has codimension $l\left(u_{i}\right)$, for $i=1,2$; and $\pi_{N}\left(v^{-1} \stackrel{\circ}{X}_{v}\right)$ has dimension $l(v)$. Moreover, $\pi_{N}$ maps bijectively $l_{1} u_{1}^{-1} \dot{\circ}^{u_{1}} \cap l_{2} u_{2}^{-1} \dot{X}^{u_{2}} \cap$ $l v^{-1} \stackrel{\circ}{X}_{v}$ onto

$$
\pi_{N}\left(l_{1} u_{1}^{-1} \stackrel{\circ}{X}^{u_{1}}\right) \cap \pi_{N}\left(l_{2} u_{2}^{-1} \stackrel{\circ}{X}^{u_{2}}\right) \cap \pi_{N}\left(l v^{-1} \stackrel{\circ}{X}_{v}\right) .
$$

Consider now the exponential map

$$
\operatorname{Exp}: \oplus_{\alpha \in \Phi, 1 \leq\langle\alpha, \tau\rangle<n} \mathfrak{g}_{\alpha} \longrightarrow P^{u,-} / P_{\geq N}^{u,-} .
$$

Since $P^{u,-} / P_{\geq N}^{u,-}$ is unipotent, Exp is an isomorphism of varieties. Let $\mathfrak{k}_{1}, \mathfrak{k}_{2}$ and $\mathfrak{k}_{3}$ denote the Lie algebras of $\pi_{N}\left(l_{1} u_{1}^{-1} \dot{\circ}^{u_{1}}=l_{1} u_{1}^{-1} U^{-} u_{1} l_{1}^{-1} \cap\right.$ $\left.P^{u,-}\right), \pi_{N}\left(l_{1} u_{2}^{-1} \stackrel{\circ}{X}^{u_{2}}\right)$ and $\pi_{N}\left(l v^{-1} \stackrel{\circ}{X}_{v}\right)$ respectively. These subspaces are stable by the action of $\tau$ and decompose as $\mathfrak{k}_{i}=\oplus_{n<0} \mathfrak{k}_{i}^{n}$. Since the intersection (17) is finite, $\mathfrak{k}_{1} \cap \mathfrak{k}_{2} \cap \mathfrak{k}_{3}=\{0\}$. Since $\operatorname{dim}\left(\mathfrak{k}_{3}\right)=$ $\operatorname{codim}\left(\mathfrak{k}_{1}\right)+\operatorname{codim}\left(\mathfrak{k}_{2}\right)$, it follows that the natural map

$$
\mathfrak{k}_{3} \longrightarrow \frac{\oplus_{\alpha \in \Phi, 1 \leq\langle\alpha, \tau\rangle<n} \mathfrak{g}_{\alpha}}{\mathfrak{k}_{2}} \oplus \frac{\oplus_{\alpha \in \Phi, 1 \leq\langle\alpha, \tau\rangle<n} \mathfrak{g}_{\alpha}}{\mathfrak{k}_{3}}
$$

is an isomorphism $\tau$-equivariant. Then for any integer $n$ the $\tau$-eigenspace $\mathfrak{k}_{3}^{n}$ has dimension $\operatorname{codim}\left(\mathfrak{k}_{1}^{n}\right)+\operatorname{codim}\left(\mathfrak{k}_{2}^{n}\right)$. Since the actions of $L$ and $\tau$ commute, one deduces that $\delta_{u_{1} u_{2}}^{v}=0$.

\section{Multiplicativity in COHOMOlOGY}

8.1. The multiplicativity. Let $B \subset P \subset Q$ be two standard parabolic subgroups of $G$. Let $L^{P}$ and $L^{Q}$ denote the Levi subgroups of $P$ and $Q$ containing $T$. Then $L^{Q} \cap P$ is a parabolic subgroup of $L^{Q}$ and $Q / P=L^{Q} /\left(L^{Q} \cap P\right)$.

In this section, we study relations between structure constants of $\mathrm{H}^{*}(G / P, \mathrm{z}), \mathrm{H}^{*}(G / Q, \mathrm{z})$ and $\mathrm{H}^{*}(Q / P, \mathrm{z})$. To be more precise, we extend results of [Ric12, Res11] from the classical case to the Kac-Moody case.

Let $W_{Q}^{P}$ be the set of minimal length representative in $W_{Q}$ of the classes $W_{Q} / W_{P}$.

Lemma 20. The map

is bijective.

$$
\begin{aligned}
W^{Q} \times W_{Q}^{P} & \longrightarrow W^{P} \\
(\bar{w}, \tilde{w}) & \longmapsto \bar{w} \tilde{w}
\end{aligned}
$$


Proof. Recall that (see [Kum02, Exercice 1.3.E]) $W^{P}=\{w \in W$ : $\left.w^{-1} \Phi^{-} \cap \Phi^{+}\left(L^{P}\right)=\emptyset\right\}$. We first check that $w=\bar{w} \tilde{w}$ belongs to $W^{P}$; this shows that the map of the lemma is well defined. Write

$$
w^{-1} \Phi^{-} \cap \Phi^{+}\left(L^{P}\right)=w^{-1} \bar{w}\left(\bar{w}^{-1} \Phi^{-} \cap \tilde{w} \Phi^{+}\left(L^{P}\right)\right) .
$$

Note that $\Phi^{+}\left(L^{P}\right) \subset \Phi\left(L^{Q}\right)=\tilde{w} \Phi\left(L^{Q}\right)$ and that $\bar{w}^{-1} \Phi^{-} \cap \Phi\left(L^{Q}\right)=$ $\Phi^{-}\left(L^{Q}\right)$ (since $\left.\bar{w} \in W^{Q}\right)$. Hence

$$
\begin{aligned}
w^{-1} \Phi^{-} \cap \Phi^{+}\left(L^{P}\right) & =\tilde{w}^{-1}\left(\bar{w}^{-1} \Phi^{-} \cap \Phi\left(L^{Q}\right) \cap \tilde{w} \Phi^{+}\left(L^{P}\right)\right) \\
& =\tilde{w}^{-1}\left(\Phi^{-}\left(L^{Q}\right) \cap \tilde{w} \Phi^{+}\left(L^{P}\right)\right) \\
& =\tilde{w}^{-1} \Phi^{-}\left(L^{Q}\right) \cap \Phi^{+}\left(L^{P}\right) .
\end{aligned}
$$

This last intersection is empty since $\tilde{w} \in W_{Q}^{P}$. Then $w \in W^{P}$.

Fix now $w \in W^{P}$. If $w=\bar{w} \tilde{w}$ (with $\bar{w} \in W^{Q}$ and $\tilde{w} \in W_{Q}^{P}$ ) then $w W_{Q}=\bar{w} W_{Q}$ and $\bar{w}$ is necessarily the unique representative of $w W_{Q}$ in $W^{Q}$. Since $\tilde{w}=\bar{w}^{-1} w$, this proves that the map is injective.

Consider now the representative $\bar{w}$ of $w W_{Q}$ in $W^{Q}$ and set $\tilde{w}=\bar{w}^{-1} w$. To prove the surjectivity, it remains to prove that $\tilde{w} \in W_{Q}^{P}$. The equality $\bar{w} W_{Q}=w W_{Q}$ implies that $\tilde{w} \in W_{Q}$. Moreover

$$
\begin{gathered}
\tilde{w}^{-1} \Phi^{-}\left(L^{Q}\right) \cap \Phi^{+}\left(L^{P}\right)=w^{-1} \bar{w} \Phi^{-}\left(L^{Q}\right) \cap \Phi^{+}\left(L^{P}\right) \\
\subset w^{-1} \Phi^{-} \cap \Phi^{+}\left(L^{P}\right),
\end{gathered}
$$

since $\bar{w} \Phi^{-}\left(L^{Q}\right) \subset \Phi^{-}\left(\bar{w} \in W^{Q}\right)$. This last intersection is empty since $w \in W^{P}$. The lemma is proved.

Recall that, for $w \in W$

$$
\begin{array}{ll}
X_{w}^{G / P}=\overline{B w P / P} & \stackrel{\circ}{w}_{w}^{G / P}=B w P / P \\
X_{G / P}^{w}=\overline{B^{-} w P / P} & \dot{\circ}_{G / P}^{w}=B^{-} w P / P
\end{array}
$$

Lemma 21. (See [BK06, Lemma 1]) Let $w \in W^{P}$ and $g \in G$.

(i) If $g \dot{X}_{w}^{G / P}$ contains $P / P$ then there exists $p \in P$ such that $g \stackrel{\circ}{X}_{w}^{G / P}=p w^{-1} \stackrel{\circ}{X}_{w}^{G / P}$.

(ii) If $g \dot{\circ}_{G / P}^{w}$ contains $P / P$ then there exists $p \in P$ such that $g \stackrel{\circ}{X}_{G / P}^{w}=p w^{-1} \stackrel{\circ}{X}_{G / P}^{w}$.

Proof. Fix a representative $\dot{w}$ of $w$ in $N(T)$. Let $b \in B$ and $p \in P$ such that $g b \dot{w}=p$. Then $g \stackrel{\circ}{X}_{w}^{G / P}=p \dot{w}^{-1} b^{-1} \stackrel{\circ}{X}_{w}^{G / P}=p w^{-1} \stackrel{\circ}{X}_{w}^{G / P}$. The second assertion works similarly.

Lemma 22. Let $\tilde{w} \in W_{Q}^{P}$ and $\bar{w} \in W^{Q}$. Set $w=\bar{w} \tilde{w}$. Then

(i) $\bar{w}^{-1} \stackrel{\circ}{X}_{w}^{G / P} \cap Q / P=\stackrel{\circ}{X}_{\tilde{w}}^{Q / P}$;

(ii) $\bar{w}^{-1} \stackrel{\circ}{X}_{G / P}^{w} \cap Q / P=\stackrel{\circ}{X} \underset{Q / P}{\tilde{w}}$. 
Proof. Note that $\bar{w}^{-1} \stackrel{\circ}{X}_{w}^{G / P} \cap Q / P$ is stable by the action of $\bar{w}^{-1} B \bar{w} \cap Q$. Since $\bar{w} \in W^{Q}, \bar{w}^{-1} B \bar{w} \cap Q$ contains $L^{Q} \cap B$. Moreover each $\left(L^{Q} \cap B\right)$ orbit in $Q / P$ contains a $T$-fixed point. Hence

$$
\bar{w}^{-1} \stackrel{\circ}{X}_{w}^{G / P} \cap Q / P=\bigcup_{x \in\left(\bar{w}^{-1} \dot{\varkappa}_{w}^{G / P} \cap Q / P\right)^{T}}\left(L^{Q} \cap B\right) . x .
$$

But $\left(\bar{w}^{-1} \stackrel{\circ}{X}_{w}^{G / P} \cap Q / P\right)^{T} \subset \bar{w}^{-1}\left(\stackrel{\circ}{X}_{w}^{G / P}\right)^{T}=\{\tilde{w} P / P\}$. The first assertion of the lemma follows. The second one works similarly.

We can now prove the main result of this section.

Proposition 3. Let $u_{1}, u_{2}$, and $v$ in $W^{P}$. Write $u_{1}=\bar{u}_{1} \tilde{u}_{1}, u_{2}=\bar{u}_{2} \tilde{u}_{2}$, and $v=\bar{v} \tilde{v}$ as in Lemma 20. We assume that $l(v)=l\left(u_{1}\right)+l\left(u_{2}\right)$ and $l(\bar{v})=l\left(\bar{u}_{1}\right)+l\left(\bar{u}_{2}\right)$.

Consider the structure constants $n_{u_{1} u_{2}}^{v}, n_{\bar{u}_{1} \bar{u}_{2}}^{\bar{v}}$, and $n_{\tilde{u}_{1} \tilde{u}_{2}}^{\tilde{v}}$ in $\mathrm{H}^{*}(G / P, \mathrm{z})$, $\mathrm{H}^{*}(G / Q, \mathrm{z})$, and $\mathrm{H}^{*}(Q / P, \mathrm{z})$ respectively.

Then

$$
n_{u_{1} u_{2}}^{v}=n_{\bar{u}_{1} \bar{u}_{2}}^{\bar{v}} n_{\tilde{u}_{1} \tilde{u}_{2}}^{\tilde{v}} .
$$

Proof. Since $B$ is irreducible, Lemma 6 implies that there exists $b \in B$ such that

(i) $X_{G / P}^{u_{1}} \cap b X_{v}^{u_{2}}=\stackrel{\circ}{X}_{G / P}^{u_{1}} \cap \stackrel{\circ}{X}_{v}^{G / P} \cap b \stackrel{\circ}{X}_{G / P}^{u_{2}}$ is transverse, and

(ii) $X_{G / Q}^{\bar{u}_{1}} \cap b X_{\bar{v}}^{\bar{u}_{2}}=\stackrel{\circ}{X}_{G / Q}^{\bar{u}_{1}} \cap \stackrel{\circ}{X}_{\bar{v}}^{G / Q} \cap b \dot{X}_{G / Q}^{\bar{u}_{2}}$ is transverse.

By Lemma 7, it remains to determine the cardinality of the intersection (i). We do this by counting in each fiber of the $G$-equivariant projection $\pi: G / P \longrightarrow G / Q$.

Fix $g \in G$ such that $g Q / Q \in X_{G / Q}^{\bar{u}_{1}} \cap b X_{\bar{v}}^{\bar{u}_{2}}$. Then $Q / Q \in g^{-1} \stackrel{\circ}{X}_{G / Q}^{\bar{u}_{1}} \cap$ $g^{-1} \stackrel{\circ}{X}_{\bar{v}}^{G / Q} \cap g^{-1} b \dot{X}_{G / Q}^{\bar{u}_{2}}$. As in Lemma 21, there exist $q_{1}, q_{2}$, and $q$ in $Q$ such that $g^{-1} \in q \dot{\bar{v}}^{-1} B, g^{-1} \in q_{1} \dot{\bar{u}}_{1}^{-1} B^{-}$, and $g^{-1} b \in q_{2} \dot{\bar{u}}_{2}^{-1} B^{-}$. Let $l_{1}$, $l_{2}$, and $l$ in $Q$ such that $q_{1} l_{1}^{-1}, q_{2} l_{2}^{-1}$, and $q l^{-1}$ belong to $Q^{u}$. Then

$$
\begin{aligned}
I & :=g^{-1}\left(\stackrel{\circ}{X}_{G / P}^{u_{1}} \cap b \stackrel{\circ}{X}_{G / P}^{u_{2}} \cap \stackrel{\circ}{X}_{v}^{G / P} \cap \pi^{-1}(g Q / Q)\right) \\
& =q_{1} \bar{u}_{1}^{-1} \stackrel{\circ}{X}_{G / P}^{u_{1}} \cap q_{2} \bar{u}_{2}^{-1} \stackrel{\circ}{X}_{G / P}^{u_{2}} \cap q \bar{v}^{-1} \stackrel{\circ}{X}_{v}^{G / P} \cap Q / P \\
& =q \stackrel{\circ}{X}_{\tilde{v}}^{Q / P} \cap q_{1} \stackrel{\circ}{X}_{Q / P}^{\tilde{u}_{1}} \cap q_{2} \stackrel{\circ}{X}_{Q / P}^{\tilde{u}_{2}} \\
& =l \stackrel{\circ}{X}_{\tilde{v}}^{Q / P} \cap l_{1} \stackrel{\circ}{X}_{Q / P}^{\tilde{u}_{1}} \cap l_{2} \stackrel{\circ}{X}_{Q / P}^{\tilde{u}_{2}}
\end{aligned}
$$

The last equality holds since $Q^{u}$ acts trivially on $Q / P$. Moreover, since $X_{G / P}^{u_{1}} \cap b X_{v}^{u_{2}}=\stackrel{\circ}{X}_{G / P}^{u_{1}} \cap \stackrel{\circ}{X}_{v}^{G / P} \cap b \dot{X}_{G / P}^{u_{2}}$, we also have

$$
I=l X_{\tilde{v}}^{Q / P} \cap l_{1} X_{Q / P}^{\tilde{u}_{1}} \cap l_{2} X_{Q / P}^{\tilde{u}_{2}} .
$$




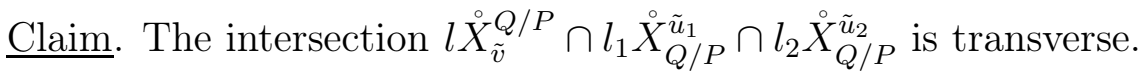

Let $x$ be a point in this intersection. By Lemma 6 , the map

$$
T_{x} g^{-1} \stackrel{\circ}{X}_{v}^{G / P} \longrightarrow \frac{T_{x} G / P}{T_{x} g^{-1} \stackrel{\circ}{X}_{G / P}^{u_{1}}} \oplus \frac{T_{x} G / P}{T_{x} g^{-1} b \dot{X}_{G / P}^{u_{2}}}
$$

is an isomorphism. Hence, the natural map

$$
T_{x} g^{-1} \stackrel{\circ}{X}_{v}^{G / P} \cap T_{x} Q / P \longrightarrow \frac{T_{x} Q / P}{T_{x} g^{-1} \stackrel{o}{X}_{G / P}^{u_{1}} \cap T_{x} Q / P} \oplus \frac{T_{x} Q / P}{T_{x} g^{-1} b \dot{X}_{G / P}^{u_{2}} \cap T_{x} Q / P}
$$

is injective. Since $T_{x} l \stackrel{\circ}{X}_{\tilde{v}}^{Q / P} \subset T_{x} g^{-1} \stackrel{\circ}{X}_{v}^{G / P} \cap T_{x} Q / P$ (and similar inclusions hold for $u_{1}$ and $u_{2}$ ), we deduce that the natural map

$$
T_{x} l \stackrel{\circ}{X}_{\tilde{v}}^{Q / P} \longrightarrow \frac{T_{x} Q / P}{T_{x} l_{1} \dot{O}_{Q / P}^{\tilde{u}_{1}}} \oplus \frac{T_{x} Q / P}{T_{x} l_{2} \dot{O}_{Q / P}^{\tilde{u}_{2}}}
$$

is injective. The assumption on the length of elements of $W_{Q}^{P}$ implies that it is in fact an isomorphism. The claim is proved.

The claim and Lemma 7 imply that the cardinal of $I$ is $n_{\tilde{u}_{1}}^{\tilde{u}} \tilde{u}_{2}$. Since this holds for any of the $n_{\bar{u}_{1} \bar{u}_{2}}^{\bar{u}_{2}}$ points in $X_{G / Q}^{\bar{u}_{1}} \cap b X_{\bar{v}}^{\bar{u}_{2}}$, we get that $X_{G / P}^{u_{1}} \cap b X_{v}^{u_{2}}$ has cardinality $n_{\tilde{u}_{1} \tilde{u}_{2}}^{\tilde{v}} n_{\bar{u}_{1} \bar{u}_{2}}^{\bar{v}}$. We conclude by applying Lemma 7 in $G / P$.

8.2. Application to the BKB-product. The following lemma allows to apply Proposition 3 to any structure constants of the BKBproduct.

Lemma 23. Let $P \subset Q$ be two standard parabolic subgroups of $G$. Let $u_{1}, u_{2}$, and $v$ in $W^{P}$ such that ${ }^{\odot}{ }^{\circ} n_{u_{1} u_{2}}^{v} \neq 0$.

Then $l(\bar{v})=l\left(\bar{u}_{1}\right)+l\left(\bar{u}_{2}\right)$.

Proof. Let $Q^{-}$be the opposite subgroup of $Q$ and $Q^{u,-}$ its "unipotent" subgroup. Let $\mathfrak{q}^{u,-}$ be the Lie algebra of $Q^{u,-}$. Note that

$$
l(\bar{v})=\operatorname{dim}\left(\mathcal{T}_{v} \cap \mathfrak{q}^{u,-}\right) \text { and } l\left(\bar{u}_{i}\right)=\operatorname{dim}\left(\frac{\mathfrak{q}^{u,-}}{\mathcal{T}^{u_{i}} \cap \mathfrak{q}^{u,-}}\right) \forall i=1,2 .
$$

There exists a one parameter subgroup $\tau_{Q}$ of $Z\left(L^{Q}\right)$ such that $\mathfrak{q}^{u,-}$ is the sum of the negative weight spaces for $\tau_{Q}$. Since $Z\left(L^{Q}\right)^{\circ}$ is contained in $Z\left(L^{P}\right)^{\circ}$ :

$$
\mathcal{T}_{w} \cap \mathfrak{q}^{u,-}=\bigoplus_{\chi \in X\left(Z\left(L^{P}\right)\right)^{\circ}\left\langle\chi, \tau_{Q}\right\rangle<0}\left(\mathcal{T}_{w}\right)_{\chi}
$$

for any $w \in W$. Now the equality of the lemma is a direct consequence of Lemma 19 . 


\section{The Untwisted AFFine CASE}

9.1. Notation. Let $\dot{\mathfrak{g}}$ be a complex finite dimensional simple Lie algebra with Cartan subalgebra $\dot{\mathfrak{h}}$ and Borel subalgebra $\dot{\mathfrak{b}} \supset \dot{\mathfrak{h}}$. Let $\dot{\alpha}_{1}, \ldots, \dot{\alpha}_{l}$ denote the simple roots, $\dot{\alpha}_{1}^{\vee}, \ldots, \dot{\alpha}_{l}^{\vee}$ the simple coroots, $\dot{\theta}$ the highest root and $\dot{\theta}^{\vee}$ the highest coroot. For any simple root $\dot{\alpha}$, we denote by $\varpi_{\dot{\alpha}}$ the corresponding fundamental weight and by $\varpi_{\dot{\alpha}} \vee$ the corresponding fundamental coweight. Let $\dot{P}_{+}$denote the set of dominant integral weights for $\dot{\mathfrak{g}}$. Set $\dot{\rho}=\sum_{i=1}^{l} \varpi_{\dot{\alpha}_{i}}$.

Endow $\mathfrak{g}=\dot{\mathfrak{g}} \otimes \mathrm{C}\left[z, z^{-1}\right] \oplus \mathrm{C} c \oplus \mathrm{C} d$ with the usual Lie bracket (see e.g. Kum02, Chap XIII]). Set $\mathfrak{h}=\dot{\mathfrak{h}} \oplus \mathrm{C} c \oplus \mathrm{C} d$. Define $\Lambda$ and $\delta$ in $\mathfrak{h}^{*}$ by

$$
\begin{aligned}
& \delta: \dot{\mathfrak{h}} \longmapsto 0, c \longmapsto 0, d \longmapsto 1 ; \\
& \Lambda: \dot{\mathfrak{h}} \longmapsto 0, c \longmapsto 1, d \longmapsto 0 .
\end{aligned}
$$

We identify $\dot{\mathfrak{h}}^{*}$ with the orthogonal of $\mathrm{C} c \oplus \mathrm{C} d$ in $\mathfrak{h}^{*}$ in such a way that $\mathfrak{h}^{*}=\dot{\mathfrak{h}}^{*} \oplus \mathrm{C} \Lambda \oplus \mathrm{C} \delta$. The simple roots of $\mathfrak{g}$ are

$$
\alpha_{0}=\delta-\dot{\theta}, \dot{\alpha}_{1}, \ldots, \dot{\alpha}_{l}
$$

The simple coroots of $\mathfrak{g}$ are

$$
\alpha_{0}^{\vee}=c-\dot{\theta}^{\vee}, \dot{\alpha}_{1}^{\vee}, \ldots, \dot{\alpha}_{l}^{\vee} .
$$

For any simple root $\dot{\alpha}_{i}$ of $\dot{\mathfrak{g}}$, set $\varpi_{\alpha_{i}}=\varpi_{\dot{\alpha}_{i}}+\varpi_{\dot{\alpha}_{i}}\left(\dot{\theta}^{\vee}\right) \Lambda \in \mathfrak{h}^{*}$. Set $\varpi_{\alpha_{0}}=\Lambda$. A choice of fundamental weights for $\mathfrak{g}$ is $\varpi_{\alpha_{0}}, \ldots, \varpi_{\alpha_{l}}$. In particular

$$
\rho=\dot{\rho}+h^{\vee} \Lambda
$$

where $h^{\vee}=1+\left\langle\dot{\rho}, \dot{\theta}^{\vee}\right\rangle$ is the dual Coxeter number. Set

$$
\mathfrak{h}_{\mathrm{z}}^{*}=\mathrm{Z} \varpi_{\alpha_{0}} \oplus \cdots \oplus \mathrm{Z} \varpi_{\alpha_{l}} \oplus \mathrm{Z} \delta,
$$

and

$$
\begin{aligned}
P_{+} & =\mathrm{z}_{\geq 0} \varpi_{\alpha_{0}} \oplus \cdots \oplus \mathrm{z}_{\geq 0} \varpi_{\alpha_{l}} \oplus \mathrm{z} \delta, \\
& =\left\{\dot{\lambda}+\ell \Lambda+b \delta: \dot{\lambda} \in \dot{P}_{+} \text {and }\left\langle\dot{\lambda}, \dot{\theta}^{\vee}\right\rangle \leq \ell\right\} .
\end{aligned}
$$

Denote by $P_{++}=\mathrm{z}_{>0} \varpi_{\alpha_{0}} \oplus \cdots \oplus \mathrm{z}_{>0} \varpi_{\alpha_{l}} \oplus \mathrm{z} \delta$, the set of regular dominant weights. The chosen fundamental coweights are

$$
\varpi_{\alpha_{0}^{\vee}}=d \quad \varpi_{\alpha_{i}^{\vee}}=\varpi_{\dot{\alpha}_{i}^{\vee}}+\left\langle\varpi_{\dot{\alpha}_{i}^{\vee}}, \dot{\theta}\right\rangle d .
$$

Set $\dot{Q}^{\vee}=\oplus_{i=1}^{l} \mathrm{z} \dot{\alpha}_{i}^{\vee}$. Then $W=\dot{Q}^{\vee} . \dot{W}$. Moreover $h \in \dot{Q}^{\vee}$ acts on $\mathfrak{h}$ by

$$
h \cdot(x+k d+\ell c)=x+k h+k d+\left(\ell-(x, h)-k \frac{(h, h)}{2}\right) c .
$$


9.2. Essential inequalities and BKB-product. We are now interested in the inequlities (8) of Proposition 11 that are equalities for some regular elements of $\Gamma(\mathfrak{g})$. We prove that such inequalities necessarily appear in Theorem 1 .

Theorem 6. We use notation of Proposition 1 and assume that $n_{u_{1}, u_{2}}^{v}=$ 1. Let $\tau \in \oplus_{\alpha_{j} \notin \Delta(P)} \mathrm{Z}_{>0} \varpi_{\alpha_{j}^{\vee}}$. Let $\left(\lambda_{1}, \lambda_{2}, \mu\right) \in\left(P_{+}\right)^{3}$ such that

$$
\left\langle\lambda_{1}, u_{1} \tau\right\rangle+\left\langle\lambda_{2}, u_{2} \tau\right\rangle=\langle\mu, v \tau\rangle .
$$

Assume that $\mu$ is regular and that

$$
\exists N>0 \quad L(N \mu) \subset L\left(N \lambda_{1}\right) \otimes L\left(N \lambda_{2}\right) .
$$

Then, $\epsilon_{v}$ appears with multiplicity 1 in $\epsilon_{u_{1}} \odot_{0} \epsilon_{u_{2}}$.

Consider the line bundle $\mathcal{L}=\mathcal{L}_{-}\left(\lambda_{1}\right) \otimes \mathcal{L}_{-}\left(\lambda_{2}\right) \otimes \mathcal{L}(\mu)$ on $\mathrm{X}$, and the closed subset $C=L u_{1}^{-1} \underline{O}^{-} \times L u_{2}^{-1} \underline{O}^{-} \times L v^{-1} \underline{O}$ of $\mathrm{X}$.

For $i=1,2$, consider the maximal parabolic subgroup $Q_{i}$ containing $B^{-}$such that $\lambda_{i}$ extends to $Q_{i}$. Set $\underline{\mathrm{x}}=G / Q_{1} \times G / Q_{2} \times G / B$ and $\pi: \mathrm{X} \longrightarrow \underline{\mathrm{X}}$. Set $\underline{C}=\pi(C)$ and $\underline{C}^{+}=\pi\left(C^{+}\right)$. Let $\underline{\mathcal{L}}$ be the $G$ linearized line bundle on $\underline{\mathrm{X}}$ such that $\pi^{*}(\underline{\mathcal{L}})=\mathcal{L}$.

In this paper, we denote by $C^{\mathrm{ss}}\left(\mathcal{L}_{\mid C}, L\right)$ the set of points $x \in C$ such that there exists a $L$-invariant section $\sigma$ of some positive power $\mathcal{L}_{\mid C}^{\otimes N}$ of $\mathcal{L}_{\mid C}$ such that $\sigma(x) \neq 0$. Note that this definition is the standard one (see [MFK94]) only if $\mathcal{L}_{\mid C}$ is ample. Moreover, $C^{\text {ss }}\left(\mathcal{L}_{\mid C}, L\right)=C \cap$ $\pi^{-1}\left(\underline{C}^{\mathrm{ss}}\left(\underline{\mathcal{L}}_{\mid C}, L\right)\right)$.

The following lemma is a consequence of Theorem 5. We include here a more direct proof.

Lemma 24. With the assumptions of Theorem [6, the set $C^{\mathrm{ss}}\left(\mathcal{L}_{\mid C}, L\right)$ is not empty. In particular, $Z(L)^{\circ}$ acts trivially on $\mathcal{L}_{\mid C}$.

Proof. By Lemma 4, there exists a $G$-invariant section $\sigma \in \mathrm{H}^{0}\left(\mathrm{x}, \mathcal{L}^{\otimes N}\right)^{G}$. By Lemma 10, the image of $\eta: G \times_{P} C^{+} \longrightarrow \mathrm{X}$ contains a nonempty open subset of $\mathrm{X}$. Since $\mathrm{X}$ is irreducible and $G$-invariant, we deduce that there exists $x \in C^{+}$such that $\sigma(x) \neq 0$.

Set $y=\lim _{t \rightarrow 0} \tau(t) x$. Consider the map $\theta_{x}$ defined in the proof of Lemma 14. Since $\mu^{\mathcal{L}}(C, \tau)=0, \theta_{x}^{*}(\mathcal{L})$ is trivial as a $C^{*}$-linearized line bundle. We deduce that $\tilde{y}:=\lim _{t \rightarrow 0} \tau(t) \sigma(x)$ exists and belongs to $\mathcal{L}_{y}-\{y\}$. But, $\sigma$ being $G$-invariant, $\tilde{y}=\sigma(y)$. In particular $\tilde{y}$ belongs to $C^{\mathrm{ss}}\left(\mathcal{L}_{\mid C}, L\right)$.

Since $Z(L)$ acts trivially on $C$ and $\sigma$ is $G$-invariant, it fixes $\tilde{y}$. Then $Z(L)$ acts trivially on $\mathcal{L}_{\mid C}^{\otimes N}$ and $Z(L)^{\circ}$ acts trivially on $\mathcal{L}_{\mid C}$.

We now prove a lemma on the points of $\underline{C}^{\mathrm{ss}}\left(\underline{\mathcal{L}}_{\mid \underline{C}}, L\right)$.

Lemma 25. Let $x \in \underline{C}^{\mathrm{ss}}\left(\underline{\mathcal{L}}_{\mid \underline{C}}, L\right)$. Then $G_{x} \cap P^{u,-}$ is trivial. 
Proof. Fix $\tilde{x} \in C$ such that $\pi(\tilde{x})=x$. By Theorem 5, one can find a $G$-invariant section $\sigma$ of some positive power $\mathcal{L}^{\otimes N}$ of $\mathcal{L}$ such that $\sigma(\tilde{x}) \neq 0$. For any $\left(w_{1}, w_{2}, w_{3}\right) \in W, H^{0}\left(\pi\left(X_{w_{1}}^{G / B^{-}} \times X_{w_{3}}^{G / B}\right), \underline{\mathcal{L}}\right)$ is isomorphic to $H^{0}\left(X_{w_{1}}^{G / B^{-}} \times X_{w_{3}}^{G / B}, \mathcal{L}\right)$. In particular, $\sigma$ descends to a $G$-invariant section $\underline{\sigma}$ of $\underline{\mathcal{L}}$ on $\underline{\mathrm{X}}$. The set $\underline{\mathrm{X}}_{\sigma}=\{y \in \underline{\mathrm{X}}: \underline{\sigma}(y) \neq 0\}$ is a $G$-stable affine ind-variety containing $x$.

Write $x=\left(l_{1} u_{1}^{-1} Q_{1} / Q_{1}, l_{2} u_{2}^{-1} Q_{2} / Q_{2}, l v^{-1} \underline{o}\right)$, with $l_{1}, l_{2}$ and $l$ in $L$. Then $G_{x} \cap P^{u,-}$ is contained in $l\left(v^{-1} B v \cap P^{u,-}\right) l^{-1}$. By [Kum02, Example 6.1.5.b], $v^{-1} B v \cap P^{u,-}$ is a finite dimensional unipotent group. In particular, $G_{x} \cap P^{u,-}$ is connected and it is sufficient to prove that its Lie algebra is trivial.

Assume that there exists a nonzero vector $\xi \in \operatorname{Lie}\left(G_{x} \cap P^{u,-}\right)$. Consider a morphism

$$
\phi: \mathrm{SL}_{2}(\mathrm{C}) \longrightarrow G
$$

such that $T_{1} \phi(E)=\xi$, given by Proposition 11.1. Look the induced $\mathrm{SL}_{2}(\mathrm{C})$-action on $\underline{\mathrm{x}}$. The unipotent subgroup $U_{2}=\left(\begin{array}{ll}1 & * \\ 0 & 1\end{array}\right)$ of $\mathrm{SL}_{2}(\mathrm{C})$ fixes the point $x$. Since $\mathrm{SL}_{2}(\mathrm{C}) / U_{2} \simeq \mathrm{C}^{2}-\{(0,0)\}$, one gets a regular map

$$
\bar{\phi}: \mathrm{C}^{2}-\{(0,0)\} \longrightarrow \underline{\mathrm{X}} .
$$

Since $\underline{x}_{\sigma}$ is $G$-stable, the image of $\bar{\phi}$ is contained in $\underline{x}_{\sigma}$. Since $\underline{x}_{\sigma}$ is an affine ind-variety, Arthog's lemma implies that $\bar{\phi}$ extends to a regular map

$$
\tilde{\phi}: \mathrm{C}^{2} \longrightarrow \underline{\mathrm{x}}
$$

By density, $\tilde{\phi}$ is $\mathrm{SL}_{2}(\mathrm{C})$-equivariant. In particular the point $\tilde{\phi}(0,0)$ is fixed by $\mathrm{SL}_{2}(\mathrm{C})$. This is a contradiction, since $v^{-1} \mathfrak{b} v$ contains no copy of $\mathfrak{s l}_{2}(\mathrm{C})$.

Proof of Theorem 6. Consider the group $X(T)^{Z(L)^{\circ}}$ of characters $\chi$ of $T$ such that $\chi_{\mid Z(L)}{ }^{\circ}$ is trivial. By Lemma 24, $\mathcal{L}_{-}\left(\lambda_{1}\right) \otimes \mathcal{L}_{-}\left(\lambda_{2}\right) \otimes \mathcal{L}(\mu)$ belongs to $\operatorname{Pic}^{L / Z(L)^{\circ}}(\underline{C})$. Set

$$
\begin{aligned}
\gamma: X(T)^{Z(L)^{\circ} \otimes \mathrm{Q}} & \longrightarrow \operatorname{Pic}^{L / Z(L)^{\circ}}(\underline{C}) \otimes \mathrm{Q} \\
\mu^{\prime} & \longmapsto\left(\mathcal{L}_{-}\left(\lambda_{1}\right) \otimes \mathcal{L}_{-}\left(\lambda_{2}\right) \otimes \mathcal{L}\left(\mu+\mu^{\prime}\right)\right)_{\mid \underline{C}} .
\end{aligned}
$$

The set of $\mu^{\prime}$ such that some positive power of $\gamma\left(\mu^{\prime}\right)$ is ample and $\underline{C}^{\text {ss }}\left(\gamma\left(\mu^{\prime}\right), L / Z(L)^{\circ}\right)$ is not empty is a convex set denoted $\mathcal{C}^{L}(\underline{C})$. But, the image of $\gamma$ is abundant in the sense of [DH98, Section 4.1]. As a consequence, for $\mu^{\prime}$ general in $\mathcal{C}^{L}(\underline{C})$, there exist stable points in $\underline{C}$ for $\gamma\left(\mu^{\prime}\right)$ and the action of $L / Z(L)^{\circ}$. Fix such a $\mu^{\prime}$ and $N^{\prime}$ such that $N^{\prime} \mu^{\prime} \in X(T)$. Then, $\left(N^{\prime} \lambda_{1}, N^{\prime} \lambda_{2}, N^{\prime}\left(\mu+\mu^{\prime}\right)\right)$ still satisfies equality (20). Moreover, Theorem 5 and the descent argument at the beginning of the proof of Lemma 25 show that it also satisfies condition (21) for some $N$. Then, to prove the theorem, one may assume that $\underline{C}^{\mathrm{s}}\left(\underline{\mathcal{L}}, L / Z(L)^{\circ}\right)$ is not empty. 
The graded algebra $\oplus_{k} \mathrm{H}^{0}\left(\underline{C}, \underline{\mathcal{L}}_{\mid \underline{C}}^{\otimes k}\right)^{L}$ is finitely generated. Fix $d>0$ such that $\mathrm{H}^{0}\left(\underline{C}, \underline{\mathcal{L}}_{\mid \underline{C}}^{\otimes d}\right)^{L}$ generates $\oplus_{k} \mathrm{H}^{0}\left(\underline{C}, \underline{\mathcal{L}}_{\mid \underline{C}}^{\otimes d k}\right)^{L}$. Let $\sigma_{0}, \ldots, \sigma_{N}$ be a C-basis of $\mathrm{H}^{0}\left(\underline{C}, \underline{\mathcal{L}}_{\mid \underline{C}}^{\otimes d}\right)^{L}$. By Theorem $\left[5\right.$, for any $i, \sigma_{i}$ extends to a $G$-invariant section $\tilde{\sigma}_{i}$ on $\underline{\mathrm{X}}$. Set

$$
\underline{\mathrm{X}}^{\mathrm{ss}}(\mathcal{L}):=\left\{x \in \underline{\mathrm{X}}: \exists i \quad \tilde{\sigma}_{i}(x) \neq 0\right\}
$$

and

$$
\begin{aligned}
\pi: \underline{\mathrm{X}}^{\mathrm{ss}}(\underline{\mathcal{L}}) & \longrightarrow \mathrm{CP}^{N} \\
x & \longmapsto\left[\tilde{\sigma}_{0}(x): \cdots: \tilde{\sigma}_{N}(x)\right] .
\end{aligned}
$$

Consider now $\underline{\eta}: G \times_{P} \underline{C}^{+} \longrightarrow \underline{\mathrm{x}}$. Let $\underline{C}^{+ \text {,ss }}(\underline{\mathcal{L}}, L)$ denote the set of points $y \in \underline{C}^{+}$such that $\lim _{t \rightarrow 0} \tau(t) y \in \underline{C}^{\mathrm{ss}}(\underline{\mathcal{L}}, L)$. Since the $\tilde{\sigma}_{i}$ are $G$-invariant, $\eta\left(G \times{ }_{P} \underline{C}^{+, \mathrm{ss}}(\underline{\mathcal{L}}, L)\right)$ is contained in $\mathrm{X}^{\mathrm{ss}}(\mathcal{L})$. By the proof of Proposition 1, this set is dense in $\underline{x}^{\text {ss }}(\underline{\mathcal{L}})$, and hence in $\underline{\mathrm{x}}$ by irreducibility. But $\pi(\underline{\eta}((g P / P, x)))=\pi\left(g^{-1} x\right)$ for any $g \in G, x \in$ $\underline{\mathrm{x}}^{\mathrm{ss}}(\underline{\mathcal{L}}, L)$ such that $(g \bar{P} / P, x) \in G \times{ }_{P} \underline{C}$. Then $\pi \circ \underline{\eta}\left(G \times{ }_{P} \underline{C}^{+, \mathrm{ss}}(\underline{\mathcal{L}}), L\right)$ is contained in $\pi\left(\underline{C}^{\mathrm{ss}}(\underline{\mathcal{L}}, L)\right)=\underline{C}^{\mathrm{ss}}(\underline{\mathcal{L}}, L) / / L$. Since $\underline{C}^{\mathrm{ss}}(\underline{\mathcal{L}}, L) / / L$ is projective, this implies that

$$
\pi\left(\underline{\mathrm{x}}^{\mathrm{ss}}(\underline{\mathcal{L}})\right)=\underline{C}^{\mathrm{ss}}(\underline{\mathcal{L}}, L) / / L:=\operatorname{Proj}\left(\oplus_{k} \mathrm{H}^{0}\left(\underline{C}, \underline{\mathcal{L}}_{\mid \underline{C}}^{\otimes k}\right)^{L}\right) .
$$

Let us fix $\xi \in \underline{C}^{\mathrm{ss}}(\underline{\mathcal{L}}) / / L$ general. Since $\underline{C}^{s}\left(\underline{\mathcal{L}}_{\mid \underline{C}}, L / Z(L)\right)$ is not empty, $\pi^{-1}(\xi) \cap \underline{C}$ is an $L$-orbit L. $x_{0}$. Since $L .\left(u_{2}^{-1} Q_{2} / Q_{2}, v^{-1} \underline{o}\right)$ is open in $L u_{2}^{-1} Q_{2} / Q_{2} \times L v^{-1} \underline{o}$, one may assume that $x_{0}=\left(l_{1} u_{1}^{-1} Q_{1} / Q_{1}, u_{2}^{-1} Q_{2} / Q_{2}, v^{-1} \underline{o}\right)$, for some $l_{1} \in L$. Let $i$ such that $\sigma_{i}\left(x_{0}\right) \neq 0$.

We claim that $\underline{\eta}^{-1}\left(x_{0}\right)$ is finite. Let $g \in G$ such that $\left(g P / P, x_{0}\right) \in$ $\underline{\eta}^{-1}\left(x_{0}\right)$. Set $y=g^{-1} x_{0} \in \underline{C}^{+}$and $z=\lim _{t \rightarrow 0} \tau(t) y$. Consider $\tilde{y}:=$ $\overline{\tilde{\sigma}}_{i}(y)=g^{-1} \tilde{\sigma}_{i}\left(x_{0}\right) \in\left(\underline{\mathcal{L}}^{\otimes d}\right)_{y}-\{y\}$. Since $\mu^{\mathcal{L}}(C, \tau)=0, \tilde{z}:=\lim _{t \rightarrow 0} \tau(t) \tilde{y} \in$ $\left(\mathcal{L}^{\otimes d}\right)_{z}-\{z\}$. But $\tilde{\sigma}_{i}$ being $G$-invariant, $\tilde{\sigma}_{i}(z)=\tilde{z}$. Hence $z \in$ $\underline{\mathrm{x}}^{\mathrm{ss}}(\underline{\mathcal{L}}) \cap \underline{C}=\underline{C}^{\mathrm{ss}}(\mathcal{L})$.

Moreover, $\pi$ being $G$-invariant, we have $\pi(z)=\pi\left(x_{0}\right)$. Hence $z \in$ L. $x_{0}$. Then Lemma 11.3 implies that $y=g^{-1} x_{0} \in P x_{0}$. Hence $g \in$ $G_{x_{0}} P$.

Note that $G_{x_{0}} \subset u_{2}^{-1} Q_{2} u_{2} \cap v^{-1} B v$ is finite dimensional. Moreover, it contains $\tau\left(\mathrm{C}^{*}\right)$. Then, the Lie algebra of $G_{x_{0}}$ decomposes as $\operatorname{Lie}\left(G_{x_{0}}\right)=$ $\left(\operatorname{Lie}\left(G_{x_{0}}\right) \cap \operatorname{Lie}\left(P^{u,-}\right) \oplus\left(\operatorname{Lie}\left(G_{x_{0}}\right) \cap \operatorname{Lie}(P)\right)\right.$. Using Lemma 25, we deduce that the neutral component $G_{x_{0}}^{\circ}$ of $G_{x_{0}}$ is contained in $P$. Then $G_{x_{0}} P / P$ is finite and the claim is proved.

Consider now $\eta: G \times{ }_{P} C^{+} \longrightarrow \mathrm{X}$. Let $\tilde{x}_{0} \in C$ such that $\pi\left(\tilde{x}_{0}\right)=x_{0}$. Since $\pi^{-1}\left(\underline{C}^{+}\right)=C^{+}$, the claim implies that $\eta^{-1}\left(\tilde{x}_{0}\right)$ is finite. With Lemma 9, the claim implies that $l u_{1}^{-1} \stackrel{\circ}{X}_{u_{1}}^{G / P} \cap u_{2}^{-1} \stackrel{\circ}{X}_{u_{2}}^{G / P} \cap v^{-1} \stackrel{\circ}{X}_{v}^{G / P}$ is finite. Then Proposition 2 allows to conclude. 
9.3. About $\Gamma(\mathfrak{g})$. For any $n \in \mathrm{z}, L(n \delta)$ is one dimensional acted on by the character $n \delta$ of $\mathfrak{g}$. It follows that

$$
\Gamma(\mathfrak{g})=\Gamma_{\text {red }}(\mathfrak{g})+\mathrm{Q}(\delta, 0, \delta)+\mathrm{Q}(0, \delta, \delta),
$$

where

$$
\Gamma_{\text {red }}(\mathfrak{g})=\left\{\left(\lambda_{1}, \lambda_{2}, \mu\right) \in \Gamma(\mathfrak{g}): \lambda_{1}(d)=\lambda_{2}(d)=0\right\} .
$$

For any $\lambda \in P_{+}$, the center $\mathrm{C} c$ of $\mathfrak{g}$ acts on $L(\lambda)$ with weight $\lambda(c) \in \mathrm{z}$. Then

$$
\Gamma_{\text {red }}(\mathfrak{g}) \subset \Gamma(\mathfrak{g}) \subset\left\{\left(\lambda_{1}, \lambda_{2}, \mu\right) \in\left(\mathfrak{h}_{Q}^{*}\right)^{3}: \mu(c)=\lambda_{1}(c)+\lambda_{2}(c)\right\} .
$$

As an application of the GKO construction [GKO85] of representations of Virasoro algebras, Kac-Wakimoto obtained in KW88 the following properties of decomposition of $L\left(\lambda_{1}\right) \otimes L\left(\lambda_{2}\right)$.

Lemma 26. Let $\lambda_{1}, \lambda_{2}$ in $P_{+}$such that $\lambda_{1}(d)=\lambda_{2}(d)=0, \lambda_{1}(c)>0$ and $\lambda_{2}(c)>0$. Let $\dot{\mu} \in \dot{P}$ and set $\bar{\mu}:=\dot{\mu}+\left(\lambda_{1}(c)+\lambda_{2}(c)\right) \Lambda \in P_{+}$.

Then, there exists $b \in \mathrm{Z}$ such that $L(\bar{\mu}+b \delta)$ is a submodule of $L\left(\lambda_{1}\right) \otimes L\left(\lambda_{2}\right)$ if and only if $\bar{\mu}-\lambda_{1}-\lambda_{2} \in Q$.

Moreover, if $\bar{\mu}-\lambda_{1}-\lambda_{2} \in Q$ then one of the following two assertions holds:

(i) there exists $b_{0} \in \mathrm{Z}$ such that $L(\bar{\mu}+b \delta) \subset L\left(\lambda_{1}\right) \otimes L\left(\lambda_{2}\right)$ if and only if $b \leq b_{0}$;

(ii) there exists $b_{0} \in \mathrm{Z}$ such that $L(\bar{\mu}+b \delta) \subset L\left(\lambda_{1}\right) \otimes L\left(\lambda_{2}\right)$ if and only if $b=b_{0}$ or $b \in b_{0}-\mathrm{z}_{>1}$.

Set

$$
b_{0}\left(\lambda_{1}, \lambda_{2}, \bar{\mu}\right)=\max \left\{b \in \mathrm{Z}: L(\bar{\mu}+b \delta) \subset L\left(\lambda_{1}\right) \otimes L\left(\lambda_{2}\right)\right\} .
$$

Proof. The first assertion is proved in [KW88, p. 194]. The fact that $\left\{b \in \mathrm{Z}: L(\bar{\mu}+b \delta) \subset L\left(\lambda_{1}\right) \otimes L\left(\lambda_{2}\right)\right\}$ has an upper bound is proved on [KW88, p. 171]. Let $b_{0}$ be the maximum of such $b \in \mathrm{Z}$. It remains to prove that $b_{0}-n \delta$ for all $n \geq 2$. This is a direct consequence of [KW88, Proof of Proposition 3.2]. See also [BK14, Proposition 4.2].

\section{Remark.}

(i) [KW88, Inequality 2.4.1] implies that

$$
b_{0} \leq \frac{\left(\dot{\lambda}_{1}+2 \dot{\rho}, \dot{\lambda}_{1}\right)}{2\left(\ell_{1}+h^{\vee}\right)}+\frac{\left(\dot{\lambda}_{2}+2 \dot{\rho}, \dot{\lambda}_{2}\right)}{2\left(\ell_{2}+h^{\vee}\right)}-\frac{(\dot{\mu}+2 \dot{\rho}, \dot{\mu})}{2\left(\ell_{1}+\ell_{2}+h^{\vee}\right)} .
$$

This inequality is quadratic is $\left(\lambda_{1}, \lambda_{2}, \bar{\mu}\right)$. In this paper, we show stronger linear inequalities.

(ii) If one takes $\ell_{1}=0$ in Lemma 26, one get $\dot{\lambda}_{1}=0$. Set $\lambda_{1}=\dot{\lambda}_{1}+\ell_{1} \Lambda=0, \lambda_{2}=\dot{\lambda}_{2}+\ell_{2} \Lambda$ and $\mu=\dot{\mu}+\ell_{2} \Lambda$. We have $L(N 0) \otimes L\left(N \lambda_{2}\right)=L\left(N \lambda_{2}\right)$, for any positive integer $N$. 
Hence $\left(0, \lambda_{2}, \mu\right)$ belongs to $\Gamma(\mathfrak{g})$ if and only if $\mu=\lambda_{2}$. In particular, the assumption " $\ell_{1}$ positive" is necessary in Lemma 26. Observe that this implies that $\Gamma(\mathfrak{g})$ is not closed.

Set

$$
\Gamma_{\text {red }}^{\circ}(\mathfrak{g})=\left\{\left(\lambda_{1}, \lambda_{2}, \mu\right) \in \Gamma_{\text {red }}(\mathfrak{g}): \lambda_{1}(c)>0 \quad \text { and } \quad \lambda_{2}(c)>0\right\},
$$

and

$$
\begin{aligned}
& \mathcal{A}=\left\{\left(\lambda_{1}, \lambda_{2}, \bar{\mu}\right) \in\left(X(\dot{T})_{Q} \oplus Q \Lambda\right)^{3}: \quad \lambda_{1}, \lambda_{2} \text { and } \bar{\mu}\right. \text { are dominant } \\
& \lambda_{1}(c)>0, \lambda_{2}(c)>0 \\
& \bar{\mu}(c)=\lambda_{1}(c)+\lambda_{2}(c) \\
& \} \text {. }
\end{aligned}
$$

Define a function $\Psi: \mathcal{A} \longrightarrow \mathrm{R}$ by

$$
\Psi\left(\lambda_{1}, \lambda_{2}, \bar{\mu}\right)=\sup _{\substack{N \in \mathrm{Z}_{>0} \text { s.t. } \\ N \lambda_{1}, N \lambda_{2}, N \bar{\mu} \in \mathfrak{h}_{\mathrm{z}}^{*}}} \frac{b_{0}\left(N \lambda_{1}, N \lambda_{2}, N \bar{\mu}\right)}{N},
$$

where $b_{0}$ is defined in Lemma 26. This lemma implies that $\left(\lambda_{1}, \lambda_{2}, \mu+\right.$ $b \delta$ ) belongs to the closure of $\Gamma_{\text {red }}^{\circ}(\mathfrak{g})$ in $\mathcal{A} \times \mathrm{R}$ if and only if

$$
b \leq \Psi\left(\lambda_{1}, \lambda_{2}, \bar{\mu}\right) .
$$

9.4. A cone defined by inequalities. Consider the cone $\mathcal{C}$ of points $\left(\lambda_{1}, \lambda_{2}, \mu\right) \in\left(\mathfrak{h}_{Q}^{*}\right)^{3}$ such that

(i) $\lambda_{1}(c)>0$ and $\lambda_{2}(c)>0$;

(ii) $\lambda_{1}, \lambda_{2}$, and $\mu$ are dominant;

(iii) $\lambda_{1}(d)=\lambda_{2}(d)=0$;

(iv) $\lambda_{1}(c)+\lambda_{2}(c)=\mu(c)$;

(v) the inequality

$$
\left\langle\mu, v \varpi_{\alpha_{i}^{\vee}}\right\rangle \leq\left\langle\lambda_{1}, u_{1} \varpi_{\alpha_{i}^{\vee}}\right\rangle+\left\langle\lambda_{2}, u_{2} \varpi_{\alpha_{i}^{\vee}}\right\rangle
$$

holds for any $i \in\{0, \ldots, l\}$ and any $\left(u_{1}, u_{2}, v\right) \in W^{P_{i}}$ such that

The aim of this section is to prove Theorem 1 or equivalently Theorem 7 below. We first study the cone $\mathcal{C}$.

9.5. Realisation of $\mathcal{C}$ as an hypograph. For $\mu \in X(\dot{T})_{Q} \oplus Q \Lambda \oplus \mathrm{Q} \delta$, we denote by $\dot{\mu}($ resp. $\bar{\mu})$ its projection on $X(\dot{T})_{Q}\left(\operatorname{resp} . X(\dot{T})_{Q} \oplus \mathrm{Q} \Lambda\right)$.

Let $\mathcal{I}$ be the set of $\left(u_{1}, u_{2}, v, i\right) \in\left(W^{P_{i}}\right)^{3} \times\{0, \ldots l\}$ satisfying condition (23). Fix $\left(u_{1}, u_{2}, v, i\right) \in \mathcal{I}$. Assume first that $i=0$. Let $h_{1}, h_{2}$ and $h$ in $\dot{Q}^{\vee}$ such that $u_{1} W_{P_{0}}=h_{1} W_{P_{0}}, u_{2} W_{P_{0}}=h_{2} W_{P_{0}}$ and $v W_{P_{0}}=h W_{P_{0}}$. 
Define the (restriction of) linear function $\varphi_{\left(u_{1}, u_{2}, v, 0\right)}: \mathcal{A} \longrightarrow \mathrm{Q}$ that maps $\left(\lambda_{1}, \lambda_{2}, \bar{\mu}\right)$ to

$$
\begin{aligned}
\left\langle h_{1}, \dot{\lambda}_{1}\right\rangle+\left\langle h_{2}, \dot{\lambda}_{2}\right\rangle-\langle h, \dot{\mu}\rangle & \\
& +\frac{\ell_{1}}{2}\left(\|h\|^{2}-\left\|h_{1}\right\|^{2}\right)+\frac{\ell_{2}}{2}\left(\|h\|^{2}-\left\|h_{2}\right\|^{2}\right),
\end{aligned}
$$

where $\ell_{1}=\lambda_{1}(c)$ and $\ell_{2}=\lambda_{2}(c)$. Note that $\varpi_{\alpha_{0}^{\vee}}=d$ and for $h \in \dot{Q}^{\vee}$, by equation (19), we have $h \cdot d=h+d-\frac{(h, h)}{2} c$. Let $\left(\lambda_{1}, \lambda_{2}, \bar{\mu}\right) \in \mathcal{A}$. Then inequality (22) with $i=0$, is fulfilled by $\left(\lambda_{1}, \lambda_{2}, \bar{\mu}+b \delta\right)$ if and only if

$$
b \leq \varphi_{\left(u_{1}, u_{2}, v, 0\right)}\left(\lambda_{1}, \lambda_{2}, \bar{\mu}\right) .
$$

Assume now that $i \in\{1, \ldots, l\}$. Write $u_{1}=\dot{u}_{1} h_{1}, u_{2}=\dot{u}_{2} h_{2}$ and $v=\dot{v} h$ with $\dot{u}_{1}, \dot{u}_{2}, \dot{v} \in \dot{W}$ and $h_{1}, h_{2}, h \in \dot{Q}^{\vee}$. Define the linear function $\varphi_{\left(u_{1}, u_{2}, v, i\right)}: \mathcal{A} \longrightarrow \mathrm{Q}$ that maps $\left(\lambda_{1}, \lambda_{2}, \bar{\mu}\right)$ to

$$
\begin{aligned}
& \left\langle\dot{u}_{1}\left(h_{1}+\frac{\varpi_{\alpha_{i}^{\vee}}}{\left\langle\dot{\varpi}_{\alpha_{i}^{\vee}}, \dot{\theta}\right\rangle}\right), \dot{\lambda}_{1}\right\rangle+\left\langle\dot{u}_{2}\left(h_{2}+\frac{\varpi_{\alpha_{i}^{\vee}}}{\left\langle\dot{\varpi}_{\alpha_{i}^{\vee}}, \dot{\theta}\right\rangle}\right), \dot{\lambda}_{2}\right\rangle-\left\langle\dot{v}\left(h+\frac{\varpi_{\alpha_{i}^{\vee}}}{\left\langle\dot{\varpi}_{\alpha_{i}^{\vee}}, \dot{\theta}\right\rangle}\right), \dot{\mu}\right\rangle \\
& +\frac{\ell_{1}}{2}\left(\|h\|^{2}-\left\|h_{1}\right\|^{2}+2 \frac{\left(\varpi_{\alpha_{i}^{\vee}}, h-h_{1}\right)}{\left\langle\dot{\varpi}_{v_{i}}, \dot{\theta}\right\rangle}\right) \\
& +\frac{\ell_{2}}{2}\left(\|h\|^{2}-\left\|h_{2}\right\|^{2}+2 \frac{\left(\varpi_{\left.\alpha_{i}^{\vee}, h-h_{2}\right)}\right.}{\left\langle\dot{\varpi}_{\alpha_{i}^{\vee}}, \dot{\theta}\right\rangle}\right) .
\end{aligned}
$$

Recall that $\varpi_{\alpha_{i}^{\vee}}=\dot{\varpi}_{\alpha_{i}^{\vee}}+\left\langle\dot{\varpi}_{\alpha_{i}^{\vee}}, \dot{\theta}\right\rangle d$. Moreover, for $w=\dot{w} h \in W$, by equation (19), we have

$(\dot{w} h) \cdot \varpi_{\alpha_{i}^{\vee}}=\dot{w} \dot{\varpi}_{\alpha_{i}^{\vee}}+\left\langle\dot{\varpi}_{\alpha_{i}^{\vee}}, \dot{\theta}\right\rangle \dot{w} h+\left\langle\dot{\varpi}_{\alpha_{i}^{\vee}}, \dot{\theta}\right\rangle d-\left(\left\langle\dot{\varpi}_{\alpha_{i}^{\vee}}, \dot{\theta}\right\rangle \frac{(h, h)}{2}+\left(\dot{\varpi}_{\alpha_{i}^{\vee}}, h\right)\right) c$.

Then inequality (22) , is fulfilled by $\left(\lambda_{1}, \lambda_{2}, \bar{\mu}+b \delta\right)$ if and only if

$$
b \leq \varphi_{\left(u_{1}, u_{2}, v, i\right)}\left(\lambda_{1}, \lambda_{2}, \bar{\mu}\right) \text {. }
$$

Define

$$
\begin{aligned}
& \varphi: \mathcal{A} \quad \longrightarrow \mathrm{R} \cup\{-\infty\} \\
& \left(\lambda_{1}, \lambda_{2}, \bar{\mu}\right) \longmapsto \inf _{\left(u_{1}, u_{2}, v, i\right) \in \mathcal{I}} \varphi_{\left(u_{1}, u_{2}, v, i\right)}\left(\lambda_{1}, \lambda_{2}, \bar{\mu}\right) .
\end{aligned}
$$

Then $\varphi$ is a concave function and $\mathcal{C}$ is the hypograph of $\varphi$ :

$$
\mathcal{C}=\left\{\left(\lambda_{1}, \lambda_{2}, \bar{\mu}+b \delta\right):\left(\lambda_{1}, \lambda_{2}, \bar{\mu}\right) \in \mathcal{A} \text { and } b \leq \varphi\left(\lambda_{1}, \lambda_{2}, \bar{\mu}\right)\right\} .
$$

\subsection{The convex set $\mathcal{C}$ is locally polyhedral.}

Proposition 4. Let $x_{0} \in \mathcal{A}$. Then

$$
\forall M \in \mathrm{R} \quad \exists \text { an open } U \ni x_{0} \quad \exists J \subset \mathcal{I} \text { finite }
$$

such that

$$
\forall x \in U \quad \forall a \in \mathcal{I} \quad \varphi_{a}(x)<M \Rightarrow a \in J .
$$


Proof. Fix $M \in$ R. Let $\left(u_{1}, u_{2}, v, 0\right) \in \mathcal{I}$. By Proposition 1, the inequality (22) is satisfied by $(\ell \Lambda, 0, \ell \Lambda)$ for any $\ell>0$. Hence

$$
\|h\|^{2}-\left\|h_{1}\right\|^{2} \geq 0 \text {. }
$$

Similarly, $\|h\|^{2}-\left\|h_{2}\right\|^{2} \geq 0$. Hence, the image of $\left(\lambda_{1}, \lambda_{2}, \bar{\mu}\right) \in \mathcal{A}$ by $\varphi_{\left(u_{1}, u_{2}, v, 0\right)}$ is greater or equal to

$$
\left\langle h_{1}, \dot{\lambda}_{1}\right\rangle+\left\langle h_{2}, \dot{\lambda}_{2}\right\rangle-\langle h, \dot{\mu}\rangle+\frac{l}{2}\left(2\|h\|^{2}-\left\|h_{1}\right\|^{2}-\left\|h_{2}\right\|^{2}\right)
$$

where $\ell=\min \left(\lambda_{1}(c), \lambda_{2}(c)\right)$. Using Lemma 18, one gets $\delta_{u_{1} u_{2}}^{v}=$ $\left\langle\rho,-d-h \cdot d+h_{1} \cdot d+h_{2} \cdot d\right\rangle$. Hence

$$
\delta_{u_{1} u_{2}}^{v}=\left\langle\dot{\rho}, h_{1}+h_{2}-h\right\rangle+\frac{h^{\vee}}{2}\left(\|h\|^{2}-\left\|h_{1}\right\|^{2}-\left\|h_{2}\right\|^{2}\right) .
$$

Lemma 17 implies that $\delta_{u_{1} u_{2}}^{v} \geq 0$ and

$$
\|h\|^{2}-\left\|h_{1}\right\|^{2}-\left\|h_{2}\right\|^{2} \geq \frac{2}{h^{v}}\left\langle h-h_{1}-h_{2}, \dot{\rho}\right\rangle .
$$

Then the image of $\left(\lambda_{1}, \lambda_{2}, \bar{\mu}\right) \in \mathcal{A}$ by $\varphi_{\left(u_{1}, u_{2}, v, 0\right)}$ is greater or equal to

$\frac{\ell}{2}\|h\|^{2}-\left\|h_{1}\right\| \cdot\left\|\dot{\lambda}_{1}\right\|-\left\|h_{2}\right\| \cdot\left\|\dot{\lambda}_{2}\right\|-\|h\| \cdot\|\dot{\mu}\|-\frac{2\|\dot{\rho}\|}{h^{\vee}}\left(\left\|h_{1}\right\|+\left\|h_{2}\right\|+\|h\|\right)$.

By construction there exist $\dot{u}_{1}, \dot{u}_{2}$ and $\dot{v}$ in $\dot{W}$ such that $u_{1}=h_{1} \dot{u}_{1}$, $u_{2}=h_{2} \dot{u}_{2}$ and $v=h \dot{v}$. But $l(v)=l\left(u_{1}\right)+l\left(u_{2}\right)$. Then Lemma 11.4 implies that

$$
N+\sqrt{2} N\|h\| \geq l(v) \geq l\left(u_{1}\right) \geq K\left\|h_{1}\right\|-N .
$$

Then

$$
\max \left(\left\|h_{1}\right\|,\left\|h_{2}\right\|\right) \leq \frac{N}{K}(2+\sqrt{2}\|h\|) .
$$

The point is that this implies that $\varphi_{\left(u_{1}, u_{2}, v, 0\right)}\left(\lambda_{1}, \lambda_{2}, \bar{\mu}\right)$ is greater or equal to $\frac{\ell}{2}\|h\|^{2}$ minus terms that are linear in $\|h\|$. One can easily deduce that there exist an open neighborhood $U_{0}$ of $x_{0}$ and $A_{0} \in \mathrm{R}$ such that

$$
\forall x \in U_{0} \quad \forall a=\left(u_{1}, u_{2}, v, 0\right) \in \mathcal{I} \quad l(v) \geq A_{0} \Rightarrow \varphi_{a}(x) \geq M .
$$

Let $\left(u_{1}, u_{2}, v, i\right) \in \mathcal{I}$ with $i>0$ and consider the associated linear function $\varphi_{\left(u_{1}, u_{2}, v, i\right)}$. Since $(\ell \Lambda, 0, \ell \Lambda) \in \Gamma(\mathfrak{g})$ for any $\ell>0$, Proposition 1 implies that

$$
\|h\|^{2}-\left\|h_{1}\right\|^{2}+2 \frac{\left(\varpi_{\alpha_{i}^{\vee}}, h-h_{1}\right)}{\left\langle\dot{\varpi}_{\alpha_{i}^{\vee}}, \dot{\theta}\right\rangle} \geq 0 .
$$


Set $C:=\frac{\left\|\dot{\varpi}_{\alpha_{i}^{\vee}}\right\|}{\left\langle\dot{\varpi}_{\alpha_{i}}, \dot{\theta}\right\rangle}$ and $D=\left\langle\dot{\varpi}_{\alpha_{i}^{\vee}}, \dot{\theta}\right\rangle$. Then, the image of $\left(\lambda_{1}, \lambda_{2}, \bar{\mu}\right) \in \mathcal{A}$ by $\varphi_{\left(u_{1}, u_{2}, v, i\right)}$ is greater or equal to

$$
\begin{aligned}
& -\left(\left\|h_{1}\right\| \cdot\left\|\dot{\lambda}_{1}\right\|+\left\|h_{2}\right\| \cdot\left\|\dot{\lambda}_{2}\right\|+\|h\| \cdot\|\dot{\mu}\|\right)-C\left(\left\|\dot{\lambda}_{1}\right\|+\left\|\dot{\lambda}_{2}\right\|+\|\dot{\mu}\|\right) \\
& +\frac{\ell}{2}\left(2\|h\|^{2}-\left\|h_{1}\right\|^{2}-\left\|h_{2}\right\|^{2}-2 C\left(\left\|h-h_{1}\right\|+\left\|h-h_{2}\right\|\right)\right) .
\end{aligned}
$$

where $\ell=\min \left(\lambda_{1}(c), \lambda_{2}(c)\right)$. But $\delta_{u_{1} u_{2}}^{v} \geq 0$ implies that

$$
\begin{aligned}
\|h\|^{2}-\left\|h_{1}\right\|^{2}-\left\|h_{2}\right\|^{2} \geq & \frac{2}{D h^{\bigvee}}\left(1-\left\langle\dot{\rho},-\dot{v} \dot{\varpi}_{\alpha_{i}^{\vee}}+\dot{u}_{1} \dot{\varpi}_{\alpha_{i}^{\vee}}+\dot{u}_{2} \dot{\varpi}_{\alpha_{i}^{\vee}}\right\rangle\right) \\
& -\frac{2}{h^{\vee}}\left(\left\langle\dot{\rho},-\dot{v} h+\dot{u}_{1} h_{1}+\dot{u}_{2} h_{2}\right\rangle\right) \\
& -\frac{2}{D}\left(\dot{\varpi}_{\alpha_{i}^{\vee}}, h-h_{1}-h_{2}\right) .
\end{aligned}
$$

Combining these inequalities with inequality (28) one can get a lower bound for $\varphi_{\left(u_{1}, u_{2}, v, i\right)}\left(\lambda_{1}, \lambda_{2}, \bar{\mu}\right)$ equals to $\frac{\ell}{2}\|h\|^{2}$ minus terms that are linear in $\|h\|$. One can easily deduce that there exist an open neighborhood $U$ of $x_{0}$ and $A \in \mathrm{R}$ such that

$$
\forall x \in U \quad \forall a=\left(u_{1}, u_{2}, v, i\right) \in \mathcal{I} \quad l(v) \geq A \Rightarrow \varphi_{a}(x) \geq M .
$$

But there exist only finitely many triples $\left(h_{1} \dot{u}_{1}, h_{2} \dot{u}_{2}, h \dot{v}\right)$ with $l(v)<$ $A$ and $l(v)=l\left(u_{1}\right)+l\left(u_{2}\right)$. The proposition follows.

Remark. Proposition 4 is still true with the family of equations corresponding to $P$ any parabolic subgroup, $\alpha_{i} \in \Delta-\Delta(P)$ and $n_{u_{1} u_{2}}^{v} \neq$ 0 . The same proof works.

For any $\left(u_{1}, u_{2}, v, i\right) \in \mathcal{I}$, we set

$$
\mathcal{A}_{\left(u_{1}, u_{2}, v, i\right)}=\left\{\left(\lambda_{1}, \lambda_{2}, \bar{\mu}\right) \in \mathcal{A}: \varphi\left(\lambda_{1}, \lambda_{2}, \bar{\mu}\right)=\varphi_{\left(u_{1}, u_{2}, v, i\right)}\left(\lambda_{1}, \lambda_{2}, \bar{\mu}\right)\right\} .
$$

The properties of these sets are summarized in the following lemma. The dimension of a convex set is the dimension of the generated affine space.

Lemma 27. With above notation,

(i) The sets $\mathcal{A}_{\left(u_{1}, u_{2}, v, i\right)}$ are convex.

(ii) $\operatorname{Set} \mathcal{I}_{1}=\left\{\left(u_{1}, u_{2}, v, i\right) \in \mathcal{I} \mid \operatorname{dim}\left(\mathcal{A}_{\left(u_{1}, u_{2}, v, i\right)}\right)=\operatorname{dim}(\mathcal{A})\right\}$. Then

$$
\mathcal{A}=\cup_{\left(u_{1}, u_{2}, v, i\right) \in \mathcal{I}_{1}} \mathcal{A}_{\left(u_{1}, u_{2}, v, i\right)} .
$$

(iii) The sets associated to two different elements of $\mathcal{I}_{1}$ only intersect along their boundaries.

Proof. The first assertion follows from the linearity of functions $\varphi_{\left(u_{1}, u_{2}, v, i\right)}$. Proposition 4 implies that $\mathcal{C}$ is locally polyhedral and the two last assertions. 
For any $\left(u_{1}, u_{2}, v, i\right) \in \mathcal{I}$, we set

$$
\begin{aligned}
\mathcal{F}_{\left(u_{1}, u_{2}, v, i\right)}=\left\{\left(\lambda_{1}, \lambda_{2}, \bar{\mu}+b \delta\right):\right. & \left(\lambda_{1}, \lambda_{2}, \bar{\mu}\right) \in \mathcal{A}_{\left(u_{1}, u_{2}, v, i\right)} \\
& \left.b=\varphi_{\left(u_{1}, u_{2}, v, i\right)}\left(\lambda_{1}, \lambda_{2}, \bar{\mu}\right)\right\} .
\end{aligned}
$$

Then

$$
\left\{\mathcal{F}_{\left(u_{1}, u_{2}, v, i\right)}:\left(u_{1}, u_{2}, v, i\right) \in \mathcal{I}_{1}\right\}
$$

is the collection of codimension one faces of $\mathcal{C}$. Moreover,

$$
\begin{aligned}
\mathcal{C}=\left\{\left(\lambda_{1}, \lambda_{2}, \bar{\mu}+b \delta\right):\right. & \left(\lambda_{1}, \lambda_{2}, \bar{\mu}\right) \in \mathcal{A} \\
& \left.b \leq \varphi_{\left(u_{1}, u_{2}, v, i\right)}\left(\lambda_{1}, \lambda_{2}, \bar{\mu}\right) \quad \forall\left(u_{1}, u_{2}, v, i\right) \in \mathcal{I}_{1}\right\} .
\end{aligned}
$$

Two convex sets $\mathcal{A}_{\left(u_{1}, u_{2}, v, i\right)}$ and $\mathcal{A}_{\left(u_{1}^{\prime}, u_{2}^{\prime}, v^{\prime}, i\right)}$ are said to be adjacent if their intersection has codimension 1.

9.7. An example of a face of codimension 1. Consider the element $(e, e, e, 0) \in \mathcal{I}$. The associated inequality (22) is $b \leq 0$. Moreover, $G / P_{0}$ is the affine Grassmannian $\mathcal{G} r_{\dot{G}}$, the semi-simple component of the Levi subgroup $L_{0}$ is $\dot{G}$.

Lemma 28. Let $\left(\dot{\lambda}_{1}+\ell_{1} \Lambda, \dot{\lambda}_{2}+\ell_{2} \Lambda, \dot{\mu}+\left(\ell_{1}+\ell_{2}\right) \Lambda\right) \in\left(P_{+}\right)^{3}$. Then $L\left(\dot{\mu}+\left(\ell_{1}+\ell_{2}\right) \Lambda\right)$ is contained in $L\left(\dot{\lambda}_{1}+\ell_{1} \Lambda\right) \otimes L\left(\dot{\lambda}_{2}+\ell_{2} \Lambda\right)$ if and only if $L_{\dot{G}}(\dot{\mu})$ is contained in $L_{\dot{G}}\left(\dot{\lambda}_{1}\right) \otimes L_{\dot{G}}\left(\dot{\lambda}_{2}\right)$.

In particular, $\mathcal{A}_{(e, e, e, 0)}$ has nonempty interior in $\mathcal{A}$.

Proof. The first assertion is certainly well known. It can also be obtained as a consequence of Theorem 5. Indeed, in $\mathrm{H}^{*}\left(\mathcal{G} r_{\dot{G}}, \mathrm{z}\right)$, we have $n_{e, e}^{e}=1$. For $\tau=\varpi_{\alpha_{0}^{\vee}},\left(\dot{\lambda}_{1}+\ell_{1} \Lambda, \dot{\lambda}_{2}+\ell_{2} \Lambda, \dot{\mu}+\left(\ell_{1}+\ell_{2}\right) \Lambda\right)$ satisfies equality (11). Corollary 1 shows that the multiplicity of $L\left(\dot{\mu}+\left(\ell_{1}+\ell_{2}\right) \Lambda\right)$ in $L\left(\dot{\lambda}_{1}+\ell_{1} \Lambda\right) \otimes L\left(\dot{\lambda}_{2}+\ell_{2} \Lambda\right)$ is equal to those of $L_{\dot{G}}(\dot{\mu})$ in $L_{\dot{G}}\left(\dot{\lambda}_{1}\right) \otimes L_{\dot{G}}\left(\dot{\lambda}_{2}\right)$. The first assertion of the lemma follows.

It is well known (see e.g. [PR13, Theorem 1.4]) that $\Gamma(\dot{\mathfrak{g}})$ has nonempty interior in $\left(X(\dot{T})_{Q}\right)^{3}$. But for any given $\left(\dot{\lambda}_{1}, \dot{\lambda}_{2}, \dot{\mu}\right) \in \Gamma(\dot{\mathfrak{g}})$, $\left(\dot{\lambda}_{1}+\ell_{1} \Lambda, \dot{\lambda}_{2}+\ell_{2} \Lambda, \dot{\mu}+\left(\ell_{1}+\ell_{2}\right) \Lambda\right) \in \Gamma(\mathfrak{g})$ for any $\ell_{1} \geq\left\langle\dot{\lambda}_{1}, \dot{\theta}^{\vee}\right\rangle$, $\ell_{2} \geq\left\langle\dot{\lambda}_{2}, \dot{\theta}^{\vee}\right\rangle$ and $\ell_{1}+\ell_{2} \geq\left\langle\dot{\mu}, \dot{\theta}^{\vee}\right\rangle$. The second assertion follows.

9.8. The main result. We can now restate our description $\Gamma^{\circ}(\mathfrak{g})$.

Theorem 7. With the above notation, we have

$$
\Gamma_{\text {red }}^{\circ}(\mathfrak{g})=\mathcal{C} .
$$

Proof. The inclusion $\Gamma_{\text {red }}^{\circ}(\mathfrak{g}) \subset \mathcal{C}$ is a direct consequence of Proposition 1. We have to prove that $\mathcal{C}$ is contained in $\Gamma(\mathfrak{g})$. Let $x_{0} \in \mathcal{A}$. By Lemma [26, to show that $\left.\left.x_{0}+\right]-\infty, \varphi(x)\right](0,0, \delta)$ is contained in $\Gamma(\mathfrak{g})$, it is sufficient to prove that $x_{0}+\varphi(x)(0,0, \delta)$ belongs to $\Gamma(\mathfrak{g})$.

By Proposition 4, there exists $a_{0} \in \mathcal{I}$ such that $\varphi\left(x_{0}\right)=\varphi_{a_{0}}\left(x_{0}\right)$. Let $U$ be an open neighborhood of $x_{0}$ in $\mathcal{A}$ such that for any $y \in U, \varphi_{a_{0}}(y) \leq$ 
$\varphi\left(x_{0}\right)+1$. By Proposition 4, there exists a smaller neighborhood $V \subset U$ of $x_{0}$ and $J \subset \mathcal{I}$ finite such that

$$
\forall y \in V \quad \forall a \in \mathcal{I}-J \quad \varphi_{a}(y)>\varphi\left(x_{0}\right)+1 \geq \varphi_{a_{0}}(y) .
$$

Note that $a_{0} \in J$. Then, for any $y \in V$

$$
\varphi(y)=\min _{a \in J} \varphi_{a}(y) .
$$

Choose a simplex $\mathcal{S}$ containing $x_{0}$ in its interior such that $\mathcal{S} \cap \mathcal{A} \subset V$. Up to replacing $V$ by $\mathcal{S} \cap \mathcal{A}$, one may assume that $V$ is a convex polytope. Then, the theory of polyhedrons implies that, for any $y \in V$

$$
\varphi(y)=\min _{a \in J \cap \mathcal{I}_{1}} \varphi_{a}(y) .
$$

In particular, it is sufficient to prove that

$$
\forall\left(u_{1}, u_{2}, v, i\right) \in \mathcal{I}_{1} \quad \mathcal{F}_{\left(u_{1}, u_{2}, v, i\right)} \subset \Gamma_{\text {red }}^{\circ}(\mathfrak{g}) .
$$

Let $\mathcal{I}_{1}^{0}$ denote the set of elements in $\mathcal{I}_{1}$ satisfying inclusion (32).

Fix an element $\left(u_{1}, u_{2}, v, i\right) \in \mathcal{I}$. Set $C=L_{i} u_{1}^{-1} \underline{\underline{O}}^{-} \times L_{i} u_{2}^{-1} \underline{O}^{-} \times L_{i} v^{-1} \underline{Q}$ the component of $\varpi_{\alpha_{i}^{\vee}}$-fixed points in X. Let $\Gamma\left(C, L_{i}\right)$ denote the set of $\left(\lambda_{1}, \lambda_{2}, \bar{\mu}+b \delta\right) \in \mathcal{A} \times \mathcal{Q}(0,0, \delta)$ such that $C^{\mathrm{ss}}\left(\mathcal{L}, L_{i}\right) \neq \emptyset$ (where $\mathcal{L}$ is the $G$-linearized line bundle on $\mathrm{X}$ associated to $\left.\left(\lambda_{1}, \lambda_{2}, \bar{\mu}+b \delta\right)\right)$. Observe that, since $\varpi_{\alpha_{i}^{\vee}}$ acts trivially on $\mathcal{L}_{\mid C}$, we have $b=\varphi_{\left(u_{1}, u_{2}, v, i\right)}\left(\lambda_{1}, \lambda_{2}, \bar{\mu}\right)$ if $C^{\mathrm{ss}}\left(\mathcal{L}, L_{i}\right) \neq \emptyset$.

Consider the following assumption

$$
\mathcal{F}_{\left(u_{1}, u_{2}, v, i\right)} \cap\left(P_{Q}^{++}\right)^{3} \cap \Gamma(\mathfrak{g}) \text { is not empty. }
$$

The proof proceed in three steps:

Claim 1. Under assumption $(H)$, we have $\left(u_{1}, u_{2}, v, i\right) \in \mathcal{I}_{1}^{0}$.

Claim 2. The element $(e, e, e, 0)$ belongs to $\mathcal{I}_{1}$ and satisfies assumption $(H)$.

Claim 3. If one of two adjacent elements of $\mathcal{I}_{1}$ satisfies assumption $(H)$ then the two ones satisfy assumption $(H)$.

These claims are sufficient. Indeed, for any $a \in \mathcal{I}_{1}$, there exists a sequence $a=a_{0}, \ldots, a_{n}=(e, e, e, 0)$ such that $\mathcal{A}_{a_{i}}$ and $\mathcal{A}_{a_{i+1}}$ are adjacent for any $i$. By Claim 2, $a_{n}$ satisfies assumption $(H)$. By an immediate induction, Claim 3 implies that $a$ satisfies $(H)$. Now Claim 1 shows that $a \in \mathcal{I}_{1}^{0}$.

Proof of Claim 2. It is a direct consequence of Lemma 28.

Proof of Claim $1 \Rightarrow$ Claim 3. Let $a$ and $a^{\prime}$ in $\mathcal{I}_{1}$ be such that $\mathcal{A}_{a}$ and $\mathcal{A}_{a^{\prime}}$ are adjacent along the face $\mathcal{A}^{\prime}$. Assume that a satisfy assumption $(H)$. Then the interior of $\mathcal{A}^{\prime}$ is contained in the interior of $\mathcal{A}_{a} \cup \mathcal{A}_{a^{\prime}}$. In particular, $\mathcal{A}^{\prime}$ intersects $\left(P_{Q}^{++}\right)^{3}$. Let $\left(\lambda_{1}, \lambda_{2}, \bar{\mu}\right) \in \mathcal{A} \cap P^{++}$. Then $\varphi_{a}\left(\lambda_{1}, \lambda_{2}, \bar{\mu}\right)=\varphi_{a^{\prime}}\left(\lambda_{1}, \lambda_{2}, \bar{\mu}\right)$. But, by Claim 1 for $a,\left(\lambda_{1}, \lambda_{2}, \bar{\mu}\right)+$ 
$\varphi_{a}\left(\lambda_{1}, \lambda_{2}, \bar{\mu}\right) \delta$ belongs to $\Gamma(\mathfrak{g})$. Then $\left(\lambda_{1}, \lambda_{2}, \bar{\mu}\right)+\varphi_{a^{\prime}}\left(\lambda_{1}, \lambda_{2}, \bar{\mu}\right) \delta$ belongs to $\Gamma(\mathfrak{g}) \cap P_{Q}^{++} \cap \mathcal{F}_{a^{\prime}}$ and $a^{\prime}$ satisfies assumption $(H)$.

Proof of Claim 1. Let $\left(\bar{u}_{1}, \bar{u}_{2}, \bar{v}, i\right) \in \mathcal{I}_{1}$ satisfying assumption $(H)$. Let $\left(\lambda_{1}, \lambda_{2}, \mu\right) \in \mathcal{F}_{\left(\bar{u}_{1}, \bar{u}_{2}, \bar{v}, i\right)}$. Then $\left(\lambda_{1}, \lambda_{2}, \bar{\mu}\right) \in \mathcal{A}$ and

$$
\mu(d)=\varphi_{\left(\bar{u}_{1}, \bar{u}_{2}, \bar{v}, i\right)}\left(\lambda_{1}, \lambda_{2}, \bar{\mu}\right) .
$$

By Theorem 5, to prove that $\left(\lambda_{1}, \lambda_{2}, \mu\right)$ belongs to $\Gamma(\mathfrak{g})$, it is sufficient to prove that $C^{\mathrm{ss}}\left(\mathcal{L}, L_{i}\right) \neq \emptyset$ where $\mathcal{L}$ is the line bundle on $\mathrm{X}$ associated to $\left(\lambda_{1}, \lambda_{2}, \mu\right)$ and $C=L_{i} u_{1}^{-1} \underline{\underline{O}}^{-} \times L_{i} u_{2}^{-1} \underline{\underline{O}}^{-} \times L_{i} v^{-1} \underline{\underline{O}}$. By [BK06], this last assertion is equivalent to the fact that $\left(\lambda_{1}, \lambda_{2}, \mu\right)$ satisfies a finite family of linear inequalities. More precisely, $C^{\mathrm{ss}}\left(\mathcal{L}, L_{i}\right) \neq \emptyset$ if and only if

(i) $u_{1}^{-1} \lambda_{1}, u_{2}^{-1} \lambda_{2}$ and $v^{-1} \mu$ are dominant for $L_{i}$;

(ii) $Z\left(L_{i}\right)^{\circ}$ acts trivially on $\mathcal{L}_{\mid C}$;

(iii) for any $j \in\{0, \ldots, l\}-\{i\}$

$$
\left\langle\tilde{v} \varpi_{\alpha_{j}^{\vee}}, \bar{v}^{-1} \mu\right\rangle \leq\left\langle\tilde{u}_{1} \varpi_{\alpha_{j}^{\vee}}, \bar{u}_{1}^{-1} \mu\right\rangle+\left\langle\tilde{u}_{2} \varpi_{\alpha_{j}^{\vee}}, \bar{u}_{2}^{-1} \mu\right\rangle,
$$

for any $\left(\tilde{u}_{1}, \tilde{u}_{2}, \tilde{v}\right) \in W_{L_{i}}^{P_{j}^{i}}$ such that $\epsilon_{\tilde{v}}\left(L_{i} / P_{j}^{i}\right)$ appears with coefficient one in $\epsilon_{\tilde{u}_{1}}\left(L_{i} / P_{j}^{i}\right) \odot_{0} \epsilon_{\tilde{u}_{2}}\left(L_{i} / P_{j}^{i}\right)$.

Here $P_{j}^{i}$ is the maximal standard parabolic subgroup of $L_{i}$ associated to $j$. Then $P_{j}^{i}=L_{i} \cap P_{j}$. Set also $P_{i, j}=P_{i} \cap P_{j}$. One easily checks that $\left(\lambda_{1}, \lambda_{2}, \mu\right)$ satisfies (ii) and (iii). It remains to prove that it satisfies inequalities (34).

Denote by $p$ the projection that maps $\left(\lambda_{1}, \lambda_{2}, \mu\right)$ on $\left(\lambda_{1}, \lambda_{2}, \bar{\mu}\right)$. By BK06, the image by $p$ of the set of triples satisfying conditions (ii), (iii) and inequalities (34) is a closed convex polyhedral cone $\mathcal{C}^{L_{i}}(C)$ of nonempty interior. It remains to prove that $\left(\lambda_{1}, \lambda_{2}, \bar{\mu}\right)$ belongs to $\mathcal{C}^{L_{i}}(C) \cap \mathcal{A}$. By assumption $(H)$, the cone $\mathcal{C}^{L_{i}}(C)$ intersects the interior of $\mathcal{A}$. In particular, $\mathcal{C}^{L_{i}}(C) \cap \mathcal{A}$ has nonempty interior. Then, the general theory of convex polyhedrons implies that it is sufficient to check the conditions (34) such that the associated face of $\mathcal{C}^{L_{i}}(C) \cap \mathcal{A}$ has codimension one. Consider such an inequality associated to $\left(\tilde{u}_{1}, \tilde{u}_{2}, \tilde{v}, j\right)$ and

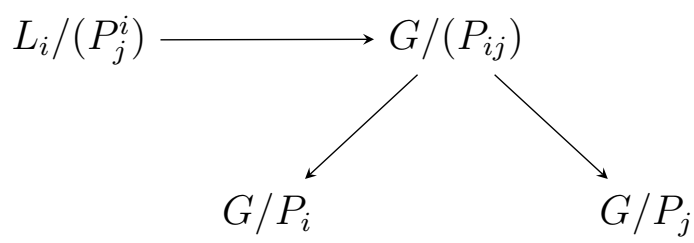

By Lemma 20, $\left(u_{1}=\bar{u}_{1} \tilde{u}_{1}, u_{2}=\bar{u}_{2} \tilde{u}_{2}, v=\bar{v} \tilde{v}\right) \in\left(W^{P_{i, j}}\right)^{3}$. By Proposition 3, the $\epsilon_{v}$ appears with multiplicity one in $\epsilon_{u_{1}} \cdot \epsilon_{u_{2}}$, in $\mathrm{H}^{*}\left(G / P_{i, j}, \mathrm{z}\right)$. 
Set $\tau=\varpi_{\alpha_{i}^{\vee}}+\varpi_{\alpha_{j}^{\vee}}$. Then

$$
\left\langle v \tau, \mu^{\prime}\right\rangle \leq\left\langle u_{1} \tau, \lambda_{1}^{\prime}\right\rangle+\left\langle u_{2} \tau, \lambda_{2}^{\prime}\right\rangle
$$

for any $\left(\lambda_{1}^{\prime}, \lambda_{2}^{\prime}, \mu^{\prime}\right) \in \Gamma(\mathfrak{g})$. Let $\left(\lambda_{1}^{\prime \prime}, \lambda_{2}^{\prime \prime}, \mu^{\prime \prime}\right)$ in the interior of $\mathcal{A}$, in $\mathcal{C}^{L_{i}}(C)$ and such that inequality (34) is an equality. Then inequality (35) is an equality for $\left(\lambda_{1}^{\prime \prime}, \lambda_{2}^{\prime \prime}, \mu^{\prime \prime}+\varphi_{\left(\bar{u}_{1}, \bar{u}_{2}, \bar{v}, i\right)} \delta\right)$. Now, Theorem 6 implies that $\epsilon_{v}$ appears with multiplicity one in $\epsilon_{u_{1}} \odot_{0} \epsilon_{u_{2}}$.

Consider now the three elements $\bar{u}_{1}^{\prime}, \bar{u}_{2}^{\prime}$ and $\bar{v}^{\prime}$ in $W^{P_{j}}$ such that $\bar{u}_{1}^{\prime} W_{P_{j}}=u_{1} W_{P_{j}}, \bar{u}_{2}^{\prime} W_{P_{j}}=u_{2} W_{P_{j}}$ and $\bar{v}^{\prime} W_{P_{j}}=v W_{P_{j}}$. Lemma 23 and Proposition 3 imply that, in $H^{*}\left(G / P_{j}, \mathrm{z}\right), \epsilon_{\bar{v}^{\prime}}$ appears with multiplicity one in $\epsilon_{\bar{u}_{1}^{\prime}} \odot_{0} \epsilon_{\bar{u}_{2}^{\prime}}$. Since $\left(\lambda_{1}, \lambda_{2}, \mu\right) \in \mathcal{C}$, it satisfies

$$
\left\langle\mu, v \varpi_{\alpha_{j}^{\vee}}\right\rangle \leq\left\langle\lambda_{1}, u_{1} \varpi_{\alpha_{j}^{\vee}}\right\rangle+\left\langle\lambda_{2}, u_{2} \varpi_{\alpha_{j}^{\vee}}\right\rangle
$$

But, modulo equality (33), the inequality (34) to prove is equivalent to inequality (36).

We conclude that $\left(\bar{u}_{1}, \bar{u}_{2}, \bar{v}, i\right)$ belongs to $\mathcal{I}_{1}^{0}$.

\section{SATURATION FACTORS}

In this section, we prove Theorems 3 and 4 of the introduction. Let us first check the computation of the constants $k_{s}$.

In the finite dimensional case, known saturation factors are collected in the following tabular. These results was obtained in [KT99] for the type $A$, in [KM06] for $B_{2}$ and $G_{2}$, [BK10] for types $B_{\ell}, C_{\ell}$, [KKM09] for $D 4$ and in [KM08] in the remaning cases.

\begin{tabular}{|c|c|c|c|c|c|}
\hline Type & $A_{\ell}$ & $B_{\ell}(\ell \geq 2)$ & $C_{\ell}(\ell \geq 3)$ & $D_{4}$ & $D_{\ell}(\ell \geq 5)$ \\
\hline Saturation factor & 1 & 2 & 2 & 1 & 4 \\
\hline \hline Type & $E_{6}$ & $E_{7}$ & $E_{8}$ & $F_{4}$ & $G_{2}$ \\
\hline Saturation factor & 36 & 144 & 3600 & 144 & 2,3 \\
\hline
\end{tabular}

Using these datas, one can easily check the computations of the $k_{s}$ given in the introduction reading the Dynkin diagrams. 


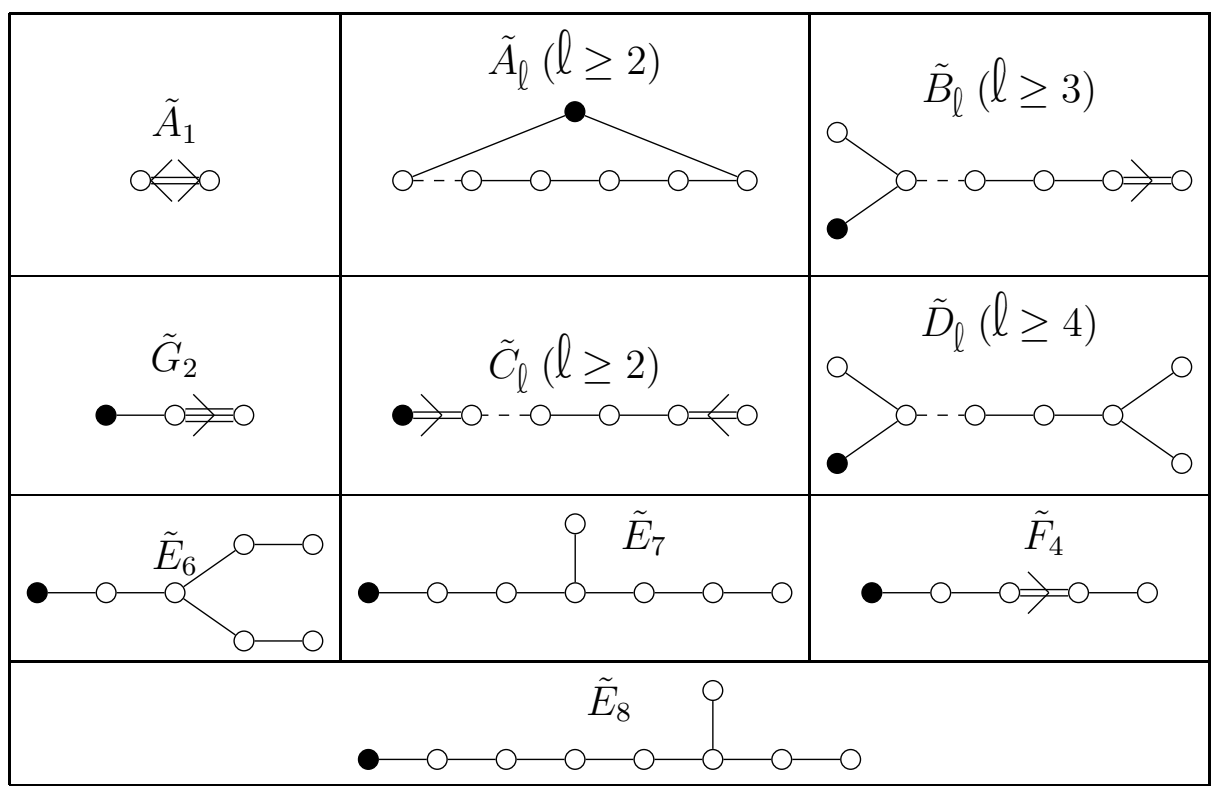

Proof of Theorems 3 and 4. Let $\left(\lambda_{1}, \lambda_{2}, \mu\right) \in\left(P_{+}\right)^{3}$ such that $\mu-\lambda_{1}-$ $\lambda_{2} \in Q$ and there exists $N>0$ such that $\left(N \lambda_{1}, N \lambda_{2}, N \mu\right) \in \Gamma_{\mathrm{N}}(\mathfrak{g})$. Up to tensoring with $L(\delta)$ one may assume that $\lambda_{1}(d)=\lambda_{2}(d)=0$. Write $\mu$ as $\bar{\mu}+n \delta$, with $n \in \mathrm{Z}$.

Set $b=\varphi\left(\lambda_{1}, \lambda_{2}, \bar{\nu}\right)$. By Proposition 4 , there exists $\left(u_{1}, u_{2}, v, i\right) \in \mathcal{I}$ such that $b=\varphi_{\left(u_{1}, u_{2}, v, i\right)}\left(\lambda_{1}, \lambda_{2}, \bar{\nu}\right)$.

We claim that $b k_{\mathfrak{g}}$ is an integer. The norm on $\dot{Q}^{\vee}$ is normalized by $\left\|\dot{\alpha}^{\vee}\right\|^{2}=2$ for a short coroot $\dot{\alpha}^{\vee} \in \dot{\Phi}^{\vee}$. Then, for any $h \in \dot{Q}^{\vee}$, we have $\frac{\|h\|^{2}}{2} \in \mathrm{Z}$. This can be proved easily by a case by case consideration. Then, formula (24) shows that $b \in \mathrm{Z}$ if $i=0$. If $i>0$, formula (25) shows that $k_{\mathfrak{g}} b \in \mathrm{Z}$.

Consider $\left(k_{\mathfrak{g}} \lambda_{1}, k_{\mathfrak{g}} \lambda_{2}, k_{\mathfrak{g}} \bar{\nu}+\left(k_{\mathfrak{g}} b\right) \delta\right)$. Observe the $Q=\dot{Q}+\mathrm{z} \delta$. Then, since $\nu-\lambda_{1}-\lambda_{2} \in Q, k_{\mathfrak{g}} \bar{\nu}+\left(k_{\mathfrak{g}} b\right) \delta-k_{\mathfrak{g}} \lambda_{1}-k_{\mathfrak{g}} \lambda_{2} \in Q$. For any $\lambda \in \mathfrak{h}_{\mathrm{Z}}^{*}$ and $w \in W, \lambda-w \lambda \in Q$. Hence, $v^{-1} k_{\mathfrak{g}} \bar{\nu}+\left(k_{\mathfrak{g}} b\right) \delta-u_{1}^{-1} k_{\mathfrak{g}} \lambda_{1}-u_{2}^{-1} k_{\mathfrak{g}} \lambda_{2}$ belongs to $Q$. Since $\left(N \lambda_{1}, N \lambda_{2}, N \nu\right) \in \Gamma_{\mathrm{N}}(\mathfrak{g})$, Corollary 1 implies that $\left(u_{1}^{-1} \lambda_{1}, u_{2}^{-1} \lambda_{2}, v^{-1}(\bar{\nu}+b \delta)\right)$ belongs to $\Gamma\left(L_{i}\right)$. But $k_{s}$ is a saturation factor for the group $L_{i}$. Then $\left(u_{1}^{-1} k_{s} k_{\mathfrak{g}} \lambda_{1}, u_{2}^{-1} k_{s} k_{\mathfrak{g}} \lambda_{2}, v^{-1} k_{s} k_{\mathfrak{g}}(\bar{\nu}+b \delta)\right)$ belongs to $\Gamma\left(L_{i}\right)$. Corollary 1 implies that $\left(k_{s} k_{\mathfrak{g}} \lambda_{1}, k_{s} k_{\mathfrak{g}} \lambda_{2}, k_{s} k_{\mathfrak{g}}(\bar{\nu}+b \delta)\right)$ belongs to $\Gamma_{\mathrm{N}}(\mathfrak{g})$.

Proposition 1 implies that $n \leq b$. Then $k_{\mathfrak{g}}(b-n) \in \mathrm{z}_{\geq 0}$.

If $b=n$, we already proved that $\left(k_{s} k_{\mathfrak{g}} \lambda_{1}, k_{s} k_{\mathfrak{g}} \lambda_{2}, k_{s} k_{\mathfrak{g}} \nu\right)$ belongs to $\Gamma_{\mathrm{N}}(\mathfrak{g})$. Theorem 3 is proved in this case.

Moreover, Proposition 1 implies that the integer $b_{0}$ of Lemma 26 for $\left(k_{s} k_{\mathfrak{g}} \lambda_{1}, k_{s} k_{\mathfrak{g}} \lambda_{2}, k_{s} k_{\mathfrak{g}} \bar{\nu}\right)$ is equal to $k_{s} k_{\mathfrak{g}} b$. Then, Lemma 26 implies that $\left(k_{s} k_{\mathfrak{g}} \lambda_{1}, k_{s} k_{\mathfrak{g}} \lambda_{2}, k_{s} k_{\mathfrak{g}} \bar{\nu}+m \delta\right) \in \Gamma_{\mathrm{N}}(\mathfrak{g})$, for any

$$
m \leq k_{s} k_{\mathfrak{g}} b-2
$$


Assume that $k_{\mathfrak{g}}(b-n) \in \mathrm{Z}_{>0}$. If $k_{s}>1, m=k_{s} k_{\mathfrak{g}} n$ satisfies condition (37). Similarly, for any $d>1, k_{s} k_{\mathfrak{g}} n-d$ satisfies condition (37). The theorems follow in these cases.

Assume now that $k_{s}=1$ and fix $d>1$. We may assume that $n \neq b$. Then, the integer $b_{0}$ of Lemma 26 for $\left(d k_{\mathfrak{g}} \lambda_{1}, d k_{\mathfrak{g}} \lambda_{2}, d k_{\mathfrak{g}} \bar{\nu}\right)$ is equal to $d k_{\mathfrak{g}} b$. Since $m=d k_{\mathfrak{g}} n=d\left(k_{\mathfrak{g}} n-k_{\mathfrak{g}} b\right)+d k_{\mathfrak{g}} n$ satisfies $m \leq d k_{\mathfrak{g}} b-2$, Theorem 3 also holds in this case.

\section{Some TECHNiCAL LEMmas}

In this section we collect some technical results on Birkhoff and Bruhat decompositions, on Geometric Invariant Theory...

11.1. Bruhat and Birkhoff decompositions. In this subsection, $G$ is the minimal Kac-Moody group associated to any symmetrizable GCM. Fix $T, W, B$ and $B^{-}$as usually. Let $P \supset B$ be a standard parabolic subgroup with standard Levi subgroup $L$. Fix a one parameter subgroup $\tau$ of $T$ such that for all $\beta \in \Phi, \beta \in \Phi(P)$ if and only if $\langle\beta, \tau\rangle \geq 0$.

Lemma 11.1. Let $u \in W$ and $v \in W^{P}$ such that $u \neq v$. Let $x \in$ $\stackrel{\circ}{X}_{G / B^{-}}^{u^{-1}}$.

Then $\lim _{t \rightarrow 0} \tau(t) x$ does not belong to $v^{-1} B \underline{O}^{-}$.

Proof. Recall that $\left(G / B^{-}\right)^{\tau}$ can be decomposed in the two following ways:

$$
\left(G / B^{-}\right)^{\tau}=\sqcup_{w \in W^{P}} L w^{-1} \underline{o}^{-}=\sqcup_{w \in W}(B \cap L) w \underline{o}^{-} .
$$

Moreover $\left\{w \underline{o}^{-}: w \in W\right\}$ are exactly the $T$-fixed points in $G / B^{-}$. Hence, if $Y \subset G / B^{-}$is $(B \cap L)$-stable then $Y^{\tau}=\sqcup_{x \in Y^{T}}(B \cap L) x$. For $Y=B u^{-1} \underline{o}^{-}$we get

$$
\left(B u^{-1} \underline{Q}^{-}\right)^{\tau}=(B \cap L) u^{-1} \underline{o}^{-} .
$$

If $v \in W^{P}$ then $\left(v^{-1} B v\right) \cap L=B \cap L$. Hence, for $Y=v^{-1} B \underline{o}^{-}$, we get

$$
\left(v^{-1} B \underline{o}^{-}\right)^{\tau}=(B \cap L) v^{-1} \underline{o}^{-} .
$$

Since $\lim _{t \rightarrow 0} \tau(t) x$ belongs to $\left(B u^{-1} \underline{Q}^{-}\right)^{\tau}$, we deduce that it does not belong to $v^{-1} B \underline{O}^{-}$.

Lemma 11.2. Let $u, v \in W$ such that $l(v)=l(u)+1$.

(i) Let $x_{1}, x_{2} \in \stackrel{\circ}{X}{ }_{G / B^{-}}^{u}$ such that $\lim _{t \rightarrow \infty} \tau(t) x_{1}=\lim _{t \rightarrow \infty} \tau(t) x_{2}$ belongs to $\stackrel{\circ}{X}_{G / B^{-}}^{v}$.

Then $\tau\left(\mathrm{C}^{*}\right) x_{1}=\tau\left(\mathrm{C}^{*}\right) x_{2}$.

(ii) Let $x_{1}, x_{2} \in \stackrel{\circ}{X}_{v}^{G / B}$ such that $\lim _{t \rightarrow \infty} \tau(t) x_{1}=\lim _{t \rightarrow \infty} \tau(t) x_{2}$ belongs to $\stackrel{\circ}{X}_{u}^{G / B}$.

Then $\tau\left(\mathrm{C}^{*}\right) x_{1}=\tau\left(\mathrm{C}^{*}\right) x_{2}$. 
Proof. Let us prove the first assertion. Set $y=\lim _{t \rightarrow \infty} \tau(t) x_{1}$. Then $y \in\left(\stackrel{\circ}{X}_{G / B^{-}}^{v}\right)^{\tau}=(B \cap L) v \underline{\underline{o}}^{-}$. Fix $l \in B \cap L$ such that $y=l v \underline{\underline{o}}^{-}$. Moreover, $P y=P x_{1}=P x_{2}$. Note that for any $g^{u} \in P^{u,-}, l^{\prime} \in L$ and $w \in W$, we have

$$
\lim _{t \rightarrow \infty} \tau(t) g^{u} l^{\prime} w \underline{O}^{-}=l^{\prime} w \underline{o}^{-} .
$$

One deduces that there exist $g_{i}^{u} \in P^{u,-}$ such that $x_{i}=g_{i}^{u} y$, for $i=1,2$. Then $l^{-1} x_{i}=\left(l^{-1} g_{i}^{u} l\right) v \underline{O}^{-}$belongs to $\dot{X}_{v}^{G / B^{-}}$. Since $l \in B, l^{-1} x_{i}$ also belongs to $\stackrel{\circ}{X}_{G / B^{-}}^{u}$. Finally, $l^{-1} x_{i}$ belongs to $\stackrel{\circ}{X}_{G / B^{-}}^{u} \cap \stackrel{\circ}{X}_{v}^{G / B^{-}}$.

But $l(v)=l(u)+1$ and $\stackrel{\circ}{X}_{G / B^{-}}^{u} \cap \stackrel{\circ}{X}_{v}^{G / B^{-}}$is isomorphic to $\mathrm{C}^{*}$. Since $l^{-1} x_{1}$ and $l^{-1} x_{2}$ are not fixed by $\tau\left(\mathrm{C}^{*}\right)$ they belong to the same $\tau$-orbit. Since the actions of $\tau$ and $L$ commute, one deduces that $\tau\left(\mathrm{C}^{*}\right) x_{1}=$ $\tau\left(\mathrm{C}^{*}\right) x_{2}$.

The second assertion works similarly. Up to translating by an element of $B \cap L$, one may assume that $\lim _{t \rightarrow \infty} \tau(t) x_{1}=\lim _{t \rightarrow \infty} \tau(t) x_{2}=$ uo. Then $x_{1}$ and $x_{2}$ belong to $\stackrel{\circ}{X}_{G / B}^{u} \cap \stackrel{\circ}{X}_{v}^{G / B}$ that is isomorphic to $\mathrm{C}^{*}$.

Lemma 11.3. Let $Q_{1}, Q_{2}$ be two parabolic subgroups of $G$ containing $B^{-}$. Consider $\mathrm{X}=G / Q_{1} \times G / Q_{2} \times G / P$ with base point $\left(\underline{o}_{1}, \underline{o}_{2}, \underline{o}\right)$. Let $P$ be a standard parabolic subgroup of finite type and $L$ denote its standard Levi subgroup. Fix $l \in L$. Let $u_{1}, u_{2}$, and $v$ in $W^{P}$. Set $\mathrm{x}_{0}=\left(l u_{1}^{-1} \underline{o}_{1}, u_{2}^{-1} \underline{o}_{2}, v^{-1} \underline{o}\right) \in \mathrm{X}$ and $\mathcal{O}=G \cdot \mathrm{x}_{0}$.

Then

$$
\left\{x \in \mathcal{O}: \lim _{t \rightarrow 0} \tau(t) x \in L . \mathrm{x}_{0}\right\}=P . \mathrm{x}_{0} .
$$

Proof. Consider first the anologous situation in $G / Q_{2} \times G / B$, with its two projections $p_{1}$ and $p_{2}$ on $G / Q_{2}$ and $G / B$. Set $\mathrm{x}_{1}=\left(u_{2}^{-1} \underline{Q}_{2}, v^{-1} \underline{o}\right)$, $\mathcal{O}_{1}=G$. $\mathrm{x}_{1}$ and $\mathcal{O}_{1}^{0}=L . \mathrm{x}_{1}$. Set also $\mathcal{O}_{1}^{+}=\left\{x \in \mathcal{O}_{1}: \lim _{t \rightarrow 0} \tau(t) x \in\right.$ $\left.L . \mathrm{x}_{1}\right\}$. Then $p_{1}\left(\mathcal{O}_{1}\right)=G / B^{-}$and $p_{1}\left(\mathcal{O}_{1}^{0}\right)=L u_{2}^{-1} \underline{o}_{2}$. Moreover,

$$
\left\{x \in G / Q_{2}: \lim _{t \rightarrow 0} \tau(t) x \in L . u_{2}^{-1} \underline{o}\right\}=P . u_{2}^{-1} \underline{o} .
$$

Since $\mathcal{O}_{1}^{+}$is stable by $P$, it follows that

$$
\mathcal{O}_{1}^{+}=P . \mathcal{I} \text { where } \mathcal{I}=\left(\left\{u_{2}^{-1} \underline{\underline{O}}_{2}\right\} \times G / B\right) \cap \mathcal{O}_{1}^{+} .
$$

Set $\mathrm{x}_{2}=v^{-1} \underline{\underline{o}}$. Then, $p_{2}(\mathcal{I})$ is the set of points $x \in\left(u_{2}^{-1} Q_{2} u_{2}\right) \cdot \mathrm{x}_{2}$ such that $\lim _{t \rightarrow 0} \tau(t) x \in\left(L \cap u_{2}^{-1} Q_{2} u_{2}\right) \mathrm{x}_{2}$. In particular $p_{2}(\mathcal{I})$ is contained in $P \mathrm{x}_{2}$. In particular the weights of $\tau$ on $T_{\mathrm{x}_{2}} p_{2}(\mathcal{I})$ are nonnegative. On the other hand they are contained in $T_{\mathrm{x}_{2}}\left(u_{2}^{-1} Q_{2} u_{2}\right) \mathrm{x}_{2}$. It follows that $T_{\mathrm{x}_{2}} p_{2}(\mathcal{I})$ is contained in $T_{\mathrm{x}_{2}}\left(P \cap u_{2}^{-1} Q_{2} u_{2}\right) \mathrm{x}_{2}$. Note that, since $P$ has finite type, $P \cap u_{2}^{-1} Q_{2} u_{2}$ is finite dimensional. Moreover, the dimension of $\mathcal{I}\left(\right.$ at $\left.x_{2}\right)$ is at most equal to $\operatorname{dim}\left(\left(P \cap u_{2}^{-1} Q_{2} u_{2}\right) x_{2}\right)$. It follows that 
$\left(P \cap u_{2}^{-1} Q_{2} u_{2}\right) \mathrm{x}_{2}$ is open in $p_{2}(\mathcal{I})$. Since $\left(P \cap u_{2}^{-1} Q_{2} u_{2}\right) \mathrm{x}_{2}$ contains $\left(L \cap u_{2}^{-1} Q_{2} u_{2}\right) \mathrm{x}_{2}$, we deduce that

$$
p_{2}(\mathcal{I})=\left(P \cap u_{2}^{-1} Q_{2} u_{2}\right) \mathrm{x}_{2},
$$

and

$$
\mathcal{O}_{1}^{+}=P . \mathrm{x}_{1}
$$

Consider now

$$
\pi_{1}: \mathrm{X} \longrightarrow G / Q_{2} \times G / B,\left(x_{1}, x_{2}, x_{3}\right) \longmapsto\left(x_{2}, x_{3}\right) .
$$

Set $\mathcal{O}^{+}=\left\{x \in \mathcal{O}: \lim _{t \rightarrow 0} \tau(t) x \in L \cdot x_{0}\right\}$. Equality (38) and the fact that $\mathcal{O}^{+}$is $P$-stable imply that

$$
\mathcal{O}^{+}=P\left(\left(G / Q_{1} \times\left\{\mathrm{x}_{1}\right\}\right) \cap \mathcal{O}^{+}\right) .
$$

Note that

$$
\begin{array}{ll}
\left(G / Q_{1} \times\left\{\mathrm{x}_{1}\right\}\right) \cap \mathcal{O}=\left(u_{2}^{-1} Q_{2} u_{2} \cap v^{-1} B v\right) . \mathrm{x}_{0} & \text { and } \\
\left(G / Q_{1} \times\left\{\mathrm{x}_{1}\right\}\right) \cap \mathcal{O}^{0}=\left(u_{2}^{-1} Q_{2} u_{2} \cap v^{-1} B v \cap L\right) . \mathrm{x}_{0} &
\end{array}
$$

Then, since $u_{2}^{-1} Q_{2} u_{2} \cap v^{-1} B v$ is finite dimensional, [Res10, Lemma 12] shows that

$$
\left(G / Q_{1} \times\left\{\mathrm{x}_{1}\right\}\right) \cap \mathcal{O}^{+}=\left(P \cap u_{2}^{-1} Q_{2} u_{2} \cap v^{-1} B v\right) . \mathrm{x}_{0} .
$$

With equality (39) this ends the proof of the lemma.

11.2. Affine root systems. In this subsection, we consider an untwisted affine root system and use the notations of Section 9.1, Recall in particular, that $\dot{\mathfrak{h}}_{\mathrm{R}}^{*}$ is endowed with $\dot{W}$-Euclidean norm $\|\cdot\|$ such that $\|\theta\|^{2}=2$.

Lemma 11.4. Consider an affine Weyl group $W=\dot{Q}^{\vee} \cdot \dot{W}$. Set $N=$ $\sharp \dot{\Phi}^{+}$.

There exists a positive real constant $K$ such that for any $h \in \dot{Q}^{\vee}$ and $\dot{w} \in \dot{W}$, we have

$$
K\|h\|-N \leq l(h \dot{w}) \leq N+\sqrt{2} N\|h\| .
$$

Proof. Set $w=h \dot{w}$. The length of $w$ is the cardinality of $w^{-1} \Phi^{+} \cap \Phi^{-}$. One can deduce (see e.g. [IM65]) that:

$$
l(h \dot{w})=\sum_{\dot{\alpha} \in \dot{\Phi}^{+}, \dot{w}^{-1} \dot{\alpha} \in \dot{\Phi}^{+}}|\langle h, \dot{\alpha}\rangle|+\sum_{\dot{\alpha} \in \dot{\Phi}^{+}, \dot{w}^{-1} \dot{\alpha} \in \dot{\Phi}^{-}}|\langle h, \dot{\alpha}\rangle-1| .
$$

The inequality on the right just follows from

$$
\begin{aligned}
& |\langle h, \dot{\alpha}\rangle-1| \leq|\langle h, \dot{\alpha}\rangle|+1 \\
& |\langle h, \dot{\alpha}\rangle| \leq\|h\|\|\dot{\alpha}\| \leq \sqrt{2}\|h\| .
\end{aligned}
$$

Moreover,

$$
\begin{aligned}
l(h \dot{w}) & \geq l(h)-l(\dot{w}) \\
& \geq \sum_{\dot{\alpha} \in \dot{\Phi}^{+}}|\langle h, \dot{\alpha}\rangle|-N .
\end{aligned}
$$


Since $\dot{\Phi}^{+}$spans $\dot{\mathfrak{h}}_{\mathrm{R}}^{*}$, the map $h \mapsto \sum_{\dot{\alpha} \in \dot{\Phi}^{+}}|\langle h, \dot{\alpha}\rangle|$ is a norm on the real vector space $\dot{\mathfrak{h}}_{\mathrm{R}}^{*}$. This norm is equivalent to $\|\cdot\|$, and there exists $K$ such that $K\|h\| \leq \sum_{\dot{\alpha} \in \dot{\Phi}^{+}}|\langle h, \dot{\alpha}\rangle|$. The lemma follows.

11.3. Jacobson-Morozov's theorem. Let $\mathfrak{g}$ be an untwisted affine Kac-Moody Lie algebra and $\mathfrak{p}$ be a standard parabolic subalgebra. Let $G$ be the minimal Kac-Moody group associated to $\mathfrak{g}$ and $P$ be the parabolic subgroup corresponding to $\mathfrak{p}$. Fix $\tau$ a one parameter subgroup of $T$ in $\oplus_{\alpha_{j} \notin \Delta(P)} \mathrm{Z}_{>0} \varpi_{\alpha_{j}^{\vee}}$. Consider the action of $\tau$ on $\mathfrak{g}$ and the corresponding weight space decompositions

$$
\mathfrak{g}=\oplus_{n \in Z} \mathfrak{g}_{n} \quad \mathfrak{p}=\oplus_{n \in \mathbf{Z}} \mathfrak{g}_{n} .
$$

In $\mathfrak{s} l_{2}(\mathrm{C})$, we denote by $(E, H, F)$ the standard triple

$$
E=\left(\begin{array}{ll}
0 & 1 \\
0 & 0
\end{array}\right) \quad H=\left(\begin{array}{cc}
1 & 0 \\
0 & -1
\end{array}\right) \quad F=\left(\begin{array}{ll}
0 & 0 \\
1 & 0
\end{array}\right)
$$

satisfying

$$
[E, F]=H \quad[H, E]=2 E \quad[H, F]=-2 F .
$$

Proposition 11.1. Fix $n \in \mathrm{Z}_{>0}$. Let $w \in W$. Let $\xi$ be a nonzero vector in $\mathfrak{g}_{n} \cap w \mathfrak{u}^{-} w^{-1}$.

Then there exists a morphism $\phi: \mathrm{SL}_{2}(\mathrm{C}) \longrightarrow G$ of ind-groups such that $T_{e} \phi(E)=\xi$.

Proof. Observe that $\mathfrak{g}_{n}$ is contained in $\mathfrak{u}$. Then $\xi \in \mathfrak{u} \cap w \mathfrak{u}^{-} w^{-1}$ and by [Kum02, Theorem 10.2.5], $\operatorname{ad} \xi \in \operatorname{End}(\mathfrak{g})$ is locally nilpotent.

Set $\mathcal{K}=\mathrm{C}((t))=\mathrm{C}\left[t^{-1}\right][[t]]$ and $\mathcal{R}=\mathrm{C}\left[t, t^{-1}\right] \subset \mathcal{K}$. Consider the Lie algebras $\dot{\mathfrak{g}} \otimes \mathcal{R}$ and $\dot{\mathfrak{g}} \otimes \mathcal{K}$. Recall that $\mathrm{C} d \oplus \dot{\mathfrak{g}} \otimes \mathcal{R}$ is a semi-direct product and that

$$
0 \longrightarrow \mathrm{C} c \longrightarrow \mathfrak{g} \longrightarrow \mathrm{C} d \oplus \dot{\mathfrak{g}} \otimes \mathcal{R} \longrightarrow 0
$$

is a central extension. Consider also the canonical C-linear embedding $\iota: \dot{\mathfrak{g}} \otimes \mathcal{R} \longrightarrow \mathfrak{g}$. It is not an homomorphism of Lie algebras.

Note that the one parameter subgroup $\tau$ is equal to $\dot{\tau}+m d$ for some one parameter subgroup $\dot{\tau}$ of $\dot{T}$ and some positive integer $m$. Then, $\tau$ acts on $\dot{\mathfrak{g}} \otimes \mathcal{R}$ and $\dot{\mathfrak{g}} \otimes \mathcal{K}$ by C-linear automorphisms. Consider the decomposition

$$
\dot{\mathfrak{g}} \otimes \mathcal{R}=\oplus_{k \in \mathrm{Z}}(\dot{\mathfrak{g}} \otimes \mathcal{R})_{k}
$$

in $\tau$-eigenspaces. Since each $(\dot{\mathfrak{g}} \otimes \mathcal{R})_{k}$ is finite dimensional and $m$ is positive, we have

$$
\dot{\mathfrak{g}} \otimes \mathcal{K}=\oplus_{k \in \mathrm{Z}_{<0}}(\dot{\mathfrak{g}} \otimes \mathcal{R})_{k} \oplus \prod_{k \in \mathrm{Z}_{\geq 0}}(\dot{\mathfrak{g}} \otimes \mathcal{R})_{k} .
$$

Observe that, for any nonzero $k \in \mathrm{z}, \mathfrak{g}_{k}=(\dot{\mathfrak{g}} \otimes \mathcal{R})_{k}$. In particular, $\xi$ belongs to $\dot{\mathfrak{g}} \otimes \mathcal{R}$. We denote by $\bar{\xi}$ (resp. $\tilde{\xi}$ ) the element $\xi$ considered as an element of the Lie algebra $\dot{\mathfrak{g}} \otimes \mathcal{R}$ (resp. $\dot{\mathfrak{g}} \otimes \mathcal{K})$. 
Since $\operatorname{ad} \xi$ is locally nilpotent and $\operatorname{ad} \tilde{\xi}$ is $\mathcal{K}$-linear, ad $\tilde{\xi}$ is nilpotent. Applying Jacobson-Morozov's theorem (see e.g. [Bou05, VIII$\S 11$ Proposition 2]) to the Lie algebra $\dot{\mathfrak{g}} \otimes \mathcal{K}$ over the field $\mathcal{K}$ of characteristic zero, we get an $\mathfrak{s} l_{2}$-triple $(X, H, Y)$ in $\dot{\mathfrak{g}} \otimes \mathcal{K}$ such that $X=\tilde{\xi}$.

Write $Y=\sum_{k \in \mathrm{z}} Y_{k}$ according to the decomposition (41). Since the Lie bracket is graded, we have in $\dot{\mathfrak{g}} \otimes \mathcal{K}$

$$
\left[X,\left[X, Y_{-n}\right]\right]=-2 X \text {. }
$$

Set $H_{0}=\left[X, Y_{-n}\right]$ and $\mathfrak{n}=\operatorname{Ker}(\operatorname{ad} \tilde{\xi})$. Since $X$ is homogeneous, $\mathfrak{n}$ decomposes as $\oplus_{k \in Z_{<0}} \mathfrak{n}_{k} \oplus \prod_{k \in Z_{>0}} \mathfrak{n}_{k}$, where $\mathfrak{n}_{k}=\mathfrak{n} \cap(\dot{\mathfrak{g}} \otimes \mathcal{K})_{k}$. Note that $\left[X, Y_{-n}\right]+2 Y_{-n}$ belongs to $\mathfrak{n}_{-n}$. By [Kos59, Corollary 3.4], $\operatorname{ad} H_{0}+$ $2 \operatorname{Id}_{\mathfrak{g} \otimes \mathcal{K}}$ is injective and stabilizes each $\mathfrak{n}_{n}$. Moreover, $\mathfrak{n}_{-n}$ is $\left(\operatorname{ad} H_{0}-\right.$ $\left.2 \mathrm{Id}_{\mathfrak{g} \otimes \mathcal{K}}\right)$-stable and finite dimensional as a complex vector space. Then there exists $Y^{\prime} \in \mathfrak{n}_{-n}$ such that

$$
\left[X, Y_{-n}\right]+2 Y_{-n}=\left[X, Y_{-n}^{\prime}\right]+2 Y_{-n}^{\prime} .
$$

Then, $\left(X, H_{0}, Y_{-n}-Y_{-n}^{\prime}\right)$ is an $\mathfrak{s} l_{2}$-triple contained in $(\dot{\mathfrak{g}} \otimes \mathcal{K})_{n} \times(\dot{\mathfrak{g}} \otimes$ $\mathcal{K})_{0} \times(\dot{\mathfrak{g}} \otimes \mathcal{K})_{-n}$. In particular, this $\mathfrak{s} l_{2}$-triple is contained in $\dot{\mathfrak{g}} \otimes \mathcal{R}$. Hence, we get an $\mathcal{R}$-linear Lie algebra homomorphism

$$
\phi: \mathfrak{s} l_{2}(\mathcal{R}) \longrightarrow \dot{\mathfrak{g}} \otimes \mathcal{R}
$$

such that $\phi(E)=\xi$. Since $\mathrm{SL}_{2}$ is simply connected and $\mathcal{R}$ contains $\mathrm{Q}$, $\mathrm{ABD}^{+} 66$, Exposé XXIV, Proposition 7.3.1] implies that there exists a morphism

$$
\Phi: \mathrm{SL}_{2} \longrightarrow \dot{G}
$$

of $\mathcal{R}$-group schemes with $\phi$ as differential map at the identity. In particular, we get a morphsm of ind-groups

$$
\bar{\Phi}: \mathrm{SL}_{2}(\mathrm{C}) \longrightarrow \dot{G} \otimes \mathcal{R}
$$

such that $T_{e} \bar{\Phi}(E)=\xi$.

Consider now the semidirect product $\mathrm{C}^{*} \ltimes \dot{G} \otimes \mathcal{R}$ associated to the derivation $d$, and the central extension

$$
\{1\} \longrightarrow \mathrm{C}^{*} \longrightarrow \mathrm{T} \stackrel{\pi}{\longrightarrow} \mathrm{C}^{*} \ltimes \dot{G}(\mathcal{R}) \longrightarrow\{1\}
$$

Then $\pi^{-1}\left(\bar{\Phi}\left(\mathrm{SL}_{2}(\mathrm{C})\right)\right)$ is a central extension of $(P) \mathrm{SL}_{2}(\mathrm{C})$. Hence, it is isomorphic to either $\mathrm{C}^{*} \times(P) \mathrm{SL}_{2}(\mathrm{C})$ or $\mathrm{GL}_{2}(\mathrm{C})$. In each case, $\bar{\Phi}$ can be lift to a morphism to $\pi^{-1}\left(\bar{\Phi}\left(\mathrm{SL}_{2}(\mathrm{C})\right)\right)$. This concludes the proof of the proposition.

11.4. Geometric Invariant Theory. For a given $C^{*}$-variety $X$ and a given integer $k$, we denote by $\mathrm{C}[X]^{(k)}$ the set of regular functions $f$ on $X$ such that $(t . f)(x)=f\left(t^{-1} x\right)=t^{k} f(x)$, for any $t \in \mathrm{C}^{*}$ and $x \in X$.

Lemma 11.5. Let $X$ be a normal affine $\mathrm{C}^{*}$-variety. Let $D$ be a $\mathrm{C}^{*}$ stable irreducible divisor and set $U=X-D$. We assume that 
(i) $\forall x \in U \quad \lim _{t \rightarrow 0} t x$ does not exist in $X$.

(ii) $\forall x_{1}, x_{2} \in U \quad \lim _{t \rightarrow \infty} t x_{1}=\lim _{t \rightarrow \infty} t x_{2} \in D \Longrightarrow \mathrm{C}^{*} x_{1}=$ $\mathrm{C}^{*} x_{2}$.

(iii) $\forall x \in D \quad \lim _{t \rightarrow 0} t x$ does exist in $D$.

(iv) $\forall y \in D^{\mathrm{C}^{*}} \quad \exists x \in U \quad \lim _{t \rightarrow \infty} t x=y$.

Then, for any nonnegative integer $k$, the restriction map induces an isomorphism $\mathrm{C}[X]^{(k)} \simeq \mathrm{C}[U]^{(k)}$.

Remark. In assumption $(i i), \lim _{t \rightarrow \infty} t x_{1}=\lim _{t \rightarrow \infty} t x_{2} \in D$ means that the limits exist and belong to $D$.

Proof. Set $\tilde{X}=X \times \mathrm{C}, \tilde{U}=U \times \mathrm{C}$ and $\tilde{D}=D \times \mathrm{C}$. We define an action of $\mathrm{C}^{*}$ on $\tilde{X}$ by $t .(x, z)=(t . x, t z)$ for any $t \in \mathrm{C}^{*}, x \in X$ and $z \in \mathrm{C}$. Observe that $\mathrm{C}[\tilde{X}]^{\mathrm{C}^{*}}=\oplus_{k \in \mathrm{N}} \mathrm{C}[X]^{(k)} z^{k}$ and $\mathrm{C}[\tilde{U}]^{\mathrm{C}^{*}}=\oplus_{k \in \mathrm{N}} \mathrm{C}[U]^{(k)} z^{k}$. Then, it is sufficient to prove that $\mathrm{C}[\tilde{X}]^{\mathrm{C}^{*}}=\mathrm{C}[\tilde{U}]^{\mathrm{C}^{*}}$.

But, one can easily check that $\tilde{X}$ satisfy all the assumptions of the lemma. As a consequence, it is sufficient to prove the lemma for $k=0$.

Consider the commutative diagram

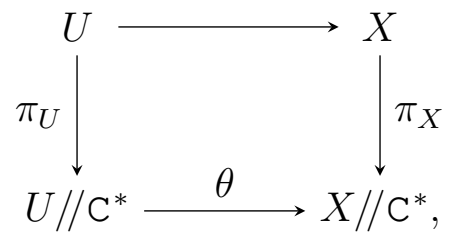

where $/ / \mathrm{C}^{*}$ denotes the GIT-quotient. It remains to prove that $\theta$ is an isomorphism.

We first prove the surjectivity of $\theta$. Let $\xi \in X / / \mathrm{C}^{*}$ and $\mathcal{O} \subset X$ be the unique closed $\mathrm{C}^{*}$-orbit in $\pi_{X}^{-1}(\xi)$. It $\mathcal{O} \subset U$, it is clear that $\theta\left(\pi_{U}(\mathcal{O})\right)=$ $\xi$. Otherwise $\mathcal{O} \subset D$. The orbit $\mathcal{O}$ being closed, assumption (iii) implies that $\mathcal{O}$ is a fixed point. By assumption (iv), there exists $\mathcal{O}^{\prime} \subset U$ such that $\overline{\mathcal{O}^{\prime}} \supset \mathcal{O}$. Then $\theta\left(\pi_{U}\left(\mathcal{O}^{\prime}\right)\right)=\xi$. We conclude that $\theta$ is surjective.

Let us prove now that $\theta$ is injective. Assume that $\xi_{1} \neq \xi_{2} \in U / / \mathrm{C}^{*}$ satisfy $\theta\left(\xi_{1}\right)=\theta\left(\xi_{2}\right)=$ : $\xi$. Let $\mathcal{O}_{1}, \mathcal{O}_{2} \subset U$ and $\mathcal{O} \subset X$ be the closed $\mathrm{C}^{*}$-orbit in $\pi_{U}^{-1}\left(\xi_{1}\right), \pi_{U}^{-1}\left(\xi_{2}\right)$ and $\pi_{X}^{-1}(\xi)$ respectively. Since $\pi_{X}\left(\mathcal{O}_{1}\right)=$ $\pi_{X}\left(\mathcal{O}_{2}\right)=\xi$, we have $\mathcal{O} \subset \overline{\mathcal{O}_{1}} \cap \overline{\mathcal{O}_{2}}$. In particular, $\mathcal{O}$ is a $\mathrm{C}^{*}$-fixed point and $\mathcal{O}_{1}$ and $\mathcal{O}_{2}$ are one dimensional. Pick $x_{1} \in \mathcal{O}_{1}, x_{2} \in \mathcal{O}_{2}$ and $y \in \mathcal{O}$. By assumption (ii), the limit $\lim _{t \rightarrow 0} t . x_{1}$ does not exist. But $y \in \overline{\mathcal{O}_{1}}-\mathcal{O}_{1}$, so $\lim _{t \rightarrow \infty} t . x_{1}=y$. Similarly $\lim _{t \rightarrow \infty} t . x_{2}=y$. Now, assumption (iii), implies that $\mathcal{O}_{1}=\mathcal{O}_{2}$. Hence $\theta$ is injective.

Since we work over complex numbers, the fact that $\theta$ is bijective implies that it is birational. By assumption $X$ is normal. Thus $X / / \mathrm{C}^{*}$ is normal. Then Zariski's main theorem (see e.g. Kum02, Theorem A.11]) implies that $\theta$ is an isomorphism. 


\section{REFERENCES}

$\left[\mathrm{ABD}^{+} 66\right]$ Michael Artin, Jean-Etienne Bertin, Michel Demazure, Alexander Grothendieck, Pierre Gabriel, Michel Raynaud, and Jean-Pierre Serre, Schémas en groupes, Séminaire de Géométrie Algébrique de l'Institut des Hautes Études Scientifiques, Institut des Hautes Études Scientifiques, Paris, 1963/1966.

[BB05] Anders Björner and Francesco Brenti, Combinatorics of Coxeter groups, Graduate Texts in Mathematics, vol. 231, Springer, New York, 2005.

[BK06] Prakash Belkale and Shrawan Kumar, Eigenvalue problem and a new product in cohomology of flag varieties, Invent. Math. 166 (2006), no. 1, 185228.

[BK10] - Eigencone, saturation and Horn problems for symplectic and odd orthogonal groups, J. Algebraic Geom. 19 (2010), no. 2, 199-242.

[BK14] Merrick Brown and Shrawan Kumar, A study of saturated tensor cone for symmetrizable Kac-Moody algebras, Math. Ann. 360 (2014), no. 3-4, 901-936.

[Bou05] Nicolas Bourbaki, Lie groups and Lie algebras. Chapters 7-9, Elements of Mathematics (Berlin), Springer-Verlag, Berlin, 2005, Translated from the 1975 and 1982 French originals by Andrew Pressley.

[DH98] Igor V. Dolgachev and Yi Hu, Variation of geometric invariant theory quotients, Inst. Hautes Études Sci. Publ. Math. 87 (1998), 5-56, With an appendix by Nicolas Ressayre.

[GKO85] P. Goddard, A. Kent, and D. Olive, Virasoro algebras and coset space models, Phys. Lett. B 152 (1985), no. 1-2, 88-92.

[IM65] N. Iwahori and H. Matsumoto, On some Bruhat decomposition and the structure of the Hecke rings of $\mathfrak{p}$-adic Chevalley groups, Inst. Hautes Études Sci. Publ. Math. (1965), no. 25, 5-48.

[Kam96] T. Kambayashi, Pro-affine algebras, Ind-affine groups and the Jacobian problem, J. Algebra 185 (1996), no. 2, 481-501.

[KKM09] Michael Kapovich, Shrawan Kumar, and John J. Millson, The eigencone and saturation for Spin(8), Pure Appl. Math. Q. 5 (2009), no. 2, Special Issue: In honor of Friedrich Herzebruch. Part 1, 755-780.

[KM06] Misha Kapovich and John J. Millson, Structure of the tensor product semigroup, Asian J. Math. 10 (2006), no. 3, 493-539.

[KM08] Michael Kapovich and John J. Millson, A path model for geodesics in Euclidean buildings and its applications to representation theory, Groups Geom. Dyn. 2 (2008), no. 3, 405-480.

[KN98] Shrawan Kumar and Madhav V. Nori, Positivity of the cup product in cohomology of flag varieties associated to Kac-Moody groups, Internat. Math. Res. Notices (1998), no. 14, 757-763.

[Kos59] Bertram Kostant, The principal three-dimensional subgroup and the Betti numbers of a complex simple Lie group, Amer. J. Math. 81 (1959), 9731032.

[KS09] Masaki Kashiwara and Mark Shimozono, Equivariant K-theory of affine flag manifolds and affine Grothendieck polynomials, Duke Math. J. 148 (2009), no. 3, 501-538.

[KT99] Allen Knutson and Terence Tao, The honeycomb model of $\mathrm{GL}_{n}(\mathbb{C})$ tensor products. I. Proof of the saturation conjecture, J. Amer. Math. Soc. 12 (1999), no. 4, 1055-1090.

[Kum02] Shrawan Kumar, Kac-Moody groups, their flag varieties and representation theory, Progress in Mathematics, vol. 204, Birkhäuser Boston Inc., Boston, MA, 2002. 
[Kum12] Shrawan Kumar, Positivity in T-Equivariant K-theory of flag varieties associated to Kac-Moody groups, ArXiv e-prints (2012).

[Kum14] Shrawan Kumar, A survey of the additive eigenvalue problem, Transform. Groups 19 (2014), no. 4, 1051-1148, With an appendix by M. Kapovich.

[Kum15] — Additive eigenvalue problem, Eur. Math. Soc. Newsl. (2015), no. 98, 20-27.

[KW88] Victor G. Kac and Minoru Wakimoto, Modular and conformal invariance constraints in representation theory of affine algebras, Adv. in Math. 70 (1988), no. 2, 156-236.

[MFK94] David Mumford, John Fogarty, and Frances Kirwan, Geometric invariant theory, 3d ed., Springer Verlag, New York, 1994.

[Pet04] Chris Peters, An introduction to complex algebraic geometry with emphasis on the theory of surfaces, Course notes available at https://www-fourier.ujf-grenoble.fr/ peters/ConfsAndSchools/surface.f/surfcourse.pd 2004.

[PR13] B. Pasquier and N. Ressayre, The saturation property for branching rules-examples, Exp. Math. 22 (2013), no. 3, 299-312.

[Res10] Nicolas Ressayre, Geometric invariant theory and generalized eigenvalue problem, Invent. Math. 180 (2010), 389-441.

[Res11] , Multiplicative formulas in Schubert calculus and quiver representation, Indag. Math. (N.S.) 22 (2011), no. 1-2, 87-102.

[Ric12] Edward Richmond, A multiplicative formula for structure constants in the cohomology of flag varieties, Michigan Math. J. 61 (2012), no. 1, 3-17.

[Sha81] I. R. Shafarevich, On some infinite-dimensional groups. II, Izv. Akad. Nauk SSSR Ser. Mat. 45 (1981), no. 1, 214-226, 240.

[Sta12] Immanuel Stampfli, On the topologies on ind-varieties and related irreducibility questions, J. Algebra 372 (2012), 531-541.

Institut Camille Jordan (ICJ), UMR CNRS 5208, Université Claude Bernard Lyon I, 43 Boulevard du 11 novembre 1918, F - 69622 VilleurBANNE CEDEX ressayre@math.univ-lyon1.fr 